\title{
Compactifications of ADE conformal matter on
}

\section{a torus}

\author{
Hee-Cheol Kim, ${ }^{a}$ Shlomo S. Razamat, ${ }^{b}$ Cumrun Vafa ${ }^{c}$ and Gabi Zafrir ${ }^{d}$ \\ ${ }^{a}$ Department of Physics, POSTECH, \\ Pohang 790-784, Korea \\ ${ }^{b}$ Department of Physics, Technion, \\ Haifa, 32000, Israel \\ c Jefferson Physical Laboratory, Harvard University, \\ Cambridge, MA 02138, U.S.A. \\ ${ }^{d}$ IPMU, University of Tokyo, \\ Kashiwa, Chiba 277-8583, Japan \\ E-mail: heecheol1@gmail.com, razamat@physics.technion.ac.il, \\ vafa@physics.harvard.edu, gabi.zafrir@ipmu.jp
}

ABSTRACT: In this paper we study compactifications of ADE type conformal matter, $N$ M5 branes probing $A D E$ singularity, on torus with flux for global symmetry. We systematically construct the four dimensional theories by first going to five dimensions and studying interfaces. We claim that certain interfaces can be associated with turning on flux in six dimensions. The interface models when compactified on a circle comprise building blocks for constructing four dimensional models associated to flux compactifications of six dimensional theories on a torus. The theories in four dimensions turn out to be quiver gauge theories and the construction implies many interesting cases of IR symmetry enhancements and dualities of such theories.

Keywords: Duality in Gauge Field Theories, Field Theories in Higher Dimensions, Supersymmetric Gauge Theory

ARXIV EPRINT: 1806.07620 


\section{Contents}

1 Introduction 1

2 Six dimensions 3

3 Five dimensions $\quad 6$

3.1 A-type domain walls $\quad 7$

$\begin{array}{lll}3.2 & \text { D-type domain walls } & 23\end{array}$

$\begin{array}{lll}3.3 & \text { E-type domain walls } & 31\end{array}$

4 Four dimensions $\quad 38$

4.1 Examples of $A \quad 41$

4.2 Examples of $D \quad 45$

4.3 Examples of $E \quad 54$

\section{Introduction}

Often one can construct conformal field theories as fixed point models of several different RG flows. RG flows might explicitly exhibit some of the properties of the fixed point CFT while other properties might only emerge in the deep IR. These explicitly exhibited properties can be very different depending on the flow.

A very rich plethora of examples of flows, terminating in interesting conformal field theories in four dimensions with some supersymmetry, is given by compactifications of $(1,0)$ theories on Riemann surfaces. The compactification depends first on the chosen $(1,0)$ model of which we have a wide but controlled variety of examples [1-3]. The CFTs inherit symmetry properties of the six dimensional model preserved by the details of the compactification. The details which can have an effect on the symmetry are the background gauge fields one can turn on. These involve holonomies and fluxes, with the latter giving a discrete set of different models while the former often parametrizing the conformal manifolds of the fixed point. In some cases the same CFT can be obtained as an IR description of a UV complete four dimensional asymptotically free theory. This description might exhibit the same symmetry properties as the flow starting with six dimensional model, or they can appear only in the IR. In this paper we discuss a huge variety of examples of such relations between six dimensional and four dimensional flows.

In particular we consider compactifications of $(G, \widetilde{G})$ conformal matter on a torus with flux for the global symmetry for the cases when $G$ is the same as $\widetilde{G}$. These models can be engineered as the low energy description of $M 5$ branes probing transverse $G$ type singularity of the corresponding ALE space. Such compactifications were considered before for various special instances of $G$. For example, $A_{0}[4-6], A[7-9], D[10,11]$, and $A D E$ on 

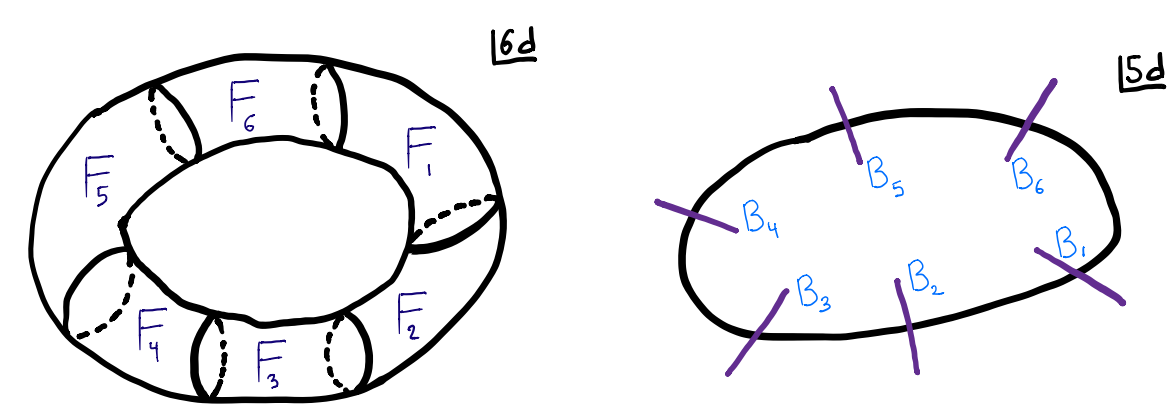

Figure 1. Compactification on a torus in six dimensions with flux $F$ for the global symmetry is constructed as a combination of blocks. Each block is associated with a tube and flux $F_{j}$ such that $\sum F_{j}=F$. The blocks are obtained by first going to five dimensions, considering then interfaces $B_{j}$, and then compactifying on an additional circle. This provides a systematic way to construct compactifications.

a torus with no flux [12-14]. Here we will perform a uniform analysis for all ADE cases with flux in the $G \times G$ symmetry by realizing that there is a natural way to get the models in four dimensions by first going through five dimensions. In five dimensions the theories are given by $G$ type affine quiver theories when the six dimensional models are put on a circle with proper choices of holonomies. We will argue that the flux for the global symmetry can be obtained in five dimensions as a sequence of duality interfaces relating affine quiver models with different mass parameters. The non obvious part of the statement is to find the description of the four dimensional theories living on the interfaces. In the cases relevant for us we will identify these as constructed from weakly coupled fields. Upon reduction to four dimensions we then will obtain theories having Lagrangians. These involve pairs of quiver theories in the shape of affine Dynkin diagrams with $\mathcal{N}=1$ matter content and where the links of the quiver are chiral bifundamentals. We will discover that there are certain choices which define the interface theory, which in turn determine the details of the chiral matter content of the theory. Altogether there are $2 \operatorname{Rank}(G)$ independent choices and they correspond to fluxes which we believe will cover arbitrary flux in the $G \times G$ global symmetry, as long as the flux is integral. ${ }^{1}$ We show that this is indeed the case in many examples. It would be interesting to clarify whether we get all possible fluxes in this way which we leave for future work. In the (A,A) case there is an additional $U(1)$ and we do not know how to construct interfaces corresponding to it. In fact the flux in the U(1) symmetry of class $\mathcal{S}$, that is $A_{0}$ compactification, do not have known weakly coupled Lagrangian, see for example $[9,15,16]$, so we expect naively this should be rather non-trivial in general.

We will engineer theories corresponding to compactification on torus with flux $F$ by combining together block theories to which we associate flux $F_{i}$ such that $\sum_{j} F_{j}=F$. See figure 1. The block theories will exhibit only abelian symmetries corresponding to the Cartan of the six dimensional model. For general values of flux this is also the expected

\footnotetext{
${ }^{1}$ By integral flux, we mean fluxes obeying the flux quantization condition. It is possible to also have fluxes that do not obey the quantization condition, which we shall refer to as fractional fluxes, if they are accompanied by additional elements compensating for it, see [10] for examples and details.
} 
symmetry of the theory compactified on the torus. However, for special values of flux the symmetry will contain non abelian factors. The typical situation for us is that we have a conformal manifold for the corresponding conformal theories each having (or arising from the IR limit of) weak coupling gauge theories, involving distinct quiver like theories. In some cases the dynamics of the gauge theories turns out to be rather interesting. For example, a way to view the quiver theories will be as a sequence of flows starting from weak coupled UV free theory flowing to IR which is strongly coupled, and then gauging additional global symmetries. The enhancement to the non abelian symmetry will emerge in this way of obtaining the models only at certain strongly coupled points. We will thus define a dictionary between four dimensional quiver theories and six dimensional compactifications. The check of this dictionary will involve anomaly computations and observation of the expected symmetry. Moreover, for the consistency of the considerations certain dualities should hold true. In some cases these are well known IR equivalences, while in other we will obtain novel types of dualities.

Let us here mention an important puzzle we do not resolve in this paper. Although our procedure passes all the tests for closed Riemann surfaces and tubes with integer flux, our basic minimal blocks, naively associated to tubes with fractional flux, do not pass the check of anomaly matching with six dimensional computation. There are two possible resolutions of this puzzle. One is that the minimal blocks do not correspond to tubes and only combining several of them such that the flux is integer corresponds to a tube. Second would be that there are subtleties with anomaly computation that we miss. We will define precisely our conjectures and leave this interesting puzzle for future work.

This paper is a third in a sequence following [10] and [11]. In the former we analyzed the case of $D_{4}$ minimal conformal matter, rank one E-string, on a torus and on general surfaces. The latter discussed minimal $D$ conformal matter on a torus but using a different five dimensional description than we do here. The different five dimensional descriptions lead to the interesting novel dualities we have mentioned.

The paper is organized as follows. In section 2 we discuss the six dimensional models and general issues of their reduction to five dimensions. In section 3 we discuss the six dimensional models on a circle. We will discuss the interface models and formulate the general conjecture of the relation of these to compactifications down on an additional circle. In section 4 we perform checks of the conjecture in four dimensions.

\section{Six dimensions}

We consider the $6 d$ SCFT living on $N$ M5-branes probing a transverse $\mathbb{C}^{2} / \Gamma$ singularity. Here $\Gamma$ is a discrete subgroup of $\mathrm{SU}(2)$, which is known to have an ADE classification. We shall use the notation $G$ for the ADE Lie group associated with $\Gamma$.

We next summarize some of the properties of these SCFTs that will be useful later. The most important property of the SCFTs that we need is their global symmetries. The Lie algebra of the global symmetry of these SCFTs is known to be $G \times G$, with the $A$ case having an extra $\mathrm{U}(1){ }^{2}$ To get a better understanding of both the global structure, and

\footnotetext{
${ }^{2}$ The symmetry is also enhanced in some special cases, as will become apparent from the low-energy gauge theory descriptions of these SCFTs that shall be discussed momentarily.
} 
the $4 d$ expectations from the compactification, we should also consider some elements of the operator spectrum of these theories.

For this it is useful to consider a different representation of these SCFTs. Besides the string theory construction, these theories can also be realized as UV completions of gauge or semi-gauge quiver theories, which can be employed to uncover some of their properties. In this description a special role is played by the $N=1$ cases, the so called minimal $(G, G)$ conformal matter [2]. The reason for that is that the generic $N$ cases can be built by taking $N$ minimal $(G, G)$ conformal matter theories and connecting them by gauging the symmetry $G$.

For example, take the $A_{k-1}$ case. Here the $N=1$ case is just a theory of $k^{2}$ free hypermultiplets, that can be grouped to form an $\mathrm{SU}(k) \times \mathrm{SU}(k)$ bifundamental. The $N=2$ case is then given by taking two such bifundamentals and connecting them by identifying and gauging an $\mathrm{SU}(k)$ group. This leads to the $6 d$ gauge theory $\mathrm{SU}(k)$ with $2 k$ fundamental hypermultiplets. For generic $N$ we have $N$ bifundamentals connected via $\mathrm{SU}(k)$ gauging, leading to the $6 d$ quiver gauge theory containing $N-1 \mathrm{SU}(k)$ gauge groups connected by bifundamental hypermultiplets, with $k$ fundamental hypermultiplets for each of the groups at the ends of the quiver.

In the $D_{k}$ case, the minimal conformal matter theory is a $\operatorname{USp}(2 k-8)$ gauge theory with $2 k$ hypermultiplets in the fundamental representation. Therefore, the general $N$ case is now an alternating $\mathrm{SO}-\mathrm{USp} 6 d$ quiver gauge theory. The low-energy description for the $E$ theories can also be constructed in this way, though the minimal conformal matter theories get progressively more involved. We refer the reader to [2] for a complete description of the low-energy theories for every $G$.

From the low-energy descriptions it is possible to read some of the operator spectrum of the SCFTs, where we shall concentrate on the feature shared for every $G$. First there are the moment map operators, which contain a scalar in the adjoint of $G \times G$ and in the 3 of $\mathrm{SU}(2)_{R}$, the R-symmetry of the theory. Additionally, all the SCFTs contain a bifundamental scalar operator in the $\left(\mathbf{F}_{G}, \mathbf{F}_{G}\right)$ of $G \times G$ where $\mathbf{F}_{G}$ is the fundamental representation of $G$. This operator transform in the $\mathbf{N p}_{\mathbf{G}}+\mathbf{1}$ dimensional representation of $\mathrm{SU}(2)_{R}$, where $p_{G}$ is a group dependent constant whose values for the various groups is given in table 1.

Besides these, there are various other operators which are group specific. For instance, in the $A$ case we naively have baryon operators. ${ }^{3}$ In the $D$ case, it is known that the minimal conformal matter theory possesses a non-perturbative state in the spinor of the SO group [11, 18], and it is thus expected to lead to bispinor states in the non-minimal case. While it may be interesting to gain a better understanding of the operator spectrum of these SCFTs, we shall not follow this further here.

One interesting observation that follows from our studies so far is that the global symmetry group of these SCFTs appear to be $\frac{G \times G}{Z_{G}}$. Here $Z_{G}$ stands for the center of $G$, and the modded group is the diagonal center of the two groups. For the readers convenience we have summarized these discrete groups for the relevant choices of $G$ in table 1 .

\footnotetext{
${ }^{3}$ For a study of the Higgs branch chiral ring operators in the $A$ type case, which are an interesting subset of the operators of the SCFT, see [17].
} 


\begin{tabular}{|c|c|c|c|c|c|}
\hline & $\mathrm{SU}(k)$ & $\mathrm{SO}(2 k)$ & $E_{6}$ & $E_{7}$ & $E_{8}$ \\
\hline$p_{G}$ & 1 & 2 & 4 & 6 & 12 \\
\hline$Z_{G}$ & $\mathbb{Z}_{k}$ & $\begin{array}{c}\mathbb{Z}_{2} \times \mathbb{Z}_{2}, k \text { even } \\
\mathbb{Z}_{4}, k \text { odd }\end{array}$ & $\mathbb{Z}_{3}$ & $\mathbb{Z}_{2}$ & 1 \\
\hline$|\Gamma|$ & $k$ & $4 k-8$ & 24 & 48 & 120 \\
\hline$r_{G}$ & $k-1$ & $k$ & 6 & 7 & 8 \\
\hline$d_{G}$ & $k^{2}-1$ & $k(2 k-1)$ & 78 & 133 & 248 \\
\hline$d_{F}$ & $\frac{1}{2}$ & 1 & 3 & 6 & 30 \\
\hline$t_{G}$ & $2 k$ & $2 k-8$ & 0 & 0 & 0 \\
\hline$u_{G}$ & 2 & 4 & 6 & 8 & 12 \\
\hline$h^{\vee}$ & $k$ & $2 k-2$ & 12 & 18 & 30 \\
\hline
\end{tabular}

Table 1. Various data used in this paper. Here $Z_{G}, r_{G}$, and $d_{G}$ are the center, rank and dimension of the group $G$ respectively. $|\Gamma|$ is the order of the finite group $\Gamma . d_{F}$ and $h^{\vee}$ are the Dynkin index of the fundamental representation and the dual Coxeter number of the group $G \cdot p_{G}, t_{G}$ and $u_{G}$ are various group dependent constants.

Anomalies from $6 \boldsymbol{d}$. We can estimate the anomalies of the $4 d$ theories resulting from the compactification of the $6 d$ theory, using the anomaly polynomial of the $6 d$ SCFT. For that we first need the expression for it, which was evaluated in [19]. The result can be written down for any group $G$ where it reads:

$$
\begin{aligned}
I= & \frac{1}{24}\left(|\Gamma|^{2} N^{3}-2 N\left(|\Gamma| r_{G}+|\Gamma|-1\right)+d_{G}-1\right) C_{2}^{2}(R) \\
& -\frac{1}{48}\left(N\left(|\Gamma| r_{G}+|\Gamma|-2\right)-d_{G}+1\right) p_{1}(T) C_{2}(R) \\
& -\frac{\left(|\Gamma| N-h^{\vee}\right)}{4 d_{F}} C_{2}(R)\left(C_{2}\left(G_{1}\right)_{\mathbf{F}}+C_{2}\left(G_{2}\right)_{\mathbf{F}}\right)+\frac{h^{\vee}}{48 d_{F}} p_{1}(T)\left(C_{2}\left(G_{1}\right)_{\mathbf{F}}+C_{2}\left(G_{2}\right)_{\mathbf{F}}\right) \\
& +\frac{\left(36 N u_{G}+d_{F}^{2} N t_{G}-3\right)}{24 N d_{F}^{2}}\left(C_{2}^{2}\left(G_{1}\right)_{\mathbf{F}}+C_{2}^{2}\left(G_{2}\right)_{\mathbf{F}}\right)-\frac{1}{4 N d_{F}^{2}} C_{2}\left(G_{1}\right)_{\mathbf{F}} C_{2}\left(G_{2}\right)_{\mathbf{F}} \\
& -\frac{t_{G}}{12}\left(C_{4}\left(G_{1}\right)_{\mathbf{F}}+C_{4}\left(G_{2}\right)_{\mathbf{F}}\right)+\frac{\left(30 N+7 d_{G}-23\right) p_{1}^{2}(T)-4\left(30 N+d_{G}-29\right) p_{2}(T)}{5760}
\end{aligned}
$$

Here $C_{2}(R)$ stands for the second Chern class in the fundamental representation of $\mathrm{SU}(2)_{R}$, and $p_{1}(T), p_{2}(T)$ stand for the first and second Pontryagin classes respectively. We also employ the notation $C_{n}(G)_{\mathbf{R}}$ for the n-th Chern class of the global symmetry $G$, evaluated in the representation $\mathbf{R}$ (here $F$ stands for fundamental). The rest of the symbols are various group theoretic constants whose values are given in table 1 .

Here we only write the anomalies for symmetries that appear generically. As previously mentioned, in the $A$ case there is an extra $\mathrm{U}(1)$ and the expression can be extended to include it. This case was studied extensively in [9], and we refer the reader there for more information. 
We next consider compactifying the theory on a torus and turning on non-trivial flux under various $\mathrm{U}(1)$ subgroups of the global symmetry $G \times G$. By integrating the anomaly polynomial 8-form of the $6 d$ theory on the Riemann surface we get the anomaly polynomial 6 -form of the resulting $4 d$ theory [5].

To do this we first need to decompose the various characteristic classes to those of the symmetries preserved in the presence of flux. First, the flux breaks half of the supersymmetry so that out of the original 8 supercharges only 4 remain. This corresponds to $\mathcal{N}=1$ in $4 d$. This also leads to the $\mathrm{SU}(2)_{R}$ symmetry of the $6 d$ theory being broken down to its $\mathrm{U}(1)$ Cartan, which becomes an R-symmetry in $4 d$. At the level of characteristic classes, these two are related by $C_{2}(R)=-C_{1}^{2}(R)$.

We also need to decompose the flavor symmetry characteristic classes to those of the symmetry preserved by the flux. In general, a symmetry $G$ is broken to $G \rightarrow\left(\prod \mathrm{U}(1)_{i}\right) \times$ $\left(\prod G_{a}^{\prime}\right)$, where $G_{a}^{\prime}$ are assumed to be non-abelian. In that case we can decompose:

$$
\begin{aligned}
C_{2}(G)_{\mathbf{F}}= & -2 \sum_{i, j} \xi_{i j} C_{1}\left(\mathrm{U}(1)_{i}\right) C_{1}\left(\mathrm{U}(1)_{j}\right)+\sum_{a} \mathfrak{i}_{a} C_{2}\left(G_{a}^{\prime}\right)_{\mathbf{F}} \\
C_{4}(G)_{\mathbf{F}}= & -2 \sum_{i, j, k, l} \lambda_{i j k l} C_{1}\left(\mathrm{U}(1)_{i}\right) C_{1}\left(\mathrm{U}(1)_{j}\right) C_{1}\left(\mathrm{U}(1)_{k}\right) C_{1}\left(\mathrm{U}(1)_{l}\right) \\
& +\sum_{i, j} \sum_{a} \tau_{i j}^{a} C_{1}\left(\mathrm{U}(1)_{i}\right) C_{1}\left(\mathrm{U}(1)_{j}\right) C_{2}\left(G_{a}^{\prime}\right)_{\mathbf{F}}+\sum_{i} \sum_{a} \rho_{i}^{a} C_{1}\left(\mathrm{U}(1)_{i}\right) C_{3}\left(G_{a}^{\prime}\right)_{\mathbf{F}}+\ldots,
\end{aligned}
$$

with the additional terms integrating to zero.

We next need to take the flux into account. This is done by setting $C_{1}\left(\mathrm{U}(1)_{i}\right)=$ $-z_{i} t+\epsilon_{i} C_{1}(R)+C_{1}\left(\mathrm{U}(1)_{i}^{4 d}\right)$, where $t$ is a unit 2 -form on the torus. The first term then takes the flux into account as $\int_{T^{2}} C_{1}\left(\mathrm{U}(1)_{i}^{4 d}\right)=-z_{i}$. The other terms then account for the $4 d$ curvature of the $\mathrm{U}(1)$, particularly the third term. The second term can be introduced to take account of the possible mixing of the $\mathrm{U}(1)$ with the R-symmetry. With this terms $C_{1}(R)$ measures the curvature of $\mathrm{U}(1)_{R}^{6 d}+\sum_{i} \epsilon_{i} \mathrm{U}(1)_{i}^{4 d}$. If one desires, the anomalies for the superconformal R-symmetry can be evaluated this way, with $\epsilon_{i}$ determined via amaximization.

All that remains is to evaluate the various constants appearing in the decomposition and perform the integration. We will not detail these computations as they are quite straightforward. In what follows we will only quote the result in various specific instances of various reductions from six dimensions. Reader interested in more details on the integration of anomaly polynomials from six to four dimensions can consult for example [5] and $[8,10]$.

\section{Five dimensions}

Let us consider $6 \mathrm{~d}(G, G)$ conformal matter theories compactified on a long cylinder. When the circle radius is small and with certain choices of holonomies for the global symmetries, the conformal matter theories reduce to affine ADE quiver gauge theories in 5d [2]. We can also consider flavor flux along the cylinder in $6 \mathrm{~d}$. As studied in $[10,11,20]$, the $6 \mathrm{~d}$ flux introduces interfaces, which we call flux domain walls, in the $5 \mathrm{~d}$ gauge theories. In this section, we propose Lagrangian constructions of these flux domain walls in the 5d quiver 
gauge theories. The five dimensional models then will be compactified to four dimensions leading to Lagrangians for torus or tube compactifications of the conformal matters.

\section{$3.1 \quad$ A-type domain walls}

We begin with flux domain walls in affine $A_{k-1}$ quiver gauge theories. For $N$ M5-branes, the $5 \mathrm{~d}$ theory is a circular quiver gauge theory consisting of $k \mathrm{SU}(N)$ gauge groups connected via bifundamental hypermultiplets of $\mathrm{SU}(N)_{i} \times \mathrm{SU}(N)_{i+1}$ symmetry (with $\mathrm{SU}(N)_{k+1}=$ $\left.\mathrm{SU}(N)_{1}\right)$. Classically, this theory has $\mathrm{U}(1)^{k}$ flavor symmetries of $k$ bifundamental hypermultiplets and $\mathrm{U}(1)^{k}$ topological instanton symmetries for the $k$ gauge nodes. We however expect that these classical abelian symmetries, when combined together, enhance in the $\mathrm{UV}$ to the $\mathrm{SU}(k)_{\beta} \times \mathrm{SU}(k)_{\gamma} \times \mathrm{U}(1)_{t}$ symmetry of the $6 \mathrm{~d}\left(\mathrm{SU}(k)_{\beta}, \mathrm{SU}(k)_{\gamma}\right)$ conformal matter theory by quantum instanton states. Here one U(1) global symmetry is identified with the Kaluza-Klein (KK) symmetry along the $6 \mathrm{~d}$ circle which will be ignored in what follows. In our notation, the $i$-th bifundamental hypermultiplet carries charges $\left(Q_{\beta_{i}}, Q_{\gamma_{i}}, t\right)=(1,-1,1)$ under the $\mathrm{U}(1)_{\beta_{i}} \times \mathrm{U}(1)_{\gamma_{i}} \times \mathrm{U}(1)_{t} \subset \mathrm{SU}(k)_{\beta} \times \mathrm{SU}(k)_{\gamma} \times \mathrm{U}(1)_{t}$ flavor symmetry.

Interfaces. Domain walls in $5 \mathrm{~d}$ theories can be constructed by joining two $5 \mathrm{~d}$ theories by a certain $4 \mathrm{~d}$ interface which is defined with boundary conditions of $5 \mathrm{~d}$ fields and their couplings to extra degrees of freedom living at the interface. Since the $6 \mathrm{~d}$ fluxes we are interested in preserve one half of the supersymmetries, the corresponding flux domain walls in $5 \mathrm{~d}$ must be $1 / 2$ BPS domain walls. We first suggest a type of $4 \mathrm{~d}$ interfaces which can consistently couple to $5 \mathrm{~d} 1 / 2$ BPS boundary conditions and then identify this domain wall configuration with the flux domain wall of the $6 \mathrm{~d}$ theory. The domain wall construction discussed in this subsection works also for other domain walls in the D- and E-type cases with minor changes.

The first step is to impose $1 / 2$ BPS boundary conditions at the interface $\left(x^{4}=0\right)$ for $5 \mathrm{~d}$ theories of the two chambers $x^{4}<0$ and $x^{4}>0$ respectively. We will choose Neumann boundary condition for the vector multiplets which sets the gauge fields at $x^{4}=0$ as

$$
\partial_{4} A_{\mu}=0(\mu=0,1,2,3), \quad A_{4}=0 .
$$

The $5 \mathrm{~d}$ vector multiplets with this boundary condition reduce to $4 \mathrm{~d} \mathcal{N}=1$ vector multiplets at $x^{4}=0$. Therefore, we have $G \times G^{\prime}$ gauge symmetries at the interface coming from the $5 \mathrm{~d}$ gauge fields in the left chamber (for $G=\mathrm{SU}(N)^{k}$ ) and in the right chamber (for $\left.G^{\prime}=\mathrm{SU}(N)^{\prime k}\right)$ respectively. For non-minimal D and E cases which we will discuss later, the gauge symmetry at the interface is a pair of two affine $D$ - and $E$-type quiver gauge symmetries, respectively. When $N=1$, on the other hand, the $\mathrm{SU}(1)$ gauge nodes in the affine quiver diagrams are replaced by two fundamental hypermultiplets for the adjacent gauge nodes.

For each bifundamental hypermultiplet with scalar fields $\Phi=(X, Y)$, we have two choices of boundary conditions:

$$
\text { 1) } \partial_{4} X=Y=0 \quad \text { or } \quad \text { 2) } \partial_{4} Y=X=0 \text {. }
$$


Under this 1/2 BPS boundary condition, a $5 \mathrm{~d}$ hypermultiplet reduces to a $4 \mathrm{~d} \mathcal{N}=1$ chiral multiplet at $x^{4}=0$ involving the scalar field, $X$ or $Y$, with Neumann boundary condition. We will denote the first boundary condition by + sign and the second boundary condition by - sign. So the boundary condition of $k$ bifundamental matters is labeled by a vector $\mathcal{B}=\left\{s_{1}, s_{2}, \cdots, s_{k}\right\}$ with $k$ signs $s_{i}= \pm$. Since there are two 5 d theories ending on the interface from both sides, we need a set of boundary conditions $\left(\mathcal{B}, \mathcal{B}^{\prime}\right)$ for the $5 \mathrm{~d}$ hypermultiplets in the first and the second chambers of the $5 \mathrm{~d}$ theory. For our domain walls, we shall impose the same boundary conditions $\mathcal{B}=\mathcal{B}^{\prime}$.

We now couple $4 \mathrm{~d}$ degrees of freedom at the interface to the $5 \mathrm{~d}$ boundary conditions. First, we introduce at the interface $4 \mathrm{~d}$ chiral multiplets $q_{i}$ in $(\overline{\mathbf{N}}, \mathbf{N})$ representation of $\mathrm{SU}(N)_{i} \times \mathrm{SU}(N)_{i}^{\prime}$ symmetry for $i=1,2, \cdots, k$. In addition, we add $4 \mathrm{~d}$ bifundamental chirals $\tilde{q}_{i}$ of $\mathrm{SU}(N)_{i+1} \times \mathrm{SU}(N)_{i}^{\prime}$ or $\mathrm{SU}(N)_{i} \times \mathrm{SU}(N)_{i+1}^{\prime}$ coupled to the other fields by the cubic superpotential of the forms

$$
\mathcal{W}_{x^{4}=0}=\sum_{i=+}\left(\tilde{q}_{i} q_{i} X_{i}+q_{i+1} \tilde{q}_{i} X_{i}^{\prime}\right)+\sum_{i=-}\left(Y_{i} \tilde{q}_{i} q_{i+1}+Y_{i}^{\prime} q_{i} \tilde{q}_{i}\right)
$$

where $X_{i}, Y_{j}$ and $X_{i}^{\prime}, Y_{j}^{\prime}$ stand for the $4 \mathrm{~d}$ chiral multiplets involving $5 \mathrm{~d}$ bifundamental scalars with Neumann boundary condition in the first and the second chambers, respectively. This superpotential equates the boundary conditions on two sides, i.e. $\mathcal{B}=\mathcal{B}^{\prime}$, as expected. Lastly, we add flip chiral fields coupled to the baryonic operators of the $4 \mathrm{~d}$ chirals $q_{i}$. We wish to remind the reader that the flip field $\phi_{\mathfrak{O}}$ for the operator $\mathfrak{O}$ is a gauge singlet chiral field which couples to $\mathfrak{O}$ through a superpotential, $\delta W=\phi_{\mathfrak{O}} \mathfrak{O}$. The effect of adding such a field is that the operator $\mathfrak{O}$ is set to zero in the chiral ring. Note also that the charges under flavor symmetries of $\phi_{\mathfrak{O}}$ are opposite to those of $\mathfrak{O}$ and that is the reason for the name flip field.

We can also consider similar domain walls by replacing the representations of $4 \mathrm{~d}$ chiral fields $q_{i}$ by $(\mathbf{N}, \overline{\mathbf{N}})$ and by coupling $4 \mathrm{~d}$ fields $q_{i}$ and $\tilde{q}_{i}$ to the $5 \mathrm{~d}$ boundary conditions through the superpotential of the form (3.3) accordingly. We remark here that these two choices of $4 \mathrm{~d}$ fields $q_{i}$ in either $(\overline{\mathbf{N}}, \mathbf{N})$ or $(\mathbf{N}, \overline{\mathbf{N}})$ lead to two different types of domain walls: the former gives domain walls for the flux on $\mathrm{SU}(k)_{\beta}$, and the latter leads to domain walls for the flux on $\mathrm{SU}(k)_{\gamma}$. We will distinguish these two types of domain walls by the subscript $\mathcal{T}=\beta$ or $\gamma$. We will first discuss the domain walls for $\mathcal{T}=\beta$ with $q_{i}$ in $(\overline{\mathbf{N}}, \mathbf{N})$ and then discuss the domain walls for $\mathcal{T}=\gamma$ with $q_{i}$ in $(\mathbf{N}, \overline{\mathbf{N}})$ later.

Figure 2 depicts two domain wall examples with boundary conditions $\mathcal{B}=\{+,+,+,-,-\}$ and $\mathcal{B}=\{+,-,+,-,-\}$ in the $A_{4}$ quiver gauge theory. There are cubic superpotentials of the form (3.3) for the triangles in the quiver diagrams. The boxes in the quiver diagrams represent the $G \times G^{\prime}$ symmetries at the interface and these symmetries will be gauged by the 5 d vector multiplets with Neumann boundary condition in two chambers. ${ }^{4}$

The boundary conditions and the $4 \mathrm{~d}$ couplings at the interface define a domain wall in the $5 \mathrm{~d}$ gauge theory. Let us now check if this domain wall is consistent with the $5 \mathrm{~d}$

\footnotetext{
${ }^{4}$ We shall generically use boxes for $4 \mathrm{~d}$ global symmetries and circles for gauge symmetries. When discussing interfaces in $5 \mathrm{~d}$ we use boxes for symmetries gauged by $5 \mathrm{~d}$ vector multiplets as, later when we discuss the reduction to $4 \mathrm{~d}$ on intervals, these become $4 \mathrm{~d}$ global symmetries.
} 

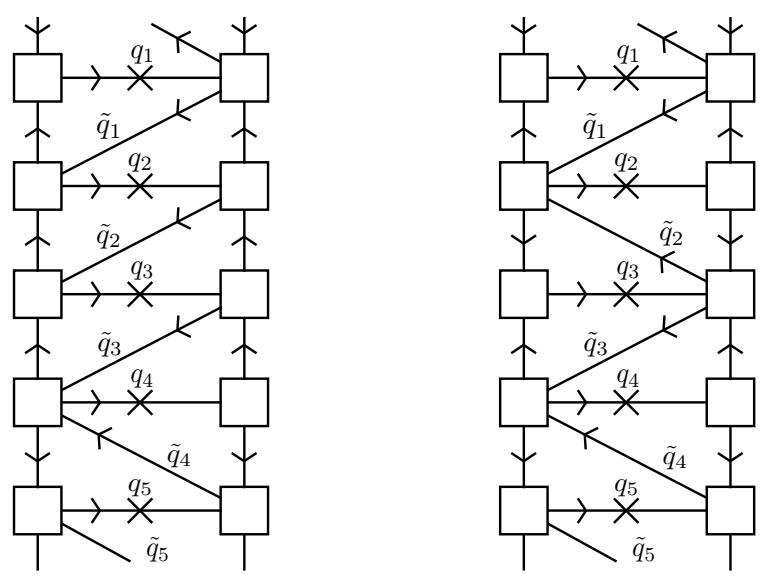

Figure 2. Quiver diagrams for the domain walls in the $A_{4}$ quiver gauge theory. The domain wall on the left is for the boundary condition $\mathcal{B}=\{+,+,+,-,-\}$ and the domain wall on the right is for $\mathcal{B}=\{+,-,+,-,-\}$. The square boxes denote the $\mathrm{SU}(N)^{5} \times \mathrm{SU}(N)^{\prime 5}$ gauge symmetries of $5 \mathrm{~d}$ gauge theories on both sides of the walls. The symbol $\times$ denotes flip fields coupled to the baryonic operator made from $q_{i}$.

gauge theory. The boundary conditions of the $5 \mathrm{~d}$ bulk fields induce non-trivial $4 \mathrm{~d}$ gauge anomalies at the interface. For being a consistent domain wall, these gauge anomalies must be canceled by the extra $4 \mathrm{~d}$ fields living on the boundary.

Let us first discuss cubic anomalies of the $\mathrm{SU}(N)^{k}$ gauge symmetries. The $i$-th hypermultiplet with boundary condition $s_{i}= \pm$ leaves a bifundamental chiral multiplet of $\mathrm{SU}(N)_{i} \times \mathrm{SU}(N)_{i+1}$ gauge symmetry at the boundary. This chiral multiplet leads to cubic gauge anomalies of the $\mathrm{SU}(N)_{i}$ and $\mathrm{SU}(N)_{i+1}$ symmetries given by

$$
\operatorname{Tr}\left(\mathrm{SU}(N)_{i}^{3}\right)=\frac{s_{i} N}{2}, \quad \operatorname{Tr}\left(\mathrm{SU}(N)_{i+1}^{3}\right)=-\frac{s_{i} N}{2} .
$$

Remember that we always need to multiply by the factor $\frac{1}{2}$ to all anomaly contributions from the $5 \mathrm{~d}$ hypermultiplets at boundaries [21-23]. This comes from the fact that the anomaly contributions of a chiral multiplet coming from the $5 \mathrm{~d}$ boundary condition equals one half of those from a $4 \mathrm{~d}$ chiral multiplet with the same charges.

We also need to take into account the anomalies from the $4 \mathrm{~d}$ bifundamental chiral multiplets $q_{i}$ and $\tilde{q}_{j}$. The $4 \mathrm{~d}$ chiral field $q_{i}$ contributes to the $\mathrm{SU}(N)_{i}$ anomaly as $\operatorname{Tr}\left(\mathrm{SU}(N)_{i}^{3}\right)=-N$. Another chiral field $\tilde{q}_{i}$ has cubic gauge anomaly $\operatorname{Tr}\left(\mathrm{SU}(N)_{i+1}\right)=N$ for $s_{i}=+$ and $\operatorname{Tr}\left(\mathrm{SU}(N)_{i}\right)=N$ for $s_{i}=-$. One can easily see that the total cubic gauge anomalies in the domain wall vanish when we sum over all anomaly contributions from the boundary conditions and the $4 \mathrm{~d}$ chiral multiplets. The cubic gauge anomalies of $\mathrm{SU}(N)_{i}^{\prime}$ in the other chamber are canceled in the same way.

We then move on to the gauge-global mixed anomalies at the interface. Firstly, there are anomaly inflow contributions from the $5 \mathrm{~d}$ bulk gauge theory. The boundary condition of the $i$-th bifundamental hypermultiplet with $s_{i}$ in the first chamber induces the following 
anomalies at the boundary

$$
\begin{gathered}
\operatorname{Tr}\left(\mathrm{U}(1)_{t} \mathrm{SU}(N)_{i}^{2}\right)=\operatorname{Tr}\left(\mathrm{U}(1)_{t} \mathrm{SU}(N)_{i+1}^{2}\right)=\frac{s_{i} N}{4}, \\
\operatorname{Tr}\left(\mathrm{U}(1)_{\beta_{i}} \mathrm{SU}(N)_{i}^{2}\right)=\operatorname{Tr}\left(\mathrm{U}(1)_{\beta_{i}} \mathrm{SU}(N)_{i+1}^{2}\right)=\frac{s_{i} N}{4}, \\
\operatorname{Tr}\left(\mathrm{U}(1)_{\gamma_{i}} \mathrm{SU}(N)_{i}^{2}\right)=\operatorname{Tr}\left(\mathrm{U}(1)_{\gamma_{i}} \mathrm{SU}(N)_{i+1}^{2}\right)=-\frac{s_{i} N}{4},
\end{gathered}
$$

with $\sum_{i} \mathrm{U}(1)_{\beta_{i}}=\sum_{i} \mathrm{U}(1)_{\gamma_{i}}=0$. Also, the $5 \mathrm{~d} \mathrm{SU}(N)_{i}$ vector multiplet with Neumann boundary condition leads to the anomaly inflow contributions toward the $4 \mathrm{~d}$ boundary as

$$
\operatorname{Tr}\left(\mathrm{U}(1)_{R} \mathrm{SU}(N)_{i}^{2}\right)=\frac{N}{2},
$$

where $\mathrm{U}(1)_{R} \subset \mathrm{SU}(2)_{R}$.

In addition, there are anomaly inflows from the gauge kinetic terms $\frac{4 \pi^{2}}{g_{i}^{2}} \operatorname{Tr}\left(F_{i}^{2}\right)$. These terms can be considered as the $5 \mathrm{~d} \mathcal{N}=1$ mixed Chern-Simons terms between the $\mathrm{U}(1)_{I_{i}}$ instanton symmetry and the $\mathrm{SU}(N)_{i}$ gauge symmetry with background scalar field $\frac{4 \pi^{2}}{g_{i}^{2}}$ in the $\mathrm{U}(1)_{I_{i}}$ vector multiplet. In the presence of the $4 \mathrm{~d}$ boundary, these CS-terms generate anomaly inflows toward the boundary.

It should be noted that the contribution of this term is novel in this construction, and did not appear in previous discussions of $5 \mathrm{~d}$ domain walls in relation to the compactifications of $6 \mathrm{~d}$ SCFTs to $4 \mathrm{~d}$, like in $[10,11]$. The distinguishing feature in the cases discussed here is that the $5 \mathrm{~d}$ gauge theories contain more then one gauge group. Generically the topological symmetries of the $5 \mathrm{~d}$ gauge theory, together with the flavor symmetry, appear to form an affine version of the global symmetry of the SCFT, where the affine extension being associated with the Kaluza-Klein tower of the $5 \mathrm{~d}$ conserved current, which is expected to build the $6 \mathrm{~d}$ one. Therefore, these contain one additional $\mathrm{U}(1)$ which does not survive the $4 \mathrm{~d}$ reduction. In cases with a single gauge group in $5 \mathrm{~d}$, the topological $\mathrm{U}(1)$ is usually related to this symmetry, and so the contribution of the gauge kinetic term is unneeded as we are only concerned with anomalies of $4 \mathrm{~d}$ symmetries after the $4 \mathrm{~d}$ reduction. However, in the cases we consider here, the $5 \mathrm{~d}$ gauge theory has many gauge groups, and their topological symmetry should be related to symmetries appearing in $4 \mathrm{~d}$, with the exception of one combination. Therefore, the $5 \mathrm{~d}$ gauge kinetic terms should contribute to the anomalies of the $4 \mathrm{~d}$ theories and must be taken into account. In fact, the $4 \mathrm{~d}$ chiral fields $q_{i}$ and $\tilde{q}_{i}$ also carry the charges of this Kaluza-Klein symmetry and these charges are uniquely fixed by the gauge-global mixed anomaly cancellation and cubic superpotential terms. We will however ignore these charges as we are interested only in $4 \mathrm{~d}$ symmetries.

The instanton number $I_{i}$ and the baryon symmetry $B_{i}$ for the $i$-th gauge node are related to the Cartan generators $H_{i, \pm}$ of the enhanced $\mathrm{SU}(k) \times \mathrm{SU}(k)$ symmetry as $[24,25]$

$$
H_{i, \pm}=\frac{1}{4} \sum_{j} A_{i j} I_{j} \pm \frac{B_{i}}{2 N}
$$


where $A_{i j}$ is the Cartan matrix of $A_{k-1}$ symmetry. The mass parameters $m_{i, \pm}$ for the Cartans $H_{i, \pm}$ are associated to the gauge couplings $g_{i}$ and the mass parameters $m_{B, i}$ for $B_{i}$ as

$$
\frac{8 \pi^{2}}{g_{i}^{2}}=\frac{1}{2} \sum_{i} A_{i j}\left(m_{j,+}+m_{j,-}\right), \quad m_{B, i}=\frac{m_{i,+}-m_{i,-}}{N_{i}},
$$

where $N_{i}$ is $h_{i}^{\vee}$ for $i$-th gauge node. This implies that the kinetic term for the $\operatorname{SU}(N)_{i}$ symmetry induces the $4 \mathrm{~d}$ anomaly inflows as

$$
\begin{gathered}
\operatorname{Tr}\left(\mathrm{U}(1)_{\beta_{i}} \mathrm{SU}(N)_{i}^{2}\right)=\operatorname{Tr}\left(\mathrm{U}(1)_{\gamma_{i}} \mathrm{SU}(N)_{i}^{2}\right)=\frac{N}{4}, \\
\operatorname{Tr}\left(\mathrm{U}(1)_{\beta_{i}} \mathrm{SU}(N)_{i+1}^{2}\right)=\operatorname{Tr}\left(\mathrm{U}(1)_{\gamma_{i}} \mathrm{SU}(N)_{i+1}^{2}\right)=-\frac{N}{4} .
\end{gathered}
$$

We have similar anomaly inflow contributions for the $\mathrm{SU}(N)_{i}^{\prime}$ gauge symmetries from the $5 \mathrm{~d}$ boundary conditions in the other chamber.

The bulk contributions to the gauge-global mixed anomalies are not canceled by themselves, so the $\mathrm{U}(1)_{\beta_{i}}$ and $\mathrm{U}(1)_{\gamma_{i}}$ symmetries will be broken unless these anomalies are canceled by those from the $4 \mathrm{~d}$ fields at the interface. It turns out that all the $\mathrm{U}(1)$ flavor symmetry charges of the $4 \mathrm{~d}$ chiral multiplets at the interface are uniquely fixed by requiring that all the Cartans of $\mathrm{SU}(k)_{\beta} \times \mathrm{SU}(k)_{\gamma} \times \mathrm{U}(1)_{t}$ are gauge anomaly free, and that there are no additional flavor symmetries together with the superpotential constraints, with the exception of the two cases with the most symmetric boundary conditions, i.e. $s_{i}=+$ or $s_{i}=-$ for all $i$ 's. We demand this property for the domain walls realizing the $6 \mathrm{~d}$ flux because the $6 \mathrm{~d}$ flux compactified on a circle breaks no Cartans of the flavor symmetry. Under this requirement, for example, $\mathrm{U}(1)_{R}$ charges for the $4 \mathrm{~d}$ chiral multiplets $q_{i}$ and $\tilde{q}_{i}$ are fixed to be 0 and +1 respectively. Two examples of domain walls in the $A_{4}$ quiver theory are presented in figure 3 . Here the $\mathrm{U}(1)$ charges of the $4 \mathrm{~d}$ fields, which are determined by this requirement, are denoted by the $\mathrm{U}(1)_{\beta}^{k-1} \times \mathrm{U}(1)_{\gamma}^{k-1} \times \mathrm{U}(1)_{t}$ fugacities.

On the other hand, when $\mathcal{B}=(+,+,+, \cdots,+)$ or $\mathcal{B}=(-,-,-, \cdots,-)$ (so when $\mathcal{B}$ is the most symmetric), we find that there exists an additional $\mathrm{U}(1)$ global symmetry apart from the bulk symmetry which does not arise from the circle reduction of the $6 \mathrm{~d}$ theory with flux. Thus, we lose an interpretation for the most symmetric cases as a compactification of the six dimensional theory with flux. So we will not discuss the most symmetric boundary conditions from now on, however see the next section for a possible roundabout interpretation in four dimensions.

The relation between four and six dimensions. We have constructed consistent domain wall configurations for $5 \mathrm{~d}$ boundary condition $\mathcal{B}$ 's. Let us now relate these domain walls in the $5 \mathrm{~d}$ gauge theory to the $6 \mathrm{~d}$ theory compactified on a $2 \mathrm{~d}$ surface with flux.

We note that this domain wall permutes the $\mathrm{U}(1)$ global symmetries of the $5 \mathrm{~d}$ theory. More precisely, when we pass through it, the $\mathrm{U}(1)_{\beta_{i}}$ symmetries acting on the hypermultiplets with the ' + ' boundary condition are cyclically permuted among themselves, and similarly the $\mathrm{U}(1)_{\beta_{j}}$ symmetries on the hypermultiplets with '-' boundary condition are permuted. We will label such permutations for $\mathrm{U}(1)_{\beta}^{k-1}$ and $\mathrm{U}(1)_{\gamma}^{k-1}$ by $\sigma_{\beta}$ and $\sigma_{\gamma}$ respectively. For a given $\mathcal{B}$, the $\sigma_{\beta}(\mathcal{B})$ is defined as a clockwise permutation of $\mathrm{U}(1)_{\beta_{i}}$ symmetries 

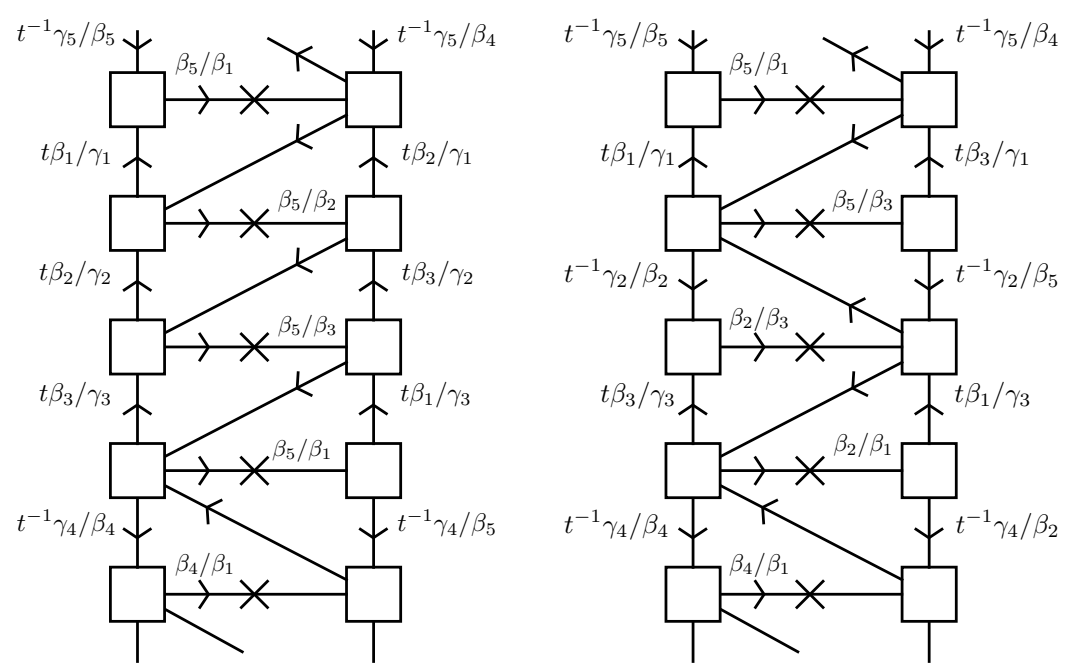

Figure 3. Domain walls for $\mathcal{B}=\{+,+,+,-,-\}$ (left) and $\mathcal{B}=\{+,-,+,-,-\}$ (right). The $\mathrm{U}(1)$ global charges of the $4 \mathrm{~d}$ chiral fields denoted by their fugacities are fixed by the gauge-global mixed anomaly cancellation and superpotential terms. Here the permutation $\sigma_{\beta}=\left(\begin{array}{llll}1 & 2 & 3\end{array}\right)\left(\begin{array}{ll}4 & 5\end{array}\right)$ and $\sigma_{\gamma}=\emptyset$ for the left tube. For the right tube $\sigma_{\beta}=\left(\begin{array}{ll}13 & 3\end{array}\right)(254)$ amd $\sigma_{\gamma}=\emptyset$.

with $s_{i}=+$ and a counterclockwise permutation of $\mathrm{U}(1)_{\beta_{j}}$ symmetries with $s_{j}=-$. The permutation $\sigma_{\gamma}(\mathcal{B})$ is trivial for the above domain walls involving the $4 \mathrm{~d}$ chiral fields $q_{i}$ with representations associated with the choice $\mathcal{T}=\beta$. As we will propose soon, these domain walls are associated to $\mathrm{SU}(k)_{\beta}$ flux in $6 \mathrm{~d}$. We will construct another type of domain walls with non-trivial $\sigma_{\gamma}(\mathcal{B})$ below which come with 4 d fields $q_{i}$ of other type with $\mathcal{T}=\gamma$. Note however that the permutations will not specify the domain wall model in a unique way. This is because the permutations are invariant under cyclic permutations of + and - , whereas the corresponding interface theories are different.

The definition of the permutations coming with the interfaces theory suffices for us to make the basic statement about relation of the interface models and compactifications to four dimensions. We conjecture the following:

\section{Conjectures}

1. A flux domain wall with total flux $F_{\text {tot }}$ in the $5 \mathrm{~d}$ affine $G$ quiver theory on a circle realizes the $6 \mathrm{~d}(G, G)$ conformal matter theory with flux $F_{\text {tot }}$ on a torus.

2. When $\prod_{i=1}^{l} \sigma^{t_{i}}=1$, the flux domain wall with total flux $F_{\text {tot }}$ in the $5 \mathrm{~d}$ affine $G$ quiver theory on an interval realizes the $6 \mathrm{~d}(G, G)$ conformal matter theory with flux $F_{\text {tot }}$ on a cylinder.

Here, $G=A_{k}$ for A-type domain walls and we defined $\sigma^{t_{i}}=\left(\sigma_{\beta}\left(\mathcal{B}_{i}\right), \sigma_{\gamma}\left(\mathcal{B}_{i}\right)\right)$ for $i$-th domain wall with boundary condition $\mathcal{B}_{i}$. We will propose the same conjectures also with $G=D_{k}, E_{k}$ for D-type and E-type flux domain walls which will be discussed below in detail.

The flux of the single domain wall, which we will call basic domain wall, is to be computed soon and the precise procedure to glue tubes together will be discussed. The total 
flux $F_{\text {tot }}$ will be the sum of the contributions from each domain wall with the permutation of the symmetries properly considered. Therefore, even when we naively connect a domain wall to a copy of itself, as we are required to permute the symmetries, the flux we shall associate with the resulting domain wall is not twice the flux of the original one. Also when closing a tube on itself, to make a torus compactification, some symmetries may be broken. This then forces the flux to distribute accordingly, eliminating the flux from broken symmetries. As a result the flux associated with the closed surfaces may not be the same as the one associated with the tube when symmetries are broken upon closing the surface.

For combinations of the domain walls which do not satisfy the condition, $\prod_{j=1}^{n} \sigma^{t_{j}}=1$, in particular the basic domain wall, we do not have a suggestion for the Riemann surface it is to be associated with. We merely use the basic walls as building blocks for constructing theories which we can identify with the compactifications. There are several reasons we do not make claims about the basic walls and we will discuss them here. First, we have not found an association of the flux to the basic domain wall such that the anomalies will agree with the six dimensional computation. This can be because either the walls not satisfying the condition do not correspond to compactifications or that there are subtleties with the computation of anomalies we miss. Another issue is that, as we will see soon, there is a natural way to associate flux to the basic blocks such that for surfaces satisfying the conditions given above, the anomalies of the $4 \mathrm{~d}$ theories agree with the computations of anomalies from 6d. This flux however for a single wall is not properly quantized, which again hints that there is an issue with treating basic walls as arising in compactifications. Here we should mention that improperly quantized fluxes for surfaces with punctures have occurred before $[8,10]$. While it is important to resolve the fate of the basic tubes and the way they can be related to compactification, we will leave this for the future. Here we stress again that we only claim the statements appearing in the conjectures. ${ }^{5}$

We also mention that we can construct different looking theories which are associated to the same flux. If our procedure is correct all such theories should be dual to each other. Such examples are easy to construct, for example by gluing tubes with small flux in different order to form a tube with bigger flux in certain cases, and the dualities can be easily checked by computing indices and anomalies. We will refrain from explicitly detailing such computations for the sake of brevity, however we do stress that in a large variety of cases where we made these checks our procedure was found to be consistent.

Below we will provide evidence for these conjectures with examples by comparing anomalies of the $5 \mathrm{~d}$ theory with flux domain walls against the expected anomalies of the $6 \mathrm{~d}$ theory with the corresponding flux.

\footnotetext{
${ }^{5}$ Let us mention in which way fractional fluxes can appear when one considers theories with punctures. In $6 \mathrm{~d}$, we can turn on a flux for the $\mathrm{SU}(k)_{\beta}$ symmetry, like $F=(\overbrace{1 / r, \cdots, 1 / r}^{r},-1 /(k-r), \cdots,-1 /(k-r))$. This flux breaks the $\mathrm{SU}(k)_{\beta}$ symmetry to $\mathrm{U}(1) \times \mathrm{SU}(r) \times \mathrm{SU}(k-r)$. In this case, since the flux is fractional, we also need to turn on center fluxes in the subgroup $\mathrm{SU}(r) \times \mathrm{SU}(k-r)$. These center fluxes lead to a cyclic $\mathbb{Z}_{r} \times \mathbb{Z}_{k-r}$ rotation on the $\mathrm{SU}(r) \times \mathrm{SU}(k-r)$ holonomies. In the 5 d reduction, the flux should be realizable as a domain wall and the corresponding $\mathbb{Z}_{r} \times \mathbb{Z}_{k-r}$ actions become cyclic permutations of $\mathrm{U}(1)^{r} \times \mathrm{U}(1)^{k-r} \subset \mathrm{SU}(k)_{\beta}$ symmetries as we move across the domain wall. The basic domain wall models we constructed behave in many ways like these tubes, for example they give same permutations, yet we do not claim that they are the same models.
} 
Gluing. Let us explain how to connect two flux domain walls with boundary conditions $\mathcal{B}_{1}$ and $\mathcal{B}_{2}$ together. General domain walls can be constructed by repeating this gluing procedure. We consider the first domain wall with boundary condition $\mathcal{B}_{1}$ located at $x^{4}=t_{1}$ and then add the second domain wall with boundary condition $\mathcal{B}_{2}$ at $x^{4}=t_{2}$. First, the vector multiplets in three chambers satisfy Neumann boundary condition, so the theory with the domain walls has $\mathrm{SU}(N)_{1}^{k} \times \mathrm{SU}(N)_{2}^{k} \times \mathrm{SU}(N)_{3}^{k}$ gauge symmetry. The hypermultiplets in the first and the third chambers will couple to the $4 \mathrm{~d}$ chiral fields $q_{i}, \tilde{q}_{i}$ and $q_{i}^{\prime}, \tilde{q}_{i}^{\prime}$ at two interfaces through cubic superpotentials of the form (3.3). Now the $5 \mathrm{~d}$ theory in the second chamber is put on a finite interval between $t_{1}$ and $t_{2}$. So at low energy the theory in the second chamber reduces to a 4 d theory with $\mathrm{SU}(N)_{2}^{k}$ gauge group. The chiral halves of the hypermultiplets satisfying Neumann boundary conditions at both ends reduce to $4 \mathrm{~d}$ chiral multiplets. If a hypermultiplet in the second chamber satisfies opposite boundary conditions at the two ends, this hypermultiplet becomes massive and at low energy they are truncated. After integrating out the massive hypermultiplet, the cubic superpotentials involving this hypermultiplet turn into quartic superpotentials between the 4d chiral fields $q$ and $\tilde{q}$ :

$$
\mathcal{W}^{\prime}=\sum_{i=(+,-)}\left(q_{i+1} \tilde{q}_{i} \tilde{q}_{i}^{\prime} q_{i+1}^{\prime}\right)+\sum_{i=(-,+)}\left(q_{i} \tilde{q}_{i} \tilde{q}_{i}^{\prime} q_{i}^{\prime}\right)
$$

where $i=\left(s_{1}, s_{2}\right)$ runs over the massive hypermultiplets with boundary conditions $s_{a}$ at $t_{a}$.

We shall consider various combinations of basic domain walls aligned along a spatial direction $x^{4}$. The gluing of two basic domain walls can naturally be generalized to the cases with multiple domain walls. In particular, when we identify the first and the last chambers, we will get a $5 \mathrm{~d}$ system compactified on a circle along which a number of basic domain walls are distributed. Note that, when the first and the last chambers are identified, the hypermultiplets in the new chamber reduce to $4 \mathrm{~d}$ chiral fields or are truncated in the same way as those in the second chamber in the two domain wall example above. Thus this system reduces to a $4 \mathrm{~d} \mathcal{N}=1$ quiver gauge theory at low energy. Following the above conjectures, we expect the resulting $4 \mathrm{~d}$ theories implements torus compactifications of the $6 \mathrm{~d}$ theory with fluxes.

Assignment of fluxes. To derive an assignment of flux let us study the structure of the linear anomaly in six dimensions. We here will make the treatment general for $G$ type conformal matter. The fluxes we will discuss are for the Cartan of the $G \times G$ symmetry. For $A$ type we have an additional U(1) symmetry but we do not construct models corresponding to flux for this symmetry. From the anomaly polynomial in six dimensions we obtain that this anomaly in four dimensions is,

$$
\operatorname{Tr} G_{i}=n_{i} N \frac{h^{\vee}}{d_{F}} Q_{i}
$$

Here $Q_{i}$ is the flux for the $\mathrm{U}(1) \operatorname{subgroup} G_{i}$ in $G$ and $n_{i}$ is determined by the embedding of the $\mathrm{U}(1)$ in $G$. Here $N$ is the number of branes probing the singularity. We can absorb $N$ into the definition of $n_{i}$ however, in the way we will normalize the symmetries in all cases, 
$N$ will appear linearly in linear anomaly. On the other hand with a little thought, and we will discuss this in examples below, the only fields contributing to this anomaly in the field theory construction are the flip fields for non-minimal cases. It is thus natural to define the flux in the symmetry $G_{i}$ to be the sum of $G_{i}$ charges of the flip fields. The logic, assuming the theories built from the two punctured spheres and correspond to closed surfaces are the correct ones, and we conjecture they are, is as follows. The gravity anomalies are proportional to the sum of charges of the flip fields for non-minimal cases

$$
\operatorname{Tr} G_{i}=a_{i}^{(G, G)} N \sum_{f} q_{i, f}
$$

Here the sum is over flip fields and $a_{i}^{(G, G)}$ is a constant which depends on the symmetry and the type of conformal matter, we have that

$$
Q_{i}=a_{i}^{(G, G)} \frac{d_{F}}{n_{i} h^{\vee}} \sum_{f} q_{i, f}
$$

That is the flux is the same as sum over charges up to normalization which only depends on the compactification type and the symmetry. Note that the anomaly scales as $N$ in six dimensions and the only fields giving a scaling with $N$ are the flip fields with other behaving quadratically. It is then that in case the models correspond to compactifications the anomalies only come from the flips. For all the cases we studied, we find a rather simple formula for the flux as

$$
Q_{i}=\frac{1}{N h^{\vee}} \sum_{f} q_{i, f}
$$

in the orthogonal basis of the flavor symmetry $G \times G^{\prime}$ which is the basis we will use in this section for the flavor symmetries of ADE conformal matters.

The fluxes for minimal cases, on the other hand, are not solely determined by the charges of flip fields. Here we shall instead use the full linear anomalies, where the flux is chosen such that the linear anomalies of the $4 \mathrm{~d}$ theories match those expected from $6 \mathrm{~d}$. For example, the flux of a basic domain wall can be determined by using the $4 \mathrm{~d}$ tube theory with this domain wall. We compare the linear anomalies of this tube theory with those of the $6 \mathrm{~d}$ theory on a tube involving both the geometric contributions and the puncture contributions which we will discuss in detail soon. Although we do not expect this tube theory matches the compactification of the $6 \mathrm{~d}$ theory since $\sigma \neq 1$ for this case, we use this comparison to fix the flux of the basic domain wall.

We claim that with this identification of flux the anomalies for tori match between all the different computations both for minimal and non-minimal cases. This will be true for any closed Riemann surface if the total flux is integer in proper sense. If it is not then the anomaly only agrees for components of symmetry which have integer flux.

Our basic domain walls carry fluxes only on either $G_{\beta}$ or on $G_{\gamma}$ of the $G_{\beta} \times G_{\gamma}$ symmetry depending on the representations of the $4 \mathrm{~d}$ chiral fields $q_{i}$ denoted by $\mathcal{T}=\beta, \gamma$, and the explicit form of $Q_{i}$ is fixed by the boundary condition $\mathcal{B}=\{ \pm, \pm, \cdots, \pm\}$. So we 


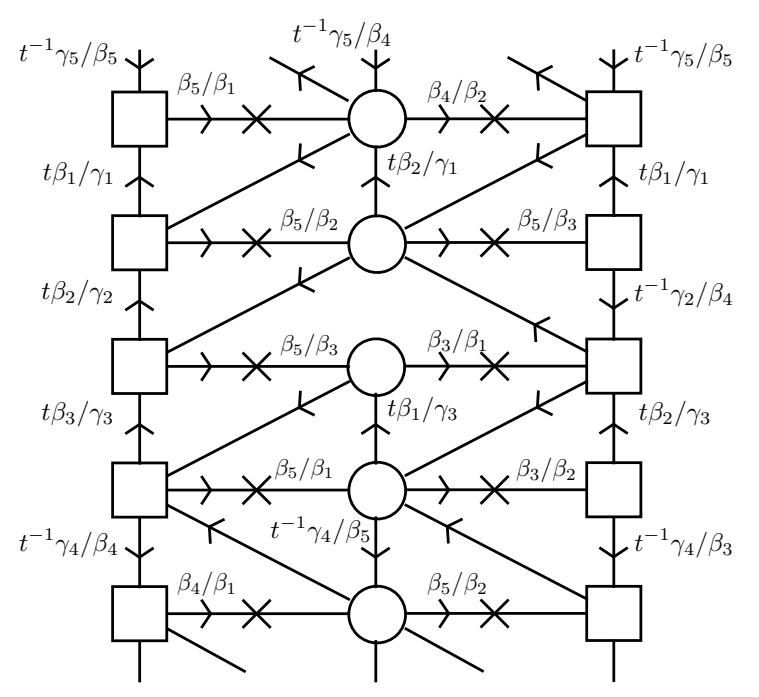

Figure 4. Gluing two domain walls with $\mathcal{D}_{1}=\{+,+,+,-,-\}_{\beta}$ and $\mathcal{D}_{2}=\{+,-,+,-,-\}_{\beta}$. The total flux of the final domain wall is $F_{\text {tot }}=(3 / 5,1 / 5,1 / 5,-1 / 5,-4 / 5)_{\beta}+(2 / 5,3 / 5,-2 / 5$, $-2 / 5,-1 / 5)_{\beta}=(1,4 / 5,-1 / 5,-3 / 5,-1)_{\beta}$.

will label the basic domain walls by $\mathcal{D}=\mathcal{B}_{\mathcal{T}}$. General flux domain walls carrying both $G_{\beta}$ and $G_{\gamma}$ fluxes can be built by joining flux domain walls of two types $\mathcal{T}=\beta$ and $\mathcal{T}=\gamma$.

We will now discuss examples of compactifications of different types of conformal matter. We will discuss the prescription to associate theories to surfaces in more detail and give examples of various checks one can perform.

More general models and useful examples. For example, when we connect two domain walls in figure 3 , we get a bigger domain wall with flux $F=(1,4 / 5,-1 / 5,-3 / 5,-1)_{\beta}$ for $\mathrm{SU}(5)_{\beta}$ drawn in figure 4 . Note that the flip fields of the left diagram in figure 3 have charges $\left(q_{\beta_{1}}, q_{\beta_{2}}, q_{\beta_{3}}, q_{\beta_{4}}, q_{\beta_{5}}\right)$ as

$$
(N, 0,0,0,-N),(0, N, 0,0,-N),(0,0, N, 0,-N),(N, 0,0,0,-N),(N, 0,0,-N, 0),
$$

from the top to the bottom, respectively, and those of the right diagram have charges

$$
(N, 0,0,0,-N),(0,0, N, 0,-N),(0,-N, N, 0,0),(N,-N, 0,0,0),(N, 0,0,-N, 0)
$$

respectively. When we combine these two domain walls as in figure 4 and compute the total flux for it, we should take into account the permutation of the $\mathrm{U}(1)_{\beta_{i}}$ symmetry. So, regarding the permutations $\sigma_{\beta}^{1}=\left(\begin{array}{lll}1 & 2 & 3\end{array}\right)(45), \sigma_{\beta}^{2}=\left(\begin{array}{ll}1 & 3\end{array}\right)(254)$ and using the flux relation given in (3.14) with $h^{\vee}=5$, we obtained the total flux $F=(1,4 / 5,-1 / 5,-3 / 5,-1)_{\beta}$ for the domain wall in figure 4 . Three or more domain walls can also be connected together by using the above gluing rules for each pair of adjacent domain walls. Also, by identifying two $5 \mathrm{~d}$ theories in the first and the last chambers, we can construct the $5 \mathrm{~d} A_{k}$ quiver gauge theory on a circle with flux domain walls that corresponds to the $6 \mathrm{~d}$ theory compactified on a torus with flux. 

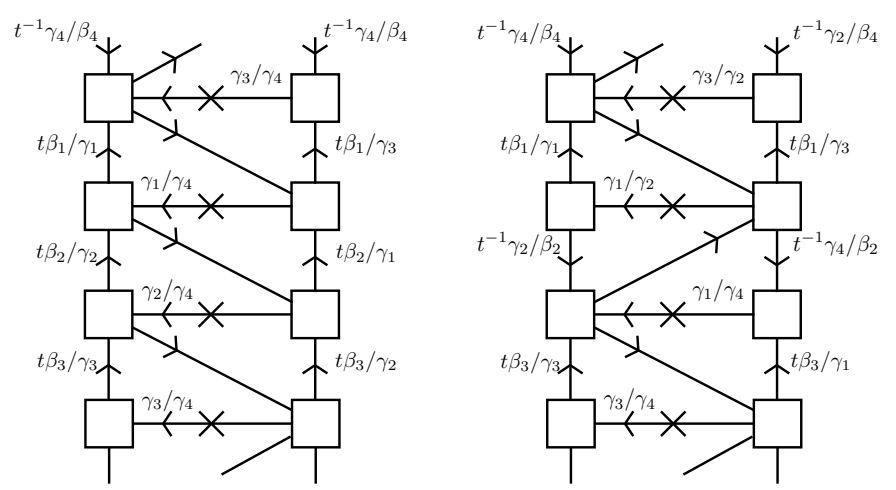

Figure 5. Domain walls of type $\mathcal{T}=\gamma$ related to $\mathrm{SU}(k)_{\gamma}$ fluxes. The left one is related to the flux $F=(-1 / 4,-1 / 4,-1 / 2,1)_{\gamma}$ and the right one is related to the flux $F=(-1 / 2,1 / 2,-1 / 2,1 / 2)_{\gamma}$ in the $6 \mathrm{~d} \mathrm{SU}(k)_{\gamma}$ symmetry.

So far we discussed the domain walls of type $\mathcal{T}=\beta$ with fluxes only on the $\mathrm{SU}(k)_{\beta}$ symmetry. We can construct the domain walls of type $\mathcal{T}=\gamma$ for $\mathrm{SU}(k)_{\gamma}$ flux in a similar way. As discussed above, the main difference for a given boundary condition $\mathcal{B}$ is the representation of the $4 \mathrm{~d}$ chiral fields $q_{i}$. We flip the representation of $q_{i}$ from $(\overline{\mathbf{N}}, \mathbf{N})$ to $(\mathbf{N}, \overline{\mathbf{N}})$ of the $\mathrm{SU}(N)_{i} \times \mathrm{SU}(N)_{i}^{\prime}$ gauge symmetry. It then follows that the interface hosts the following superpotentials:

$$
\mathcal{W}_{x^{4}=0}=\sum_{i=+}\left(q_{i+1} \tilde{q}_{i} X_{i}+\tilde{q}_{i} q_{i} X_{i}^{\prime}\right)+\sum_{i=-}\left(Y_{i} q_{i} \tilde{q}_{i}+Y_{i}^{\prime} \tilde{q}_{i} q_{i+1}\right)
$$

The representations of the other chiral fields $\tilde{q}_{i}$ need to be chosen accordingly. We also add flip chiral fields coupled to the baryonic operators of $q_{i}$ 's. One can easily check that this domain wall configuration has no cubic gauge anomalies and also that all $\mathrm{U}(1)$ charges for the $4 \mathrm{~d}$ fields are uniquely fixed with no additional abelian symmetry other than $\mathrm{U}(1)_{\beta_{i}} \times \mathrm{U}(1)_{\gamma_{i}} \times \mathrm{U}(1)_{t}$ symmetries. Two examples in the $A_{3}$ quiver gauge theory are depicted in figure 5 .

We define this type of domain walls with $\mathcal{B}_{\gamma}$ as the basic flux domain walls with flux $F=\left(n_{1}, n_{2}, \cdots, n_{k}\right)$ for the $\mathrm{SU}(k)_{\gamma}$ symmetry where $n_{i}=-1 / r$ for $s_{i}=+$ or $n_{i}=1 /(k-r)$ for $s_{i}=-$, and $r$ is the number of $+\operatorname{signs}$ in $\mathcal{B}$. Note that this domain wall permutes cyclically $\mathrm{U}(1)_{\gamma}^{r}$ and $\mathrm{U}(1)_{\gamma}^{k-r}$ symmetries respectively. More precisely, the $\sigma_{\gamma}(\mathcal{B})$ is the counterclockwise permutation of $\mathrm{U}(1)_{\gamma_{i}}$ symmetries with $s_{i}=+$ and the clockwise permutation of $\mathrm{U}(1)_{\gamma_{i}}$ symmetries with $s_{i}=-$, and $\sigma_{\beta}(\mathcal{B})=1$. Following the conjectures above, we propose that a domain wall configuration constructed by these domain walls realize the flux compactification of the $6 \mathrm{~d}$ theory when $\prod_{i} \sigma^{t_{i}}=1$ or when the system is compactified on a circle (so when the $6 \mathrm{~d}$ theory is put on a torus).

For more general fluxes in both $\mathrm{SU}(k)_{\beta}$ and $\mathrm{SU}(k)_{\gamma}$ symmetries, we can simply combine the domain walls for $\mathrm{SU}(k)_{\beta}$ flux with the domain walls of the second type for $\mathrm{SU}(k)_{\gamma}$ flux. Gluing these two different types of domain walls is straightforward. As the cases above, we will have a new chamber between two domain walls and at low energy the $5 \mathrm{~d}$ theory in this chamber reduces to a $4 \mathrm{~d}$ theory. The hypermultiplets with the same boundary conditions 


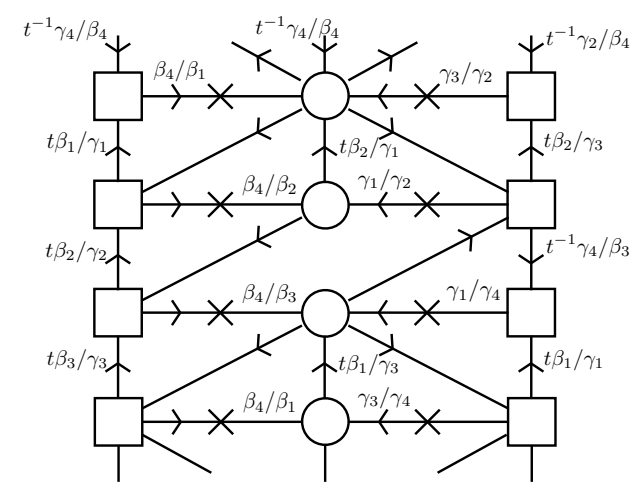

Figure 6. A combination of a domain wall with $\mathrm{SU}(k)_{\beta}$ flux and a domain wall with $\mathrm{SU}(k)_{\gamma}$ flux in the affine $A_{3}$ quiver gauge theory. The total flux becomes $F_{\text {tot }}=(1 / 2,1 / 4,1 / 4,-1)_{\beta}+$ $(-1 / 2,1 / 2,-1 / 2,1 / 2)_{\gamma}$. The top gauge group has four $N$ flavors, which means it is free in the IR. The way to think about this theory is to perform a Seiberg duality on the second node which will remove $N$ flavors from the first gauge groups. The resulting theory is free in the ultra violet. The theories we consider might have complicated dynamics when we flow to the IR. In all examples we consider there is a way to make sense of the models as complete in the ultra-violet.

at the two ends leave $4 \mathrm{~d}$ chiral fields coupled to the degrees of freedom at the interfaces and integrating out massive hypers with opposite boundary conditions at the two ends induces quartic superpotential couplings as discussed above. An example of gluing a flux domain wall of type $\mathcal{T}=\beta$ and another flux domain wall with $\mathcal{T}=\gamma$ in the $A_{3}$ quiver theory is given in figure 6 . Here, there is a quartic superpotential of the form $q_{3} \tilde{q}_{2} q_{2}^{\prime} \tilde{q}_{2}^{\prime}$ where $q, \tilde{q}$ are the $4 \mathrm{~d}$ chiral fields in the first domain wall and $q^{\prime}, \tilde{q}^{\prime}$ are the $4 \mathrm{~d}$ fields in the second domain wall.

$4 \mathrm{~d}$ reduction and punctures. Let us now compare our $5 \mathrm{~d}$ domain wall configurations and the $6 \mathrm{~d}$ theory with fluxes on Riemann surfaces. We first consider the $4 \mathrm{~d}$ reduction of the $6 \mathrm{~d}$ theory compactified on a tube (or a two punctured sphere). We can compute 't Hooft anomalies of the resulting $4 \mathrm{~d}$ theories from the $6 \mathrm{~d}$ anomaly polynomial by integrating it on a tube. In addition, there are anomaly inflow contributions from the $6 \mathrm{~d}$ bulk theory toward two punctures. We will call the former as the geometric contribution and the latter as the inflow contribution $[10,11]$. By adding these two contributions, we can compute the total 't Hooft anomalies of the $4 \mathrm{~d}$ compactification. The geometric contribution can be computed using the method studied in section 2. The inflow contribution can be obtained from the 5d quiver gauge theories ending on a boundary. We will now explain how to compute this inflow contribution. See $[10,11]$ for more discussions.

We can deform the $6 \mathrm{~d}$ theory near a puncture as a long and thin tube ending on a boundary. Since we topologically twist the $6 \mathrm{~d}$ theory on the $2 \mathrm{~d}$ surface, this deformation has no effect in the $4 \mathrm{~d}$ reduction. The $6 \mathrm{~d}$ theory around the puncture at low energy reduces to the $5 \mathrm{~d}$ affine quiver gauge theory ending on the boundary. This picture suggests a one-to-one correspondence between the type of punctures and the choice of boundary conditions in the $5 \mathrm{~d}$ theory. Thus, for a given puncture on a Riemann surface, we can find the corresponding boundary condition. This boundary condition leads to additional 't Hooft anomalies in the $4 \mathrm{~d}$ theory through the inflow mechanism. 
So punctures on a Riemann surface are associated to boundary conditions in the $5 \mathrm{~d}$ theory. In this work, we will focus only on maximal punctures, which are defined by boundary conditions similar to those appearing in the domain walls, and without additional $4 \mathrm{~d}$ degrees of freedom at the boundary. These are so named as they generalize the maximal punctures appearing in class $\mathcal{S}$ theories to the case of generic $A D E$ group. These types of punctures depend on a discrete parameter, called color, denoted by the permutations among the Cartans of $G \times G^{\prime}$ symmetry. This additional degree of freedom comes from the option of performing $G \times G^{\prime}$ Weyl transformations. The puncture with this boundary condition is defined as follows.

We first give Dirichlet boundary condition to the vector multiplets, so the gauge symmetries in the bulk $5 \mathrm{~d}$ theory become $4 \mathrm{~d}$ global symmetries at the boundary. This endows the puncture with an affine quiver type global symmetry in addition to the $G \times G^{\prime}$ symmetry. The hypermultiplets satisfy the standard $1 / 2$ BPS boundary condition $\mathcal{B}$ defined in (3.2), and thus they give rise to $4 \mathrm{~d}$ chiral multiplets charged under the affine quiver global symmetry of the puncture. When each hypermultiplet leaves a $4 \mathrm{~d}$ chiral multiplet at the $4 \mathrm{~d}$ boundary, we will call this type of punctures as maximal punctures for any $5 \mathrm{~d}$ affine quiver gauge theory.

The boundary conditions for the $5 \mathrm{~d}$ theory induce anomaly inflows toward the maximal puncture and thus the punctures in general carry non-trivial anomalies. These anomalies depend on the boundary condition $\mathcal{B}$ and can be considered as a defining property of the punctures. In particular, two or more maximal punctures for a $6 \mathrm{~d}$ theory have the same type of affine quiver global symmetries, but, due to the permutations by flux, they can have different colors with respect to the Cartan of $G \times G^{\prime}$ symmetry. This results in different 't Hooft anomalies of the punctures.

As we studied above, the anomaly inflows consist of matter contributions and the gauge kinetic term (or gauge-global mixed Chern-Simons term) contributions. The hypermultiplet contributions and the kinetic contributions are the same as before. As explained above, a chiral fermion from a $5 \mathrm{~d}$ hypermultiplet with Neumann boundary condition induces half of the anomalies from a $4 \mathrm{~d}$ chiral fermion with the same charges. Also, the gauge kinetic terms provide inflow contributions for mixed anomalies between the affine quiver global symmetry and subsets of $G \times G^{\prime}$ associated to the instanton symmetry as (3.7). That is for the gauge group $G_{i}$

$$
\operatorname{Tr}\left(\mathrm{U}(1) G_{i}^{2}\right)=\frac{1}{2} Q_{i}
$$

where $Q_{i}$ is the $\mathrm{U}(1)$ global charge of a unit instanton state. On the other hand, the vector multiplets now satisfy Dirichlet boundary condition. So their inflow contributions are minus of those for the Neumann boundary condition, which we compute

$$
\operatorname{Tr}\left(\mathrm{U}(1)_{R}\right)=\operatorname{Tr}\left(\mathrm{U}(1)_{R}^{3}\right)=-\frac{d_{G_{i}}}{2}, \quad \operatorname{Tr}\left(\mathrm{U}(1)_{R} G_{i}^{2}\right)=-\frac{h^{\vee}}{2},
$$

from the vector multiplet of the gauge group $G_{i}$. Collecting all these contributions, we can compute the anomalies assigned for a maximal puncture.

Let us discuss some more details of the punctures in the $\left(A_{k-1}, A_{k-1}\right)$ conformal matter theory. A maximal puncture in this theory supports $\mathrm{SU}(N)^{k}$ global symmetry. The anoma- 
lies of this puncture can be computed as follows. The vector multiplets induce anomaly inflows given by

$$
\operatorname{Tr}\left(\mathrm{U}(1)_{R}\right)=\operatorname{Tr}\left(\mathrm{U}(1)_{R}^{3}\right)=-\frac{k\left(N^{2}-1\right)}{2}, \quad \operatorname{Tr}\left(\mathrm{U}(1)_{R} \mathrm{SU}(N)_{i}^{2}\right)=-\frac{N}{2} \text { for all } i
$$

The $i$-th hypermultiplet provides the inflow contributions as

$$
\begin{aligned}
\operatorname{Tr}\left(\mathrm{U}(1)_{t}\right) & =\sum_{i} \frac{s_{i} N^{2}}{2}, \quad \operatorname{Tr}\left(\mathrm{U}(1)_{\beta_{i}}\right)=\frac{s_{i} N^{2}}{2}, \quad \operatorname{Tr}\left(\mathrm{U}(1)_{\gamma_{i}}\right)=-\frac{s_{i} N^{2}}{2}, \\
\operatorname{Tr}\left(\mathrm{U}(1)_{t} \mathrm{SU}(N)_{i}^{2}\right) & =\frac{\left(s_{i}+s_{i-1}\right) N}{4}, \quad \operatorname{Tr}\left(\mathrm{U}(1)_{\beta_{i}} \mathrm{SU}(N)_{i}^{2}\right)=\operatorname{Tr}\left(\mathrm{U}(1)_{\beta_{i}} \mathrm{SU}(N)_{i+1}^{2}\right)=\frac{s_{i} N}{4}, \\
\operatorname{Tr}\left(\mathrm{U}(1)_{\gamma_{i}} \mathrm{SU}(N)_{i}^{2}\right) & =\operatorname{Tr}\left(\mathrm{U}(1)_{\gamma_{i}} \mathrm{SU}(N)_{i+1}^{2}\right)=-\frac{s_{i} N}{4}, \\
\operatorname{Tr}\left(\mathrm{U}(1)_{a} \mathrm{U}(1)_{b} \mathrm{U}(1)_{c}\right) & =\sum_{i} \frac{s_{i} Q_{a} Q_{b} Q_{c} N^{2}}{2} \quad\left(\text { with } a, b, c \in\left\{t, \beta_{i}, \gamma_{i}\right\}\right), \\
\operatorname{Tr}\left(\mathrm{SU}(N)_{i}^{3}\right) & =\left(s_{i}-s_{i-1}\right) \frac{N}{2},
\end{aligned}
$$

where $s_{i}$ is the boundary condition and $Q_{a}$ denotes the $\mathrm{U}(1)_{a}$ global charge of the $i$-th hypermultiplet. Also the $\mathrm{SU}(N)^{k}$ Yang-Mills terms provide additional contributions as

$$
\begin{gathered}
\operatorname{Tr}\left(\mathrm{U}(1)_{\beta_{i}} \mathrm{SU}(N)_{i}^{2}\right)=\operatorname{Tr}\left(\mathrm{U}(1)_{\gamma_{i}} \mathrm{SU}(N)_{i}^{2}\right)=\frac{N}{4}, \\
\operatorname{Tr}\left(\mathrm{U}(1)_{\beta_{i}} \mathrm{SU}(N)_{i+1}^{2}\right)=\operatorname{Tr}\left(\mathrm{U}(1)_{\gamma_{i}} \mathrm{SU}(N)_{i+1}^{2}\right)=-\frac{N}{4} .
\end{gathered}
$$

Then the full anomaly inflow for a maximal puncture with $\mathcal{B}$ on a Riemann surface is given by a sum over these inflow contributions. We note that proper permutations $\left(\sigma_{\beta}, \sigma_{\gamma}\right)$ should be taken into account when there are two or more punctures.

Consider now the $5 \mathrm{~d}$ affine $A_{k-1}$ quiver gauge theory with domain walls on an interval $0<I<L$. We impose maximal boundary conditions giving maximal punctures at $x^{4}=$ $0, L$. At low energy $E \ll \frac{1}{L}$, this theory reduces to a $4 \mathrm{~d} \mathcal{N}=1$ quiver gauge theory. The resulting $4 \mathrm{~d}$ theory will have $\mathrm{SU}(N)^{k} \times \mathrm{SU}(N)^{\prime k}$ global symmetries arising from the Dirichlet boundary conditions at $x^{4}=0, L$ and $\mathrm{U}(1)_{\beta}^{k-1} \times \mathrm{U}(1)_{\gamma}^{k-1} \times \mathrm{U}(1)_{t}$ flavor symmetries from the hypermultiplets. We propose that this $4 \mathrm{~d}$ theory, when $\prod_{i} \sigma^{t_{i}}=1$, corresponds to the $6 \mathrm{~d}\left(A_{k-1}, A_{k-1}\right)$ theory with fluxes on a tube with maximal punctures at both ends.

For example, we can engineer a $4 \mathrm{~d}$ quiver gauge theory by connecting two basic domain walls of $\mathcal{D}=\{+,+,-\}_{\beta}$ in the $5 \mathrm{~d}$ affine $A_{2}$ theory as drawn in figure 7 . The fluxes associated with these domain walls are $F_{1}=(2 / 3,1 / 3,-1)_{\beta}, F_{2}=(1 / 3,2 / 3,-1)_{\beta}$, respectively, and their combination gives a flux of $F=(1,1,-2)_{\beta}$. Note that the combination of the two permutations becomes trivial, i.e. $\sigma^{t_{1}} \sigma^{t_{2}}=1$ where $\sigma^{t_{1}}=\sigma^{t_{2}}=\left(\begin{array}{ll}1 & 2\end{array}\right)_{\beta}$. We thus propose that this $4 \mathrm{~d}$ theory is the $6 \mathrm{~d}\left(A_{2}, A_{2}\right)$ conformal matter theory on a tube with two maximal punctures and flux $F=(1,1,-2)_{\beta}$. 


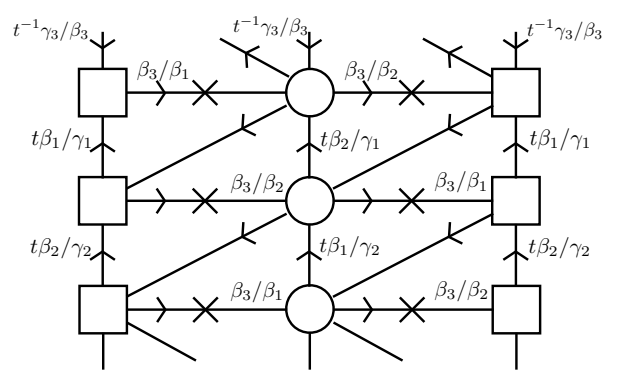

Figure 7. Flux domain wall for $F_{\text {tot }}=(1,1,-2)_{\beta}$ in the affine $A_{2}$ quiver theory. The permutations in the first and second domain walls are $\sigma^{t_{1}}=\sigma^{t_{2}}=\left(\begin{array}{ll}1 & 2\end{array}\right)$ and thus $\sigma^{t_{1}} \sigma^{t_{2}}=1$.

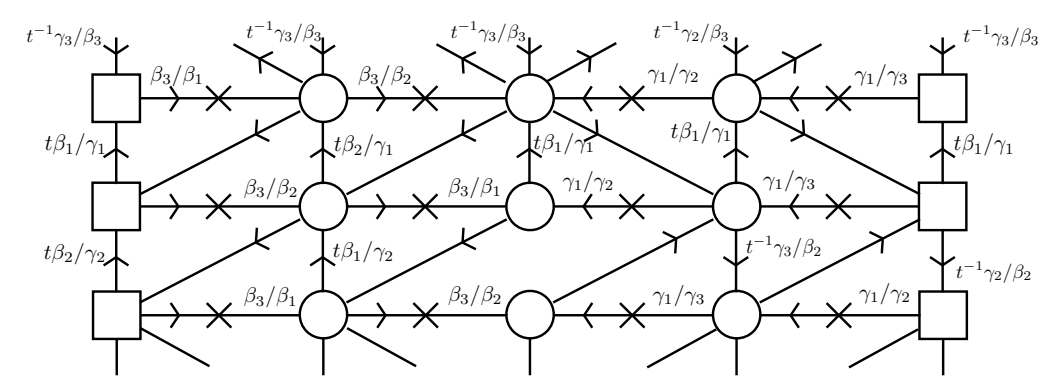

Figure 8. A combination of four flux domain wall with $F_{\text {tot }}=(1,1,-2)_{\beta}+(-2,1,1)_{\gamma}$ in the affine $A_{2}$ quiver theory. The permutations in four domain walls are $\sigma^{t_{1}}=\sigma^{t_{2}}=\left(\begin{array}{ll}1 & 2\end{array}\right)_{\beta}$ and

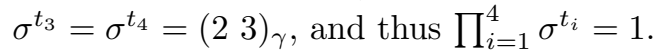

Combining the geometric contribution, which is given by

$$
\begin{aligned}
& \operatorname{Tr}\left(\mathrm{U}(1)_{\beta_{1,2}}\right)=9 N, \quad \operatorname{Tr}\left(\mathrm{U}(1)_{R}^{2} \mathrm{U}(1)_{\beta_{1,2}}\right)=-27\left(N^{2}-1\right), \quad \operatorname{Tr}\left(\mathrm{U}(1)_{t}^{2} \mathrm{U}(1)_{\beta_{1,2}}\right)=27 N^{2} \\
& \operatorname{Tr}\left(\mathrm{U}(1)_{t} \mathrm{U}(1)_{\beta_{1,2}}^{2}\right)=-9 N^{2}, \quad \operatorname{Tr}\left(\mathrm{U}(1)_{t} \mathrm{U}(1)_{\beta_{1}} \mathrm{U}(1)_{\beta_{2}}\right)=-36 N^{2}, \\
& \operatorname{Tr}\left(\mathrm{U}(1)_{\beta_{1,2}}^{3}\right)=9\left(3 N^{3}-2 N^{2}\right), \quad \operatorname{Tr}\left(\mathrm{U}(1)_{\beta_{1}}^{2} \mathrm{U}(1)_{\beta_{2}}\right)=\operatorname{Tr}\left(\mathrm{U}(1)_{\beta_{2}}^{2} \mathrm{U}(1)_{\beta_{1}}\right)=18\left(3 N^{3}-2 N^{2}\right) \\
& \operatorname{Tr}\left(\mathrm{U}(1)_{\beta_{1,2}} \mathrm{U}(1)_{\gamma_{a}} \mathrm{U}(1)_{\gamma_{b}}\right)=18 N^{2} \quad \text { for } a=1,2,
\end{aligned}
$$

and the inflow contributions written in $(3.20),(3.21),(3.22)$ with $\left(s_{1}, s_{2}, s_{3}\right)=(+,+,-)$ for the two punctures, we find the anomalies of the $6 \mathrm{~d}$ theory on a tube perfectly agree with the 't Hooft anomalies of the $4 \mathrm{~d}$ quiver gauge theory in figure 7 . Also, one can easily show that the $4 \mathrm{~d}$ quiver gauge theory obtained by combining any number $n \in 2 \mathbb{Z}$ of the basic domain walls for $\mathcal{D}=\{+,+,-\}_{\beta}$ has the same 't Hooft anomalies as those from the $6 d$ theory with flux $F=n(1 / 2,1 / 2,-1)_{\beta}$ and two maximal punctures.

A more complicated example with $\mathcal{D}_{1}=\mathcal{D}_{2}=\{+,+,-\}_{\beta}$ and $\mathcal{D}_{3}=\mathcal{D}_{4}=\{+,-,-\}_{\gamma}$ is given in figure 8 . We expect that this $4 \mathrm{~d}$ quiver gauge theory corresponds to the $6 \mathrm{~d}$ $\left(A_{2}, A_{2}\right)$ theory on a tube with flux $F_{\text {tot }}=(1,1,-2)_{\beta}+(-2,1,1)_{\gamma}$. In this case, two punctures amount to two different boundary conditions, $\mathcal{B}_{1}=\{+,+,-\}$ and $\mathcal{B}_{2}=\{+,-,-\}$ respectively. We checked that the 't Hooft anomalies of this $4 \mathrm{~d}$ theory agree with the geometric and inflow results of the $6 \mathrm{~d}$ theory with the $F_{\text {tot }}$ and these two punctures.

We now consider gluing two boundaries of the $5 \mathrm{~d}$ quiver theory on an interval in the presence of domain walls. From $5 \mathrm{~d}$ perspective, this gluing can be simply considered as 
identifying the first and the last chambers without boundaries. Or we can also consider this as connecting two $5 \mathrm{~d}$ theories in the first and the last chamber by a trivial interface between them. The $4 \mathrm{~d}$ viewpoint of gluing two punctures will be presented in the next section. At low energy after gluing two ends of the $5 \mathrm{~d}$ theory, we will have a $4 \mathrm{~d}$ quiver gauge theory. We conjecture that this $4 \mathrm{~d}$ theory realizes a torus compactification the $6 \mathrm{~d}\left(A_{k-1}, A_{k-1}\right)$ conformal matter theory with flux. We expect that this conjecture, for the $6 \mathrm{~d}$ theory on a torus, holds also for more general domain wall configurations with $\prod_{i} \sigma^{t_{i}} \neq 1$. When $\prod_{i} \sigma^{t_{i}} \neq 1$, the corresponding $6 \mathrm{~d}$ theory has fractional flux with non-trivial center flux. This fractional flux breaks some subsets of $\mathrm{SU}(k)_{\beta} \times \mathrm{SU}(k)_{\gamma}$ symmetries. The same symmetry breaking occurs in the 5 d quiver theory on a circle when the global symmetries in the first chamber and those in the last chamber are identified due to the non-trivial permutation. In the next section, we will see a number of examples of $4 \mathrm{~d}$ quiver theories corresponding to the $6 \mathrm{~d}$ theory on a torus with various fluxes and test them using superconformal indices and anomaly matchings.

We remark here that our domain wall construction fails to realize the compactifications of $6 \mathrm{~d}$ conformal matters on a tube with fractional fluxes. The $4 \mathrm{~d}$ theories we obtain using our domain walls on an interval with fractional fluxes have wrong 't Hooft anomalies against the expected anomalies of the compactification of the $6 \mathrm{~d}$ theories. This may imply that our flux domain wall is not the correct domain wall for $6 \mathrm{~d}$ fractional fluxes. We may have missed some $4 \mathrm{~d}$ degrees of freedom and associated superpotentials at the interfaces, but they disappear or decouple when we combine domain walls so that $\prod_{i} \sigma^{t_{i}}$ becomes trivial or when we locate the domain walls on a circle. Another possibility is that the $6 \mathrm{~d}$ flux leaves non-trivial Chern-Simons terms for the global symmetries in the $5 \mathrm{~d}$ reduction in the presence of flavor holonomies. These $5 \mathrm{~d}$ Chern-Simons terms do not affect the dynamics of the $5 \mathrm{~d}$ gauge theory, but they may induce additional inflow contributions toward the $4 \mathrm{~d}$ boundaries. We leave further investigations on this mismatch to future research.

Generalization to $\mathbf{D}$ and E. The same idea in this subsection will be used to build the D-type and E-type domain walls below. For these cases, the symmetry $G \times G^{\prime}$ at the interface will be different, but, apart from this, all other ingredients will be essentially identical. All domain walls will be constructed by first specifying boundary conditions $\mathcal{B}=\{ \pm, \pm, \cdots, \pm\}$ for the $5 \mathrm{~d}$ hypermultiplets and then coupling them to the $4 \mathrm{~d}$ chiral multiplets $q_{i}$ and $\tilde{q}_{i}$ and flip fields. The $4 \mathrm{~d}$ bifundamental field $q_{i}$ is in either a $G_{i} \rightarrow G_{i}^{\prime}$ representation or a $G_{i} \leftarrow G_{i}^{\prime}$ representation and these two choices will be denoted by $\mathcal{T}=\beta$ or $\gamma$, respectively. All quiver nodes are connected to each other through the cubic superpotentials of the form in (3.3). The representations of the other $4 \mathrm{~d}$ fields $\tilde{q}_{i}$ are fixed by the boundary condition $\mathcal{B}$ and the superpotential terms accordingly. When the quiver node involves $\mathrm{U}(1)$ gauge symmetries, we replace them by two fundamental hypermultiplets of the adjacent $\mathrm{SU}(2)$ gauge nodes. In this case, we will add another cubic term like $\tilde{W}=X q X^{\prime}$ between the chiral fields $X$ and $X^{\prime}$ coming from the $\mathrm{SU}(2)$ fundamentals and $\mathrm{SU}(2)^{\prime}$ fundamentals in the two chambers. Abelian charges of the $4 \mathrm{~d}$ chiral multiplets are fixed by the gauge-global mixed anomaly cancellation and the superpotentials. In particular the $6 \mathrm{~d} \mathrm{U}(1)_{R}$ charges of the $4 \mathrm{~d}$ chiral multiplets $q_{i}$ and $\tilde{q}_{i}$ are always fixed to be 0 and +1 
respectively. The resulting domain walls labelled by $\mathcal{D}=\mathcal{B}_{\mathcal{T}}$ turn out to have no cubic gauge anomalies, therefore they can consistently couple to the $5 \mathrm{~d}$ boundary conditions without introducing additional flavor symmetries. We expect the same conjectures hold for D- and E-type domain walls which we will discuss now.

\section{$3.2 \quad$ D-type domain walls}

Let us now turn to the construction of flux domain walls in the $5 \mathrm{~d}$ reductions of $6 \mathrm{~d}$ D-type conformal matter theories. The $5 \mathrm{~d}$ theory without the domain walls is an affine $D_{k+3}$ quiver gauge theory with $\mathrm{SU}(N)^{2} \times \mathrm{SU}(2 N)^{k} \times \mathrm{SU}(N)^{2}$ gauge group. When $N=1$ the vertical lines at the edge of the quiver become free fields which form a mass term with the flip fields, as the $\mathrm{SU}(1)$ gauge groups are empty. In these cases the $\mathrm{SU}(1)^{2} \times \mathrm{SU}(1)^{2}$ gauge nodes at the two ends of the quiver can be replaced by four fundamental hypermultiplets for the first $\mathrm{SU}(2)$ gauge node and another four fundamentals for the last $\mathrm{SU}(2)$ gauge node. We will discuss the cases for $N=1$ separately at the end of this section.

The $6 \mathrm{~d}$ global symmetry $\mathrm{SO}(2 k+6)_{\beta} \times \mathrm{SO}(2 k+6)_{\gamma}$ is broken by non-zero holonomies to $\mathrm{U}(1)^{2 k+6}=\prod_{i=1}^{k+3} \mathrm{U}(1)_{\beta_{i}} \times \mathrm{U}(1)_{\gamma_{i}}$ Cartans and the remaining abelian symmetries are mapped to certain combinations of the flavor symmetries acting on the bifundamental hypers and the topological instanton symmetries in the 5 d gauge theory. In our notation, the bifundamental hypermultiplets $\Phi_{i}=\left(X_{i}, Y_{i}\right)$ carry the $\mathrm{U}(1)^{2 k+6}$ charges $Q_{i}$ as follows:

$$
\begin{aligned}
& \Phi_{1}: \quad\left(Q_{\beta_{1}}, Q_{\beta_{k+2}}, Q_{\gamma_{1}}, Q_{\gamma_{k+2}}\right)=\frac{1}{2}(1,1,-1,-1), \\
& \Phi_{2}: \quad\left(Q_{\beta_{1}}, Q_{\beta_{k+2}}, Q_{\gamma_{1}}, Q_{\gamma_{k+2}}\right)=\frac{1}{2}(1,-1,-1,1), \\
& \Phi_{i}: \quad\left(Q_{\beta_{i-1}}, Q_{\gamma_{i-1}}\right)=\frac{1}{2}(1,-1) \quad \text { for } \quad 3 \leq i \leq k+1, \\
& \Phi_{k+2}: \quad\left(Q_{\beta_{k+1}}, Q_{\beta_{k+3}}, Q_{\gamma_{k+1}}, Q_{\gamma_{k+3}}\right)=\frac{1}{2}(1,1,-1,-1), \\
& \Phi_{k+3}: \quad\left(Q_{\beta_{k+1}}, Q_{\beta_{k+3}}, Q_{\gamma_{k+1}}, Q_{\gamma_{k+3}}\right)=\frac{1}{2}(1,-1,-1,1) .
\end{aligned}
$$

Here, the fields $\Phi_{1}, \Phi_{2}, \Phi_{k+2}, \Phi_{k+3}$ are in the fundamental represntations of four $\operatorname{SU}(N)$ gauge groups, which we will denote by $\mathrm{SU}(N)_{1,2,3,4}$, respectively.

The flux in the $6 \mathrm{~d}$ theory is expected to be realized as a certain domain wall configuration in this $5 \mathrm{~d}$ theory. We will first propose basic domain walls and then construct general flux domain walls by gluing a series of basic domain walls in the appropriate manner. As discussed the domain wall construction in the D-type quiver theory is similar to that of the A-type theory. The domain wall comes with a $4 \mathrm{~d}$ interface between two $5 \mathrm{~d}$ affine $D_{k+3}$ quiver gauge theories, and $4 \mathrm{~d}$ degrees of freedom and superpotentials at the interface linking boundary conditions of two $5 \mathrm{~d}$ theories on both sides of the wall.

The $1 / 2$ BPS boundary condition at the interface $\left(x^{4}=0\right)$ is the same as that in the Atype domain wall dicussed before. The vector multiplets of the $\mathrm{SU}(N)^{2} \times \mathrm{SU}(2 N)^{k} \times \mathrm{SU}(N)^{2}$ gauge group satisfy the Neumann boundary condition defined in equation (3.1). Thus we will have $\left(\mathrm{SU}(N)^{2} \times \mathrm{SU}(2 N)^{k} \times \mathrm{SU}(N)^{2}\right)^{2}$ gauge symmetries at the interface. The $i$-th bifundamental hypermultiplet satisfies the boundary condition in equation (3.2) labelled by 

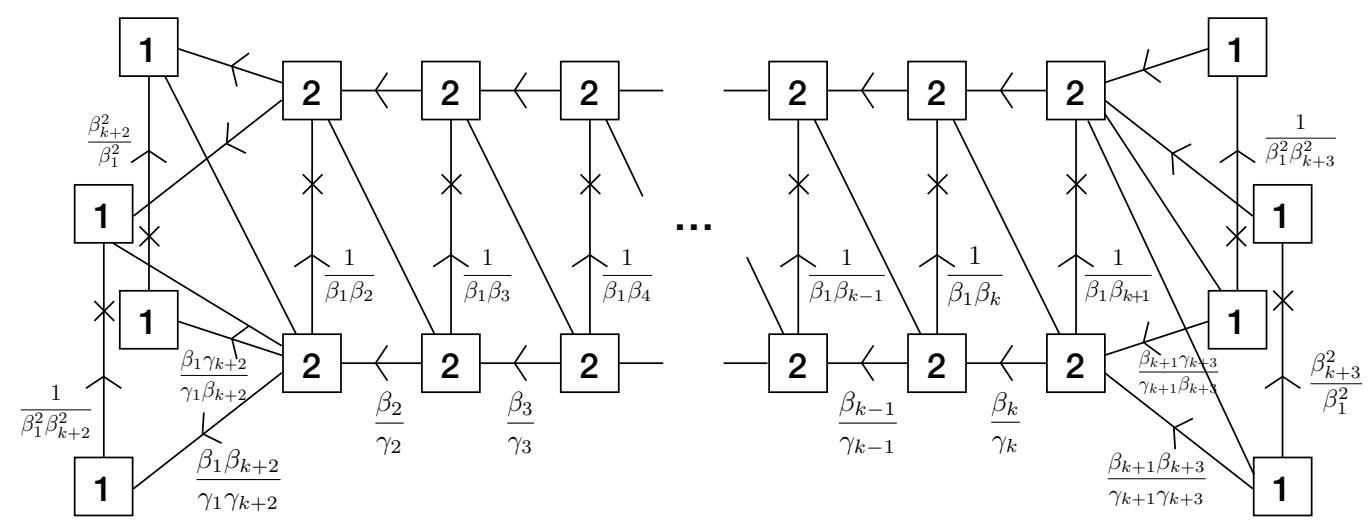

Figure 9. Basic domain wall for $\mathcal{D}=\{+,+, \cdots,+\}_{\beta}$ in the affine $D_{k+3}$ quiver theory. The boxes with 1 denote the $5 \mathrm{~d} \mathrm{SU}(N)_{i=1,2,3,4}$ gauge nodes and the boxes with 2 denote the $5 \mathrm{~d} \mathrm{SU}(2 N)_{i=1, \cdots, k}$ gauge nodes.

a sign $s_{i}= \pm$. Thus the boundary condition of the $5 \mathrm{~d}$ theory at the interface is defined by a vector $\mathcal{B}=\left\{s_{1}, s_{2}, \cdots, s_{k+3}\right\}$ with $s_{i}= \pm$. Basic domain walls have the same boundary condition for two $5 \mathrm{~d}$ theories on both sides.

At the interface, we introduce additional $4 \mathrm{~d}$ chiral multiplets $q_{i}$ and $\tilde{q}_{i}$ coupled to the $5 \mathrm{~d}$ boundary conditions through cubic superpotentials. Like the A-type cases, the $4 \mathrm{~d}$ chiral field $q_{i}$ is a bifundamental field between $H_{i} \times H_{i}^{\prime}$, where $H_{i}$ and $H_{i}^{\prime}$ represent the $i$-th gauge group in the quiver diagram in the first and the second chamber respectively, and $\tilde{q}_{i}$ is a bifundamental field between either $H_{i} \times H_{i+1}^{\prime}$ or $H_{i+1} \times H_{i}^{\prime}$ which is determined by the $4 \mathrm{~d}$ cubic superpotentials. For a given boundary condition $\mathcal{B}$, we can construct two types of basic domain walls, which we call as $\mathcal{T}=\beta$ and $\mathcal{T}=\gamma$, related to the fluxes on $\mathrm{SO}(2 k+6)_{\beta}$ and the fluxes on $\mathrm{SO}(2 k+6)_{\gamma}$ respectively.

Let us first consider the basic domain walls for $\mathrm{SO}(2 k+6)_{\beta}$ fluxes. The simplest boundary condition is $\mathcal{B}=\left\{+^{k+3}\right\}$ where all $X_{i}$ in $\Phi_{i}$ satisfy Neumann boundary condition. In this case we propose a basic domain wall as dipicted in figure 9 . The $5 \mathrm{~d}$ chiral fields $X_{i}$ with Neumann boundary condition in the bottom (or first) chamber are denoted by the horizontal arrows in the bottom forming an affine $D_{k+3}$ diagram with boxes of 1 and 2 . Similarly, the horizontal arrows in the top forming another affine $D_{k+3}$ diagram correspond to another 5 d chiral multiplets $X_{i}^{\prime}$ with Neumann boundary condition in the top (or second) chamber. These $5 \mathrm{~d}$ chiral multiplets $X_{i}$ and $X_{i}^{\prime}$ couple to additional $4 \mathrm{~d}$ chiral multiplets $q$ and $\tilde{q}$ living at the interface represented by the vertical arrows and diagonal arrows, respectively, connecting the top and the bottom affine quiver diagrams. There is a cubic superpotential for each triangle in the figure 9. Also, the baryonic operators from the $4 \mathrm{~d}$ chiral fields $q$ couple to the flip fields denoted by $\times$.

The system with a basic domain wall inserted between two $5 \mathrm{~d}$ affine $D_{k+3}$ quiver theories with boundary condition $\mathcal{B}=\left\{+^{k+3}\right\}$ is consistent in a sense that it has no gauge anomalies. We will show this now. First, we compute the anomaly inflows toward the $4 \mathrm{~d}$ interface from the boundary conditions of the $5 \mathrm{~d}$ theory. As we discussed above, there are two inflow contributions: one from the 5d Yang-Mills terms and another one from the 
matter multiplets with Neumann boundary condition. We first compute the contributions from the YMs terms. The Cartans of the $\mathrm{SO}(2 k+6)_{\beta} \times \mathrm{SO}(2 k+6)_{\gamma}$ symmetry are related to the instanton and the baryon charges as given in (3.7) with the affine $D_{k+3}$ Cartan matrix $A_{i j}$. This relation and the charge assignment for the hypermultiplets in (3.24) tells us that the gauge kinetic terms induce anomaly inflows given by

$$
\begin{array}{rrrl}
\operatorname{Tr}\left(\mathrm{U}(1)_{\beta_{1}, \gamma_{1}} \mathrm{SU}(N)_{1,2}^{2}\right)=\frac{N}{2}, & \operatorname{Tr}\left(\mathrm{U}(1)_{\beta_{1}, \gamma_{1}} \mathrm{SU}(2 N)_{1}^{2}\right)=-\frac{N}{2}, \\
\operatorname{Tr}\left(\mathrm{U}(1)_{\beta_{k+2}, \gamma_{k+2}} \mathrm{SU}(N)_{1}^{2}\right)=\frac{N}{2}, & \operatorname{Tr}\left(\mathrm{U}(1)_{\beta_{k+2}, \gamma_{k+2}} \mathrm{SU}(N)_{2}^{2}\right)=-\frac{N}{2} \\
\operatorname{Tr}\left(\mathrm{U}(1)_{\beta_{i}, \gamma_{i}} \mathrm{SU}(2 N)_{i-1}^{2}\right)=\frac{N}{2}, & \operatorname{Tr}\left(\mathrm{U}(1)_{\beta_{i}, \gamma_{i}} \mathrm{SU}(2 N)_{i}^{2}\right)=-\frac{N}{2}, \\
\operatorname{Tr}\left(\mathrm{U}(1)_{\beta_{k+1}, \gamma_{k+1}} \mathrm{SU}(N)_{3,4}^{2}\right)=-\frac{N}{2}, & \operatorname{Tr}\left(\mathrm{U}(1)_{\beta_{k+1}, \gamma_{k+1}} \mathrm{SU}(2 N)_{k}^{2}\right)=\frac{N}{2}, \\
\operatorname{Tr}\left(\mathrm{U}(1)_{\beta_{k+3}, \gamma_{k+3}} \mathrm{SU}(N)_{3}^{2}\right)=-\frac{N}{2}, & \operatorname{Tr}\left(\mathrm{U}(1)_{\beta_{k+3}, \gamma_{k+3}} \mathrm{SU}(N)_{4}^{2}\right)=\frac{N}{2},
\end{array}
$$

with $2 \leq i \leq k$. There are also matter contributions to the anomaly inflows. The vector multiplets with Neumann boundary condition contribute to the anomaly inflow as

$$
\begin{aligned}
\operatorname{Tr}\left(\mathrm{U}(1)_{R}\right)=\operatorname{Tr}\left(\mathrm{U}(1)_{R}^{3}\right) & =2(k+1) N^{2}-k / 2-2, \\
\operatorname{Tr}\left(\mathrm{U}(1)_{R} \mathrm{SU}(N)_{1,2,3,4}^{2}\right) & =\frac{N}{2}, \quad \operatorname{Tr}\left(\mathrm{U}(1)_{R} \mathrm{SU}(2 N)_{i}^{2}\right)=N \quad \text { for } \quad 1 \leq i \leq k .
\end{aligned}
$$

The hypermultiplet contributions depend on the boundary condition $\mathcal{B}$. For the boundary condition $\mathcal{B}=\left\{+^{k+3}\right\}$, the anomaly inflow contributions from the hypermultiplets are given by

$$
\begin{aligned}
\operatorname{Tr}\left(\mathrm{U}(1)_{\beta_{i}}\right) & =N^{2}, \quad \operatorname{Tr}\left(\mathrm{U}(1)_{\gamma_{i}}\right)=-N^{2} \quad \text { for } \quad 1 \leq i \leq k+1, \\
\operatorname{Tr}\left(\mathrm{U}(1)_{\beta_{1}, \beta_{k+2}} \mathrm{SU}(N)_{1}^{2}\right) & =-\operatorname{Tr}\left(\mathrm{U}(1)_{\gamma_{1}, \gamma_{k+2}} \mathrm{SU}(N)_{1}^{2}\right)=\frac{N}{4}, \\
\operatorname{Tr}\left(\mathrm{U}(1)_{\beta_{1}, \gamma_{k+2}} \mathrm{SU}(N)_{2}^{2}\right) & =-\operatorname{Tr}\left(\mathrm{U}(1)_{\gamma_{1}, \beta_{k+2}} \mathrm{SU}(N)_{2}^{2}\right)=\frac{N}{4}, \\
\operatorname{Tr}\left(\mathrm{U}(1)_{\beta_{i}, \beta_{i+1}} \mathrm{SU}(2 N)_{i}^{2}\right) & =-\operatorname{Tr}\left(\mathrm{U}(1)_{\gamma_{i}, \gamma_{i+1}} \mathrm{SU}(2 N)_{i}^{2}\right)=\frac{N}{4} \quad \text { for } \quad 1 \leq i \leq k, \\
\operatorname{Tr}\left(\mathrm{U}(1)_{\beta_{k+1}, \beta_{k+3}} \mathrm{SU}(N)_{3}^{2}\right) & =-\operatorname{Tr}\left(\mathrm{U}(1)_{\gamma_{k+1}, \gamma_{k+3}} \mathrm{SU}(N)_{3}^{2}\right)=\frac{N}{4}, \\
\operatorname{Tr}\left(\mathrm{U}(1)_{\beta_{k+1}, \gamma_{k+3}} \mathrm{SU}(N)_{4}^{2}\right) & =-\operatorname{Tr}\left(\mathrm{U}(1)_{\gamma_{k+1}, \beta_{k+3}} \mathrm{SU}(N)_{4}^{2}\right)=\frac{N}{4}, \\
\operatorname{Tr}\left(\mathrm{U}(1)_{a} \mathrm{U}(1)_{b} \mathrm{U}(1)_{c}\right) & =\sum_{i} \frac{Q_{a, i} Q_{b, i} Q_{c, i} n_{i}}{2}, \quad \operatorname{Tr}\left(\mathrm{SU}(N)_{1,2}^{3}\right)=N, \quad \operatorname{Tr}\left(\mathrm{SU}(N)_{3,4}^{3}\right)=-N,
\end{aligned}
$$

where $Q_{a, i}$ and $n_{i}$ denote the $\mathrm{U}(1)_{a}$ flavor charge and the number of the $i$-th hypermultiplet respectively. The total anomaly inflows from the $5 \mathrm{~d}$ theory with the boundary condition $\mathcal{B}=\left\{+^{k+3}\right\}$ are sum of these three contributions in (3.25), (3.26), (3.27). Anomaly inflows for other cases with different boundary conditions can be computed in the same way.

We shall check the gauge anomaly cancellation at the interface. There are cubic gauge anomalies coming from the anomaly inflow in (3.27) and they cancel out beautifully by the 

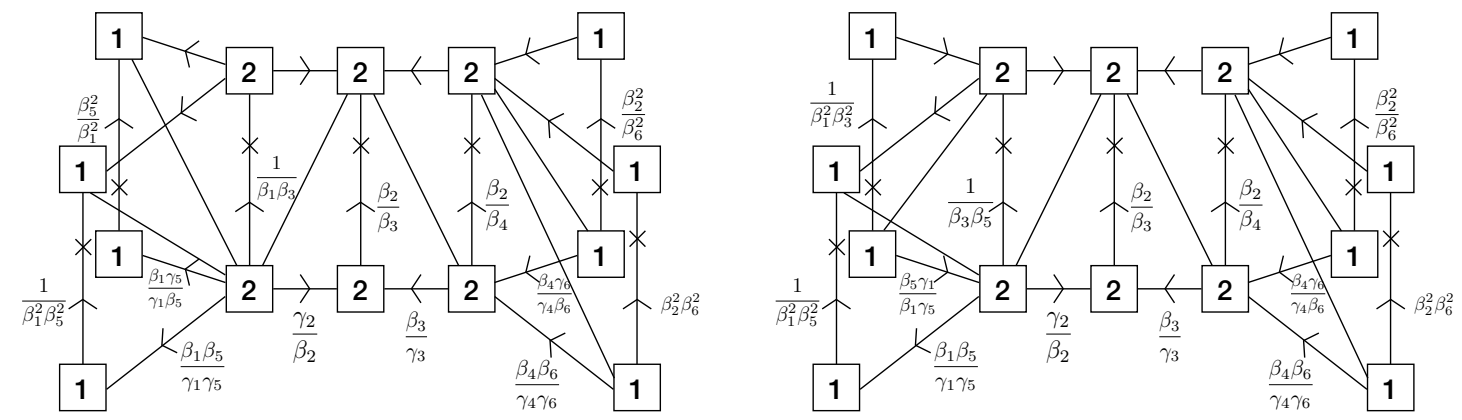

Figure 10. Basic domain walls in the affine $D_{6}$ quiver theory. The left wall is for $\mathcal{D}_{1}=\{++\mid-$ $+\mid++\}_{\beta}$ and the right wall is for $\mathcal{D}_{2}=\{+-|-+|++\}_{\beta}$.

cubic anomalies from the $4 \mathrm{~d}$ chiral multiplets $q$ and $\tilde{q}$ given in figure 9 . Also, the gaugeglobal anomaly cancellation for the $6 \mathrm{~d}$ global symmetries as well as the conditions from the $4 \mathrm{~d}$ superpotentials uniquely fix all the charges of the additional $4 \mathrm{~d}$ degrees of freedom inserted at the interface which we find as drawn in figure 9. For convenience, we scaled the fugacities as $\beta_{i} \rightarrow \beta_{i}^{2}$ and $\gamma_{i} \rightarrow \gamma_{i}^{2}$ in the quiver diagrams for D-type domain walls in this section. This domain wall configuration is thus consistent with no gauge anomaly and no additional global symmetry.

As a consequence, we constructed a consistent domain wall configuration interpolating two 5 d affine $D_{k+3}$ quiver gauge theories. Note that the $\mathrm{U}(1)_{\beta_{1}}, \mathrm{U}(1)_{\beta_{2}}, \cdots, \mathrm{U}(1)_{\beta_{k+1}}$ symmetries are cyclically permuted, and $\mathrm{U}(1)_{\beta_{k+2}}$ and $\mathrm{U}(1)_{\beta_{k+3}}$ symmetries are flipped as we move across the domain wall. Namely,

$$
\sigma\left(\beta_{1}, \beta_{2}, \cdots, \beta_{k}, \beta_{k+1}, \beta_{k+2}, \beta_{k+3}\right) \rightarrow\left(\beta_{2}, \beta_{3}, \cdots, \beta_{k+1}, \beta_{1}, 1 / \beta_{k+2}, 1 / \beta_{k+3}\right),
$$

in terms of fugacities $\beta_{i}$. All other basic domain walls for other $\mathcal{D}_{\mathcal{T}}$ can be similarly constructed.

For example, two other basic domain walls in the affine $D_{6}$ quiver gauge theory are given in figure 10. The left quiver diagram corresponds to the basic domain wall for $\mathcal{D}_{1}=\{++|-+|++\}_{\beta}$ where the first two and the last two signs denote the boundary conditions for the $\mathrm{SU}(N)_{1,2} \times \mathrm{SU}(2 N)_{1}$ and the $\mathrm{SU}(N)_{3,4} \times \mathrm{SU}(2 N)_{3}$ bifundamental hypers respectively. The $\mathrm{U}(1)_{\beta}$ global symmetries are permuted by this domain wall as

$$
\sigma\left(\beta_{1}, \beta_{2}, \beta_{3}, \beta_{4}, \beta_{5}, \beta_{6}\right) \rightarrow\left(\beta_{3}, 1 / \beta_{1}, \beta_{4}, 1 / \beta_{2}, 1 / \beta_{5}, 1 / \beta_{6}\right),
$$

in terms of the fugacities $\beta_{i}$ for the $\mathrm{U}(1)_{\beta_{i}}$. On the other hand, the right quiver diagram corresponds to the domain wall for $\mathcal{D}_{2}=\{+-|-+|++\}_{\beta}$ and it permutes the $\mathrm{U}(1)_{\beta}$ symmetries as

$$
\sigma\left(\beta_{1}, \beta_{2}, \beta_{3}, \beta_{4}, \beta_{5}, \beta_{6}\right) \rightarrow\left(1 / \beta_{1}, 1 / \beta_{5}, \beta_{4}, 1 / \beta_{2}, \beta_{3}, 1 / \beta_{6}\right) .
$$

Another example is depicted in figure 11. This domain wall is for $\mathcal{D}=\{+,+, \cdots,+\}_{\gamma}$ associated to the flux on $\mathrm{SO}(2 k+6)_{\gamma}$.

We will now relate the domain walls constructed by connecting multiple basic domain walls with fluxes in the $6 \mathrm{~d}\left(D_{k+3}, D_{k+3}\right)$ conformal matter theory. We first need to identify 

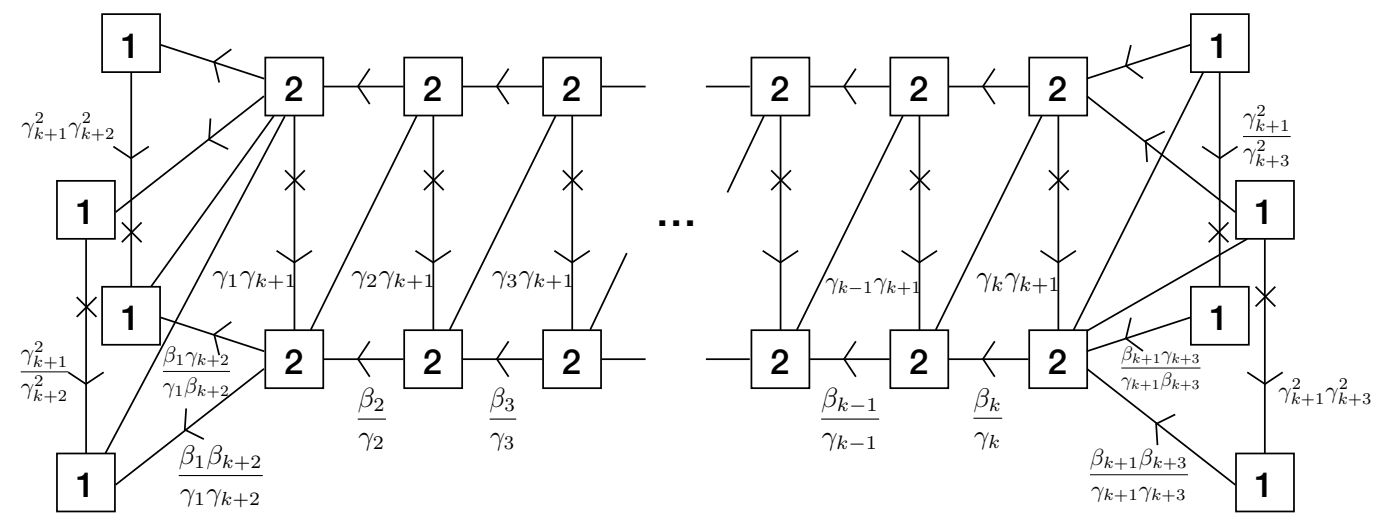

Figure 11. Basic domain wall for $\mathcal{D}=\{+,+, \cdots,+\}_{\gamma}$ in the affine $D_{k+3}$ quiver theory.

fluxes for the basic domain walls. We will employ the flux assignement given in (3.14). We find that the flux $Q_{i}$ on a $\mathrm{U}(1)_{i}$ global symmetry is given by

$$
Q_{i}=\frac{1}{h_{D_{2 k+6}}^{\vee} N} \sum_{f} q_{i, f}=\frac{1}{(2 k+4) N} \sum_{f} q_{i, f},
$$

where $q_{i, f}$ denotes the $\mathrm{U}(1)_{i}$ charge for the $f$-th flip field. For instance, the basic domain wall of $\mathcal{D}=\left\{+^{k+3}\right\}_{\beta}$ drawn in figure 9 corresponds to the flux $\left(\frac{k+4}{2 k+4},\left(\frac{1}{2 k+4}\right)^{k}, 0,0\right)_{\beta}$ in $\mathrm{SO}(2 k+6)_{\beta}$ symmetry. Similarly, the two basic domain walls in figure 10 for $\mathcal{D}=$ $\{++|-+|++\}_{\beta}$ and $\mathcal{D}=\{+-|-+|++\}_{\beta}$ correspond to the fluxes $\frac{1}{10}(3,-4,2,1,0,0)_{\beta}$ and $\frac{1}{10}(2,-4,3,1,2,0)_{\beta}$, respectively, in $\mathrm{SO}(12)_{\beta}$. When we join multiple basic domain walls, the total flux $F_{\text {tot }}$ of the final domain wall configuration is simply the sum of the fluxes on all basic domain walls.

For the D-type flux domain walls, we will propose Conjectures in section 3.1 with $G=D_{k+3}$. The simplest exercise is to combine $k+1$ (or $2 k+2$ ) basic domain walls of the same type for odd $k$ (or even $k$ ). When we put this 5 d domain wall configuration on a finite interval, it corresponds to the $6 \mathrm{~d}$ theory on a tube with integer fluxes. For example, we can consider the $5 \mathrm{~d}$ theory on an interval with $k+1$ copies of the basic domain wall of $\mathcal{D}=\{+,+, \cdots,+\}_{\beta}$ drawn in figure 9 which gives rise to an integer flux $F_{\text {tot }}=\left(1^{k+1}, 0,0\right)_{\beta}$ for odd $k$. Choosing the maximal boundary condition, this theory reduces to a $4 \mathrm{~d} \mathcal{N}=1$ quiver gauge theory at low energy. We claim this $4 \mathrm{~d}$ theory realizes the $6 \mathrm{~d}\left(D_{k+3}, D_{k+3}\right)$ conformal matter theory on a tube with flux $F_{\text {tot }}=\left(1^{k+1}, 0,0\right)_{\beta}$ and maximal punctures at the two ends. When $k$ is even, we can combine $2 k+2$ basic domain walls of type $\mathcal{D}=\{+,+, \cdots,+\}_{\beta}$, and this theory on an interval gives rise to the 4 d quiver theory corresponding to the $6 \mathrm{~d}$ theory on a tube with flux $F_{\text {tot }}=\left(2^{k+1}, 0,0\right)_{\beta}$. We have checked for several $k$ 's that the 't Hooft anomalies of the $4 \mathrm{~d}$ quiver theory perfectly agree with those from the $6 \mathrm{~d}$ anomaly polynomial and anomaly inflow at the two punctures.

Similarly, when we combine 4 copies of the basic domain walls in figure 10 on a tube, we will obtain the $4 \mathrm{~d}$ quiver gauge theories corresponding to the $6 \mathrm{~d}$ conformal matter theory on a tube with fluxes $F=(1,-1,1,1,0,0)_{\beta}$ for the left type and $F=(0,-1,1,1,1,0)_{\beta}$ for 


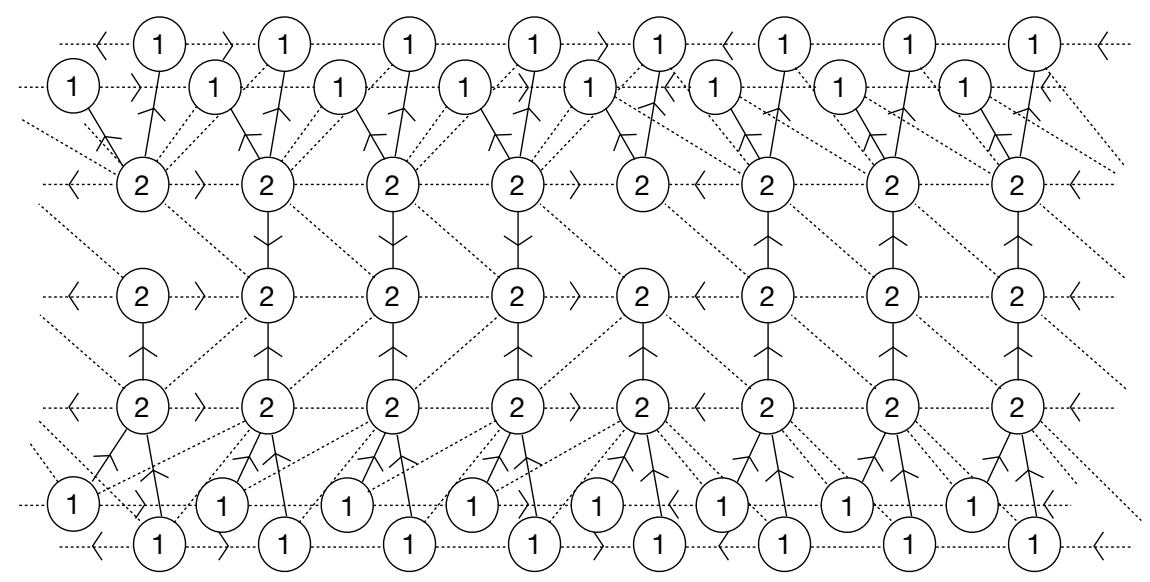

Figure 12. Flux domain wall configuration with flux $F_{\text {tot }}=(1,-1,1,1,0,0)_{\beta}+(1,1,1,1,0,0)_{\gamma}$ in the affine $D_{6}$ quiver theory on a circle. The horizontal direction is the circle direction and both ends are identified. The $4 \mathrm{~d}$ chiral fields are denoted by dashed lines. There is a flip field on each horizontal arrow.

the right type. We checked these theories by comparing their 't Hooft anomalies against expected anomalies from the $6 \mathrm{~d}$ theory.

We can also consider domain wall configurations on a circle which realize the $6 \mathrm{~d}$ conformal matter theories on a torus with flux. The simplest example is to glue two ends of the tube theory from the $k+1$ copies of the basic domain wall in figure 9. Indeed, the resulting $4 \mathrm{~d}$ theory has the expect 't Hooft anomalies for the torus theory with flux $F=\left(1^{k+1}, 0,0\right)_{\beta}$.

More general flux domain walls can be constructed by considering more complicated combinations of basic domain walls. An example is given in figure 12. Here, we combined four copies of a domain wall with $F_{1}=\frac{1}{10}(3,-4,2,1,0,0)_{\beta}$ and another four copies of a domain wall with $F_{2}=\frac{1}{10}(6,1,1,1,0,0)_{\gamma}$. So the total flux of the final domain wall configuration is $F_{\text {tot }}=(1,-1,1,1,0,0)_{\beta}+(1,1,1,1,0,0)_{\gamma}$. When we compactify this $5 \mathrm{~d}$ theory with domain walls on a circle, we will obtain at low energy the $4 \mathrm{~d}$ quiver theory given in figure 12 . This $4 \mathrm{~d}$ theory corresponds to the $6 \mathrm{~d}\left(D_{6}, D_{6}\right)$ conformal matter theory on a torus with flux $F_{\text {tot }}$. We have checked that this $4 \mathrm{~d}$ theory has the correct 't Hooft anomalies for being the torus theory with flux $F_{\text {tot }}$.

Let us now discuss the domain walls for the minimal $\left(D_{k+3}, D_{k+3}\right)$ conformal matter theories with fluxes. The construction of the flux domain wall in this theory is almost parallel to that in the non-minimal conformal matter theory with $N>1$. The $5 \mathrm{~d}$ theory is a linear quiver gauge theory with $\mathrm{SU}(2)^{k}$ gauge groups and the first and last gauge nodes have 4 fundamental hypermultiplets. As explained already, the basic domain walls can be constructed by $4 \mathrm{~d}$ interfaces with $4 \mathrm{~d}$ chiral multiplets $q_{i}, \tilde{q}_{i}$ and flip fields coupled to $5 \mathrm{~d}$ boundary conditions on both sides through cubic superpotentials. The new feature of the minimal conformal matter here is that the symmetry is enhanced to $D_{2 k+6}$ (and for $k=1$ to $E_{8}$ ). This means that we have a larger Weyl symmetry, and thus domain walls can be engineered which manifest this by permuting $\gamma$ and $\beta$ symmetries. This is related to 
the fact that in the end of the quiver the $\mathrm{SU}(1)$ symmetries are empty which gives rise to combining the $\mathrm{U}(1)$ symmetries under which bifundamentals at the end of the quivers are charged into two SU(4) global symmetries. We can write the domain wall as in figure 9 or as figure 13. Note that these differ by choices of boundary conditions. The latter exists only for the minimal case and is more natural here so we will use it. However, the procedure of reading off the fluxes from the flip fields only applies to the former.

The $5 \mathrm{~d}$ boundary condition is labeled by a sign vector $\mathcal{B}=\left\{s_{1}, \cdots, s_{4}\left|s_{5}, \cdots, s_{k+3}\right|\right.$ $\left.s_{k+4}, \cdots, s_{k+7}\right\}$ where the $i$-th element $s_{i}$ denotes the boundary condition of the $i$-th hypermultiplet. In this $\mathcal{B}$, the first four elements are for the fundamental hypermultiplets of the first gauge node and the last four are for the fundamentals of the last gauge node. This condition breaks the $\mathrm{SO}(8) \times \mathrm{SO}(8)$ global symmetries rotating the fundamental flavors at the ends of the quiver to $\mathrm{U}(1)^{2} \times \mathrm{SU}(4) \times \mathrm{SU}(4)$ symmetry. At the interface, the chiral halves of $k-1$ bifundamental hypermultiplets, chosen by $s_{i}$, satisfy Neumann boundary conditions and they couple to the $4 \mathrm{~d}$ chiral fields $q_{i}$ and $\tilde{q}_{i}$ through cubic superpotentials, which we have seen in the non-minimal cases. Note that, since the gauge groups are now all pseudo-real, we can freely choose the chiral field $\tilde{q}_{i}$ to be in either $\mathrm{SU}(2)_{i} \times \mathrm{SU}(2)_{i+1}^{\prime}$ or $\mathrm{SU}(2)_{i+1} \times \mathrm{SU}(2)_{i}^{\prime}$ and these choices yield different domain walls. Also, we will introduce new cubic superpotentials at the interface for the chiral halves $M_{i}$ and $M_{i}^{\prime}$ of the fundamental hypers at the two ends of the quiver as $\tilde{\mathcal{W}}=\sum_{i=1}^{4} M_{i} q_{1} M_{i}^{\prime}+\sum_{i=k+4}^{k+7} M_{i} q_{k} M_{i}^{\prime}$. This identifies the boundary conditions as $s_{i}=-s_{i}$ for $i=1, \cdots, 4, k+4, \cdots, k+7$.

Since the gauge groups are $\mathrm{SU}(2)$, cubic gauge anomalies are absent at the interface. The gauge-global mixed anomalies from the boundary conditions of the $5 \mathrm{~d}$ theory in the first chamber are the following. First, the anomaly inflows from the Yang-Mills kinetic terms are given by

$$
\begin{gathered}
\operatorname{Tr}\left(\mathrm{U}(1)_{\beta_{i}} \mathrm{SU}(2)_{i-1}^{2}\right)=\operatorname{Tr}\left(\mathrm{U}(1)_{\gamma_{i}} \mathrm{SU}(2)_{i-1}^{2}\right)=\frac{1}{2}, \\
\operatorname{Tr}\left(\mathrm{U}(1)_{\beta_{i}} \mathrm{SU}(2)_{i}^{2}\right)=\operatorname{Tr}\left(\mathrm{U}(1)_{\gamma_{i}} \mathrm{SU}(2)_{i}^{2}\right)=-\frac{1}{2},
\end{gathered}
$$

with $2 \leq i \leq k$. Here, $\prod_{i=1}^{k+1} \mathrm{U}(1)_{\beta_{i}} \times \prod_{i=2}^{k} \mathrm{U}(1)_{\gamma_{i}}$ are the abelian global symmetries of the $5 \mathrm{~d}$ theory. Then the hypermultiplets with $\mathcal{B}=\left\{+^{k+7}\right\}$ induce the inflow contributions as

$$
\begin{gathered}
\operatorname{Tr}\left(\mathrm{U}(1)_{\beta_{1}} \mathrm{SU}(2)_{1}^{2}\right)=\operatorname{Tr}\left(\mathrm{U}(1)_{\beta_{k+1}} \mathrm{SU}(2)_{k}^{2}\right)=\frac{1}{2}, \\
\operatorname{Tr}\left(\mathrm{U}(1)_{\beta_{i}} \mathrm{SU}(2)_{i-1}^{2}\right)=\operatorname{Tr}\left(\mathrm{U}(1)_{\beta_{i}} \mathrm{SU}(2)_{i}^{2}\right)=\frac{1}{4}, \\
\operatorname{Tr}\left(\mathrm{U}(1)_{\gamma_{i}} \mathrm{SU}(2)_{i-1}^{2}\right)=\operatorname{Tr}\left(\mathrm{U}(1)_{\gamma_{i}} \mathrm{SU}(2)_{i}^{2}\right)=-\frac{1}{4},
\end{gathered}
$$

with $2 \leq i \leq k$. The requirement of these anomaly cancellation uniquely fixes all flavor charges of the $4 \mathrm{~d}$ chiral fields. Also, the $4 \mathrm{~d}$ fields $q_{i}$ and $\tilde{q}_{i}$ should have R-charges 0 and +1 to cancel the gauge- $\mathrm{R}$ mixed anomaly from the $5 \mathrm{~d}$ vector multiplets. The same is true for the other boundary conditions $\mathcal{B}$. So these basic domain walls can be consistently inserted into the $5 \mathrm{~d}$ system.

Three different basic domain walls in the affine $D_{8}$ quiver theory are depicted in figure 13. The fluxes for these domain walls can be determined from the linear anomalies 

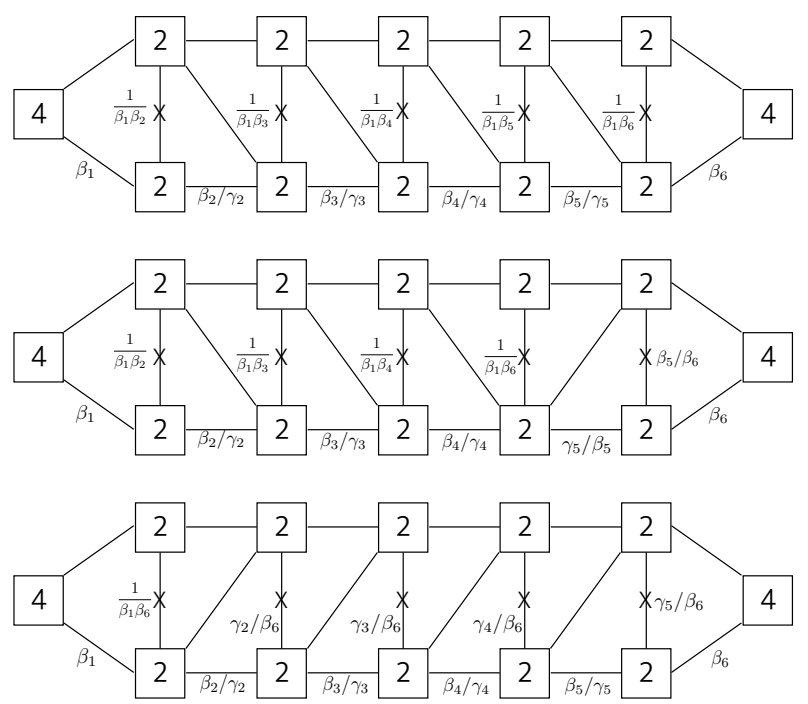

Figure 13. Basic domain walls with the boundary conditions $\mathcal{B}_{1}=\left\{+^{4}\left|+{ }^{4}\right|+{ }^{4}\right\}$ and $\mathcal{B}_{2}=$ $\left\{+{ }^{4}\left|+{ }^{3},-\right|+{ }^{4}\right\}$ and $\mathcal{B}_{3}=\left\{+{ }^{4}\left|+{ }^{4}\right|+{ }^{4}\right\}$, respectively, in the first chamber. The first and the third domain walls have the same boundary condition in the first chamber, but they have different $4 \mathrm{~d}$ chiral fields $q_{i}$ and superpotentials.

as we have outlined previously. The corresponding fluxes are

$$
\begin{aligned}
& F_{1}=\frac{1}{14}(7,2,1,1,1,2,0,0,0,0), \\
& F_{2}=\frac{1}{14}(5,2,1,1,-2,3,0,0,0,0), \\
& F_{3}=\frac{1}{14}(2,0,0,0,0,7,-1,-1,-1,-2),
\end{aligned}
$$

in the $\mathrm{U}(1)_{\beta}^{6} \times \mathrm{U}(1)_{\gamma}^{4}$ abelian symmetries for the three domain walls respectively. For all these cases, the fluxes for $\beta_{7}, \beta_{8}, \gamma_{1}$, and $\gamma_{6-8}$ are zero and we have not written them for brevity. These domain walls permute the flavor symmetries with non-zero fluxes as follows:

$$
\begin{aligned}
& \sigma\left(\beta_{1}, \beta_{2}, \beta_{3}, \beta_{4}, \beta_{5}, \beta_{6}\right) \rightarrow\left(\beta_{2}, \beta_{3}, \beta_{4}, \beta_{5}, \beta_{6}, \beta_{1}\right), \\
& \sigma\left(\beta_{1}, \beta_{2}, \beta_{3}, \beta_{4}, \beta_{5}, \beta_{6}\right) \rightarrow\left(\beta_{2}, \beta_{3}, \beta_{4}, \beta_{6},-\beta_{1},-\beta_{5}\right), \\
& \sigma\left(\beta_{1}, \beta_{6}, \gamma_{2}, \gamma_{3}, \gamma_{4}, \gamma_{5}\right) \rightarrow\left(\beta_{6},-\gamma_{5},-\beta_{1}, \gamma_{2}, \gamma_{3}, \gamma_{4}\right) .
\end{aligned}
$$

We claim that the Conjectures above hold for the flux domain walls constructed by joining these basic domain walls in the minimal D-type conformal matter theories. For example, we can connect 6 copies of the first domain wall in figure 13 and then put this $5 \mathrm{~d}$ theory on an interval with maximal boundary conditions at the two ends. Then we conjecture that the resulting $4 \mathrm{~d}$ quiver gauge theory at low energy corresponds to the minimal $\left(D_{8}, D_{8}\right)$ conformal matter theory with flux $F_{\text {tot }}=\left(1^{6}, 0^{4}\right)$ on a tube with two maximal punctures. Indeed, the 't Hooft anomalies of this $4 \mathrm{~d}$ quiver theory agree with the expected results obtained from the $6 \mathrm{~d}$ anomaly polynomial on the tube together with the inflow contributions at the two punctures. We will see more examples for these conjectures for tube theories and torus theories in the next section. 

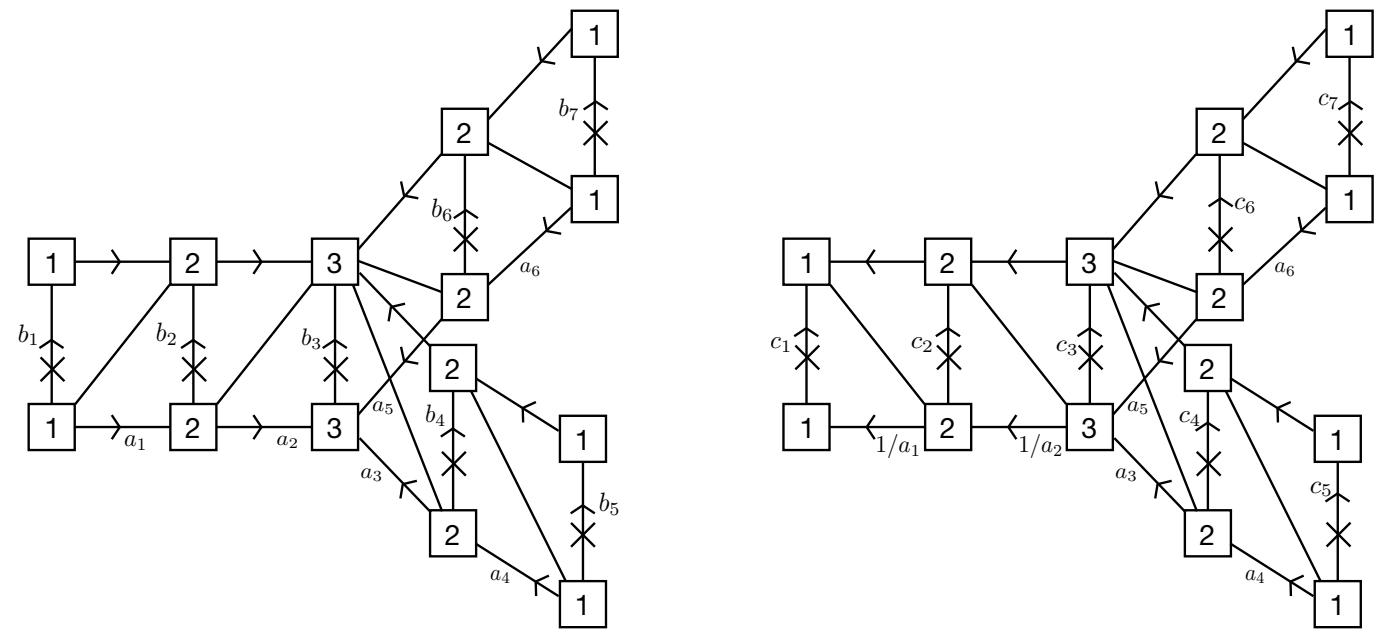

Figure 14. Basic domain walls in the affine $E_{6}$ quiver theory with $\mathcal{D}_{1}=\left\{+^{6}\right\}_{\beta}$ and $\mathcal{D}_{2}=$ $\left\{-,-,+{ }^{4}\right\}_{\beta}$, respectively. The integer numbers $l_{i}$ in the boxes denote the $\mathrm{SU}\left(l_{i} N\right)$ gauge symmetries at the interface.

\subsection{E-type domain walls}

Now we turn to the flux domain walls in the $5 \mathrm{~d}$ affine E-type quiver gauge theories. We can construct these domain walls by applying the same idea used for the A- and D-type domain wall systems presented in the previous subsections.

Let us start by discussing the domain walls in the affine $E_{6}$ quiver gauge theory for general $N$ M5-branes. This quiver theory has $\mathrm{U}(1)^{6}$ flavor symmetry of the bifundamental hypers and $\mathrm{U}(1)^{7}$ instanton symmetry. There are basic domain walls arising from a single interface interpolating between two $5 \mathrm{~d}$ theories with $1 / 2$ BPS boundary conditions given in (3.1) and (3.2). These domain walls are labeled by $\mathcal{D}=\mathcal{B}_{\mathcal{T}}$ where $\mathcal{B}=\left\{s_{1}, s_{2}, \cdots, s_{6}\right\}$ denotes the boundary conditions of six hypermultiplets and $\mathcal{T}$ denotes the representations of the $4 \mathrm{~d}$ chiral fields $q_{i}$. At the interface, we have $\prod_{i=1}^{6} \mathrm{SU}\left(l_{i} N\right) \times \mathrm{SU}\left(l_{i} N\right)^{\prime}$ gauge symmetry with $\left\{l_{i}\right\}=\{1,2,3,2,1,2,1\}$ coming from the 5 d vector multiplets with Neumann boundary condition in two sides. The $4 \mathrm{~d}$ interface includes $4 \mathrm{~d}$ chiral fields $q_{i}$ and $\tilde{q}_{i}$ and the cubic superpotentials of the form in (3.3) whose explicit expressions are fixed by the domain wall data $\mathcal{B}$ and $\mathcal{T}$.

We present two basic domain walls for fluxes on the $\mathrm{U}(1)_{\beta}^{6}$ of the first $E_{6}$ global symmetry in figure 14. The fugacities $a_{i}, b_{i}, c_{i}$ are related to the fugacities $\beta_{i}, \gamma_{i}$ for the Cartans $\prod_{i=1}^{6} \mathrm{U}(1)_{\beta_{i}} \times \mathrm{U}(1)_{\gamma_{i}}$ of $E_{6} \times E_{6}$. Regarding the mass parameters of the baryon symmetries given in (3.8), the fugacities $a_{i}$ for the chiral halves of the 5 d hypermultiplets are given by

$$
\begin{array}{ll}
a_{1}=\left(\beta_{2} \gamma_{1} / \beta_{1} \gamma_{2}\right)^{1 / 2}, \quad a_{2}=\left(\beta_{3}^{2} \gamma_{1} \gamma_{2} / \beta_{1} \beta_{2} \gamma_{3}^{2}\right)^{1 / 6}, & a_{3}=\left(\beta_{1} \beta_{2} \beta_{3} \beta_{5}^{3} \beta_{6} \gamma_{4}^{3} / \beta_{4}^{3} \gamma_{1} \gamma_{2} \gamma_{3} \gamma_{5}^{3} \gamma_{6}\right)^{1 / 12}, \\
a_{4}=\left(\beta_{1} \beta_{2} \beta_{3} \beta_{4} \beta_{6} \gamma_{5} / \beta_{5} \gamma_{1} \gamma_{2} \gamma_{3} \gamma_{4} \gamma_{6}\right)^{1 / 4}, & a_{5}=\left(\beta_{1} \beta_{2} \beta_{3} \gamma_{4}^{3} \gamma_{5}^{3} \gamma_{6} / \beta_{4}^{3} \beta_{5}^{3} \beta_{6} \gamma_{1} \gamma_{2} \gamma_{3}\right)^{1 / 12}, \\
a_{6}=\left(\beta_{1} \beta_{2} \beta_{3} \beta_{4} \beta_{5} \gamma_{6} / \beta_{6} \gamma_{1} \gamma_{2} \gamma_{3} \gamma_{4} \gamma_{5}\right)^{1 / 4} . &
\end{array}
$$

Here, we have chosen the Cartans $\mathrm{U}(1)_{\beta_{i}}$ and $\mathrm{U}(1)_{\gamma_{i}}$ in the orthogonal basis of $E_{6}$ such 
that the fundamentals of $\mathrm{SO}(10) \subset E_{6}$ carry unit charges under these Cartans. Specifically, using the $\mathrm{SO}(10) \times \mathrm{U}(1)$ subgroup of $E_{6}$, the $\beta_{1-5}$ fugacities parametrize the $\mathrm{SO}(10)$ and $\beta_{6}$ parametrize the $\mathrm{U}(1)$, where the latter is normalized such the $\mathbf{1 0}$ of $\mathrm{SO}(10)$, appearing in the decomposition of the $\mathbf{2 7}$, has charge 1 . The $\mathrm{U}(1)_{\gamma_{i}}$ symmetries use the same basis. Also, the anomaly free condition for the Cartans $\mathrm{U}(1)_{\beta_{i}}$ and $\mathrm{U}(1)_{\gamma_{i}}$ and the $4 \mathrm{~d}$ superpotential constraints fully determine all fugacities for the $4 \mathrm{~d}$ chiral fields as

$$
\begin{array}{lll}
b_{1}=\beta_{4} / \beta_{1}, \quad b_{2}=\left(\beta_{4} / \beta_{2}\right)^{1 / 2}, & b_{3}=\left(\beta_{4} / \beta_{3}\right)^{1 / 3}, & b_{4}=\left(\beta_{5} / \beta_{3}\right)^{1 / 2}, \\
b_{5}=\left(\beta_{1} \beta_{2} \beta_{4} \beta_{5} \beta_{6} / \beta_{3}\right)^{1 / 2}, & b_{6}=\left(\beta_{3} \beta_{5}\right)^{-1 / 2}, & b_{7}=\left(\beta_{1} \beta_{2} \beta_{4} / \beta_{3} \beta_{5} \beta_{6}\right)^{1 / 2},
\end{array}
$$

for the first domain wall and

$$
\begin{aligned}
& c_{1}=\beta_{2} / \beta_{1}, \quad c_{2}=\left(\beta_{3} / \beta_{1}\right)^{1 / 2}, \quad c_{3}=\left(\beta_{4} / \beta_{1}\right)^{1 / 3}, \quad c_{4}=\left(\beta_{5} / \beta_{1}\right)^{1 / 2}, \\
& c_{5}=\left(\beta_{2} \beta_{3} \beta_{4} \beta_{5} \beta_{6} / \beta_{1}\right)^{1 / 2}, \quad c_{6}=\left(\beta_{1} \beta_{5}\right)^{-1 / 2}, \quad c_{7}=\left(\beta_{2} \beta_{3} \beta_{4} / \beta_{1} \beta_{5} \beta_{6}\right)^{1 / 2} \text {, }
\end{aligned}
$$

for the second domain wall. One can check that these domain walls, when coupled to the $5 \mathrm{~d}$ boundary conditions, have no cubic gauge anomalies and in total $\mathrm{U}(1)_{\beta}^{6} \times \mathrm{U}(1)_{\gamma}^{6} \subset E_{6} \times E_{6}$ anomaly-free abelian global symmetries. We can construct all the other basic domain walls in the same way by choosing different domain wall data $\mathcal{B}=\left\{s_{i}\right\}$ and $\mathcal{T}=\beta$ or $\gamma$.

The global symmetries are permuted by the domain walls. The first domain wall with $\mathcal{D}_{1}=\left\{+{ }^{6}\right\}_{\beta}$ in figure 14 permutes the $\mathrm{U}(1)_{\beta_{i}}$ symmetries as

$$
\begin{array}{lll}
\beta_{1} \rightarrow\left(\beta_{3} \beta_{4} / \beta_{1} \beta_{2}\right)^{1 / 2}, & \beta_{2} \rightarrow\left(\beta_{1} \beta_{3} / \beta_{2} \beta_{4}\right)^{1 / 2}, & \beta_{3} \rightarrow\left(\beta_{2} \beta_{3} / \beta_{1} \beta_{4}\right)^{1 / 2}, \\
\beta_{4} \rightarrow\left(\beta_{1} \beta_{2} \beta_{3} \beta_{4}\right)^{-1 / 2}, & \beta_{5} \rightarrow\left(\beta_{6} / \beta_{5}\right)^{1 / 2}, & \beta_{6} \rightarrow\left(\beta_{5}^{3} \beta_{6}\right)^{-1 / 2},
\end{array}
$$

in terms of the $\mathrm{U}(1)_{\beta_{i}}$ fugacities, and the second domain wall with $\mathcal{D}_{1}=\left\{-,-,+{ }^{4}\right\}_{\beta}$ permutes the symmetries as

$$
\begin{array}{lll}
\beta_{1} \rightarrow\left(\beta_{1} \beta_{2} / \beta_{3} \beta_{4}\right)^{1 / 2}, & \beta_{2} \rightarrow\left(\beta_{1} \beta_{3} / \beta_{2} \beta_{4}\right)^{1 / 2}, & \beta_{3} \rightarrow\left(\beta_{1} \beta_{4} / \beta_{2} \beta_{3}\right)^{1 / 2}, \\
\beta_{4} \rightarrow\left(\beta_{1} \beta_{2} \beta_{3} \beta_{4}\right)^{-1 / 2}, & \beta_{5} \rightarrow\left(\beta_{6} / \beta_{5}\right)^{1 / 2}, & \beta_{6} \rightarrow\left(\beta_{5}^{3} \beta_{6}\right)^{-1 / 2} .
\end{array}
$$

We propose the Conjectures in section 3.1 hold for the flux domain walls in the affine $E_{6}$ quiver theory engineered by connecting these basic domain walls. The flux assignment for each basic domain wall is given by (3.14) with $h^{\vee}=12$. So the first domain wall in figure 14 corresponds to the $6 \mathrm{~d}$ flux $F=\left(0,0, \frac{1}{3},-\frac{1}{3}, 0,0\right)_{\beta}$ and the second domain wall is mapped to the 6 d flux $F=\left(\frac{1}{2},-\frac{1}{6},-\frac{1}{6},-\frac{1}{6}, 0,0\right)_{\beta}$.

When we connect 6 copies of the first domain wall, the resulting domain wall configuration has flux $F=(0,1,2,-1,0,0)_{\beta}$ with $\prod_{i=1}^{6} \sigma^{t_{i}}=1$ after carefully taking into account the above permutations. This flux is the minimal integral flux breaking $E_{6} \rightarrow$ $\mathrm{SU}(3) \times \mathrm{SU}(3) \times \mathrm{SU}(2) \times \mathrm{U}(1)$. The Conjectures predict that $4 \mathrm{~d}$ reductions of this configuration by putting it on a circle or an interval with maximal boundary conditions give rise to the $6 \mathrm{~d}\left(E_{6}, E_{6}\right)$ conformal matter theory of $N$ M5-branes carrying the same flux $F$ compactified on a torus or a tube with two maximal punctures. Indeed, we checked these $4 \mathrm{~d}$ theories obtained from the $5 \mathrm{~d}$ flux domain walls have the same 't Hooft anomalies as 

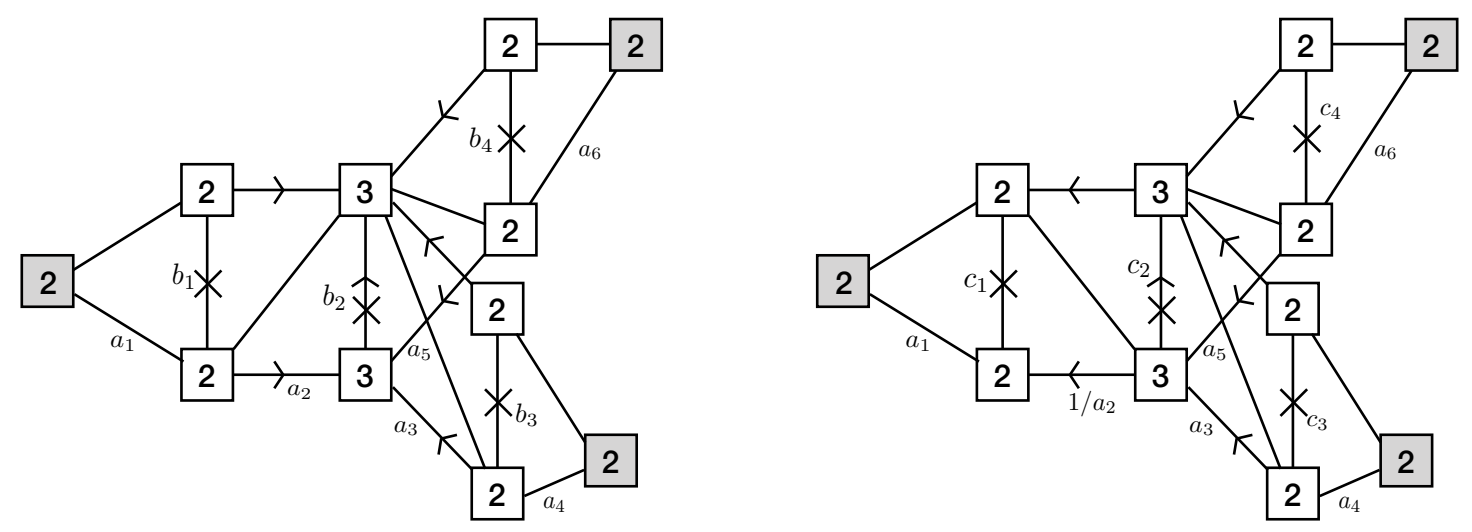

Figure 15. Basic domain walls in the minimal $E_{6}$ theory with $\mathcal{D}_{1}=\left\{+{ }^{6}\right\}_{\beta}$ and $\mathcal{D}_{2}=\left\{+,-,+^{4}\right\}_{\beta}$, respectively. The shaded boxes stand for $\mathrm{SU}(2)^{3}$ flavor nodes in the bulk $5 \mathrm{~d}$ theory.

those computed from the compactification of the $6 \mathrm{~d}$ anomaly polynomial and the anomaly inflows for the maximal punctures. For example, the $4 \mathrm{~d}$ theory on a torus has the central charges as

$$
a=\frac{2 N \sqrt{3}(9 N-4)^{3 / 2}}{\sqrt{N(3 N-1)}}, \quad c=\frac{N(18 N-7) \sqrt{3(9 N-4)}}{\sqrt{N(3 N-1)}},
$$

which are precisely the central charges of the $4 \mathrm{~d}$ quiver theory obtained from the $5 \mathrm{~d}$ theory with domain walls of the flux $F=(0,1,2,-1,0,0)_{\beta}$ on a circle. We have performed similar computations using other combinations of basic domain walls and the results agree with our conjectures. Note that the six dimensional computation is agnostic about some of the fields becoming free and thus for comparison we do not decouple the free fields. The above values are not the superconformal anomalies as we do not take into account the accidental $\mathrm{U}(1)$ symmetries coming from free fields. As we claim to identify the symmetries correctly in six and four dimensions, the above computation is a simple non-trivial check of matching 't Hooft anomalies between the six dimensional and four dimensional computations.

The flux domain walls for the minimal $E_{6}$ conformal matter theory when $N=1$ can be constructed as follows. The $5 \mathrm{~d}$ theory is a quiver gauge theory of $\mathrm{SU}(2)^{3} \times \mathrm{SU}(3)$ gauge groups with two fundamental hypers for each $\mathrm{SU}(2)$ gauge node. Let us define a basic domain wall labeled by $\mathcal{D}=\mathcal{B}_{\mathcal{T}}$ as a $4 \mathrm{~d}$ interface connecting two $5 \mathrm{~d}$ quiver theories with boundary condition $\mathcal{B}=\left\{s_{1}, s_{2}, \cdots, s_{6}\right\}$. Here we assume that two $\mathrm{SU}(2)$ fundamental hypermultiplets for an $\mathrm{SU}(2)$ gauge node have the same boundary conditions for $\mathcal{T}=\beta$ and the opposite boundary conditions for $\mathcal{T}=\gamma$. The domain wall adds four $4 \mathrm{~d}$ chiral multiplets $q_{i}$ and $\tilde{q}_{i}$ as explained. The index $\mathcal{T}=\beta$ or $\gamma$ denotes the representation of the $4 \mathrm{~d}$ field $q_{4}$ either in $(\overline{\mathbf{3}}, \mathbf{3})$ or $(\mathbf{3}, \overline{\mathbf{3}})$ of the $\mathrm{SU}(3) \times \mathrm{SU}(3)^{\prime}$ gauge symmetry.

The quiver diagrams for two examples are depicted in figure 15 . The abelian fugacities for the $5 \mathrm{~d}$ hypermultiplets in the first (or bottom) chamber are given in (3.36). Other abelian charges for the $4 \mathrm{~d}$ fields are fixed by the gauge-global anomaly cancellation and the cubic superpotential couplings. We find

$$
b_{1}=\left(\beta_{4} / \beta_{2}\right)^{1 / 2}, \quad b_{2}=\left(\beta_{4} / \beta_{3}\right)^{1 / 3}, \quad b_{3}=\left(\beta_{5} / \beta_{3}\right)^{1 / 2}, \quad b_{4}=\left(1 / \beta_{3} \beta_{5}\right)^{1 / 2},
$$


for the first quiver diagram and

$$
c_{1}=\left(\beta_{3} / \beta_{2}\right)^{1 / 2}, \quad c_{2}=\left(\beta_{4} / \beta_{2}\right)^{1 / 3}, \quad c_{3}=\left(\beta_{5} / \beta_{2}\right)^{1 / 2}, \quad c_{4}=\left(1 / \beta_{2} \beta_{5}\right)^{1 / 2},
$$

for the second diagram. The first and the second domain walls correspond to the fluxes $F_{1}=\left(0, \frac{1}{8}, \frac{1}{3},-\frac{5}{24}, 0,0\right)_{\beta}$ and $F_{2}=\left(0, \frac{11}{12},-\frac{8}{24},-\frac{1}{12}, 0,0\right)_{\beta}$ respectively. Other basic domain walls can be similarly constructed and generic flux domain walls can be obtained from various combinations of these basic domain walls.

We briefly comment on the other possible choices of the boundary conditions for the $\mathrm{SU}(2)_{i=1,2,3}$ fundamental hypermultiplets. As mentioned, the above basic domain walls choose the same (or the opposite) boundary conditions for each pair of two $\mathrm{SU}(2)_{i}$ fundamental hypers when $\mathcal{T}=\beta$ (or $\mathcal{T}=\gamma$ ). However, we can for example consider a domain wall with opposite boundary conditions for two $\mathrm{SU}(2)_{1}$ fundamental hypers while keeping other boundary conditions and $4 \mathrm{~d}$ chiral fields the same as those drawn in the first quiver in figure 15. In this case, we have a new domain wall with $\beta_{2} / \beta_{1}$ and $\gamma_{2} / \gamma_{1}$ exchanged. Similarly, other choices of boundary conditions for the $\mathrm{SU}(2)_{i=2,3}$ fundamentals can lead to other types of domain walls which we can obtain by exchanging some $\beta_{i}$ and $\gamma_{i}$ 's.

$\boldsymbol{E}_{\mathbf{7}}$ and $\boldsymbol{E}_{\mathbf{8}}$. Lastly, let us discuss the flux domain walls in the affine $E_{7}$ and $E_{8}$ quiver gauge theories. The basic domain walls in these theories can be built by a single interface supporting two copies of affine $E_{7}$ or affine $E_{8}$ quiver gauge symmetries coupled to $5 \mathrm{~d}$ boundary conditions, e.g. $\mathcal{B}=\left\{s_{1}, \cdots, s_{7}\right\}$ for $E_{7}$ or $\mathcal{B}=\left\{s_{1}, \cdots, s_{8}\right\}$ for $E_{8}$, and 4 d chiral fields $q_{i}, \tilde{q}_{i}$ through the cubic superpotentials of the form (3.3). We denote these basic domain walls by $\mathcal{D}=\mathcal{B}_{\mathcal{T}}$. There exists a unique domain wall system for each $\mathcal{D}$. Under the Conjecture stated above, we expect that the flux domain wall systems constructed by gluing the basic domain walls can realize the compactification of the $6 \mathrm{~d} E_{7}$ and $E_{8}$ conformal matter theories with flux on a torus or a tube.

Two basic domain walls in the affine $E_{7}$ quiver theory are given in figure 16. From (3.8), the fugacities $a_{i}$ for the $5 \mathrm{~d}$ hypermultiplets are given by

$$
\begin{array}{lll}
a_{1}=\left(\frac{\beta_{2} \gamma_{1}}{\beta_{1} \gamma_{2}}\right)^{1 / 2}, \quad a_{2}=\left(\frac{\beta_{3}^{2} \gamma_{1} \gamma_{2}}{\beta_{1} \beta_{2} \gamma_{3}^{2}}\right)^{1 / 6}, & a_{3}=\left(\frac{\beta_{4}^{3} \gamma_{1} \gamma_{2} \gamma_{3}}{\beta_{1} \beta_{2} \beta_{3} \gamma_{4}^{3}}\right)^{1 / 2}, \quad a_{4}=\left(\frac{\beta_{1} \beta_{2} \beta_{3} \beta_{4} \beta_{6}^{2} \gamma_{5}^{2}}{\beta_{5}^{2} \gamma_{1} \gamma_{2} \gamma_{3} \gamma_{4} \gamma_{6}^{2}}\right)^{\frac{1}{12}}, \\
a_{5}=\left(\frac{\beta_{1} \beta_{2} \beta_{3} \beta_{4} \beta_{5} \gamma_{6}}{\beta_{6} \gamma_{1} \gamma_{2} \gamma_{3} \gamma_{4} \gamma_{5}}\right)^{1 / 6}, & a_{6}=\left(\frac{\gamma_{7}}{\beta_{7}}\right)^{1 / 2}, & a_{7}=\left(\frac{\gamma_{5} \gamma_{6}}{\beta_{5} \beta_{6}}\right)^{1 / 4},
\end{array}
$$

with the fugacities $\beta_{i}, \gamma_{j}$ for $E_{7} \times E_{7}$ symmetry in the orthogonal basis where the fundamentals of $\mathrm{SU}(2) \times \mathrm{SO}(12) \subset E_{7}$ carry unit charges of $\mathrm{U}(1)_{\beta_{i}}$ or $\mathrm{U}(1)_{\gamma_{i}}$ symmetries. Here $\beta_{7}$ and $\gamma_{7}$ are for the $\mathrm{SU}(2)$ and the rest for the $\mathrm{SO}(12)$. The interface introduces no other anomaly-free abelian symmetry. The abelian charges for the $4 \mathrm{~d}$ fields are uniquely fixed by the gauge-global mixed anomaly cancellation. We find

$$
\begin{array}{ll}
b_{1}=\frac{\beta_{5}}{\beta_{1}}, \quad b_{2}=\left(\frac{\beta_{5}}{\beta_{2}}\right)^{1 / 2}, \quad b_{3}=\left(\frac{\beta_{5}}{\beta_{3}}\right)^{1 / 3}, \quad b_{4}=\left(\frac{\beta_{5}}{\beta_{4}}\right)^{1 / 4}, \quad b_{5}=\left(\frac{\beta_{6}}{\beta_{4}}\right)^{1 / 3}, \\
b_{6}=\left(\frac{\beta_{1} \beta_{2} \beta_{3} \beta_{5} \beta_{6} \beta_{7}}{\beta_{4}}\right)^{1 / 4}, \quad b_{7}=\left(\frac{\beta_{1} \beta_{2} \beta_{3} \beta_{5} \beta_{6}}{\beta_{4} \beta_{7}}\right)^{1 / 2}, & b_{8}=\left(\frac{1}{\beta_{4} \beta_{6}}\right)^{1 / 2},
\end{array}
$$



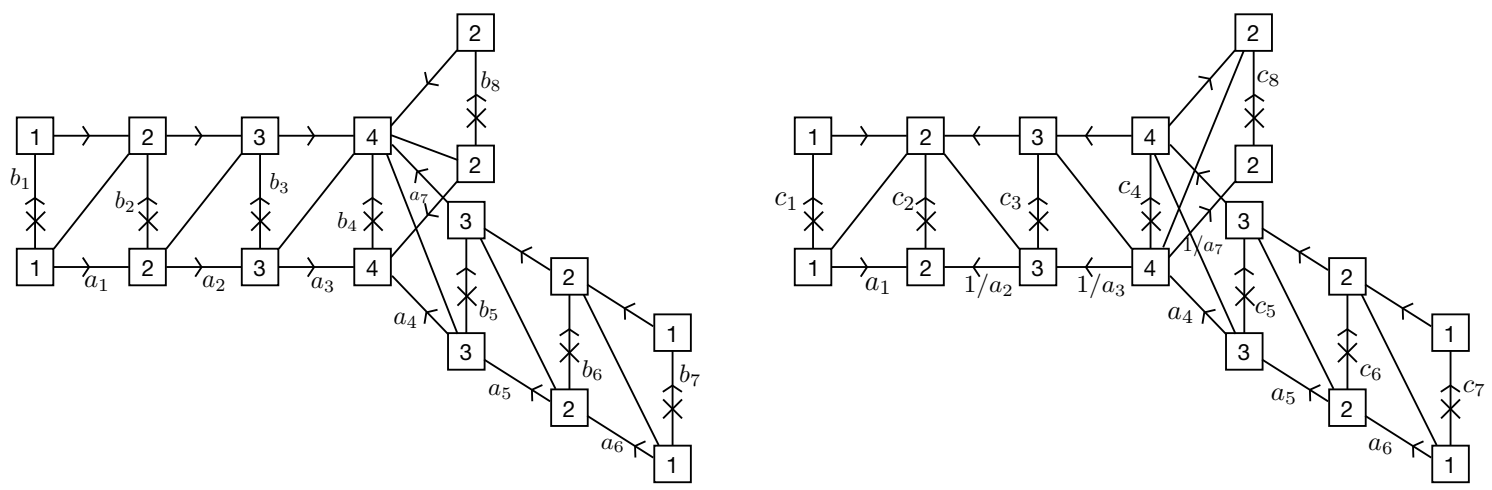

Figure 16. Basic domain walls in the affine $E_{7}$ quiver theory with $\mathcal{D}_{1}=\left\{+{ }^{7}\right\}_{\beta}$ and $\mathcal{D}_{2}=$ $\{+,-,-,+,+,+,-\}_{\beta}$, respectively. The integer numbers $l_{i}$ in the boxes denote the $\operatorname{SU}\left(l_{i} N\right)$ gauge symmetries at the interface.

for the first domain wall with $\mathcal{D}_{1}=\left\{+^{7}\right\}_{\beta}$ and

$$
\begin{array}{ll}
c_{1}=\frac{\beta_{3}}{\beta_{1}}, \quad c_{2}=\left(\frac{\beta_{3}}{\beta_{2}}\right)^{1 / 2}, & c_{3}=\left(\frac{\beta_{4}}{\beta_{2}}\right)^{1 / 3}, \quad c_{4}=\left(\frac{1}{\beta_{2} \beta_{6}}\right)^{1 / 4}, \quad c_{5}=\left(\frac{1}{\beta_{2} \beta_{5}}\right)^{1 / 3}, \\
c_{6}=\left(\frac{\beta_{1} \beta_{3} \beta_{4} \beta_{7}}{\beta_{2} \beta_{5} \beta_{6}}\right)^{1 / 4}, & c_{7}=\left(\frac{\beta_{1} \beta_{3} \beta_{4}}{\beta_{2} \beta_{5} \beta_{6} \beta_{7}}\right)^{1 / 2}, \quad c_{8}=\left(\frac{1}{\beta_{5} \beta_{6}}\right)^{1 / 2},
\end{array}
$$

for the second domain wall with $\mathcal{D}_{2}=\{+,-,-,+,+,+,-\}_{\beta}$. The cubic gauge anomalies are also absent and therefore these domain walls can consistently couple to the 5 d affine $E_{7}$ quiver gauge theory. Using $(3.14)$ with $h^{\vee}=18$, the fluxes are $F_{1}=\left(0,0,0, \frac{2}{9},-\frac{5}{18},-\frac{1}{18}, 0\right)_{\beta}$ for the first domain wall and $F_{2}=\left(0, \frac{5}{18},-\frac{1}{6},-\frac{1}{9}, \frac{1}{6}, \frac{1}{6}, 0\right)_{\beta}$ for the second domain wall. Gluing 12 copies of the first basic domain wall with $\mathcal{D}_{1}=\left\{{ }^{7}\right\}_{\beta}$ leads to a flux domain wall with $F=\left(-\frac{1}{2}, \frac{1}{2}, \frac{3}{2}, \frac{5}{2},-\frac{3}{2},-\frac{1}{2},-1\right)_{\beta}$ corresponding to the 6 d theory on a circle with a unit flux breaking $E_{7} \rightarrow \mathrm{SU}(4) \times \mathrm{SU}(3) \times \mathrm{SU}(2) \times \mathrm{U}(1)$. The circle reduction of this 5 d theory with the flux domain wall yields a $4 \mathrm{~d}$ quiver gauge theory at low energy and the resulting $4 \mathrm{~d}$ theory has the central charges

$$
a=\frac{72 N(3 N-1)^{3 / 2}}{\sqrt{N(4 N-1)}}, \quad c=\frac{9 N(24 N-7) \sqrt{3 N-1}}{\sqrt{N(4 N-1)}},
$$

which precisely coincide with the expected central charges of the $E_{7}$ conformal matter theory on a torus with flux $F$. We also checked that other 't Hooft anomalies of this $4 \mathrm{~d}$ theory match the anomalies obtained by integrating the $6 \mathrm{~d}$ anomaly polynomial in the presence of the flux $F$. One can similarly construct other basic domain walls by choosing different $\mathcal{B}$ and $\mathcal{T}$ and generic flux domain walls from other combinations of the basic domain walls.

In figure 17, we have a basic domain wall for $\mathcal{D}=\left\{+^{8}\right\}_{\beta}$ in the affine $E_{8}$ quiver gauge theory. One can readily check that all cubic gauge anomalies are absent when it is inserted 


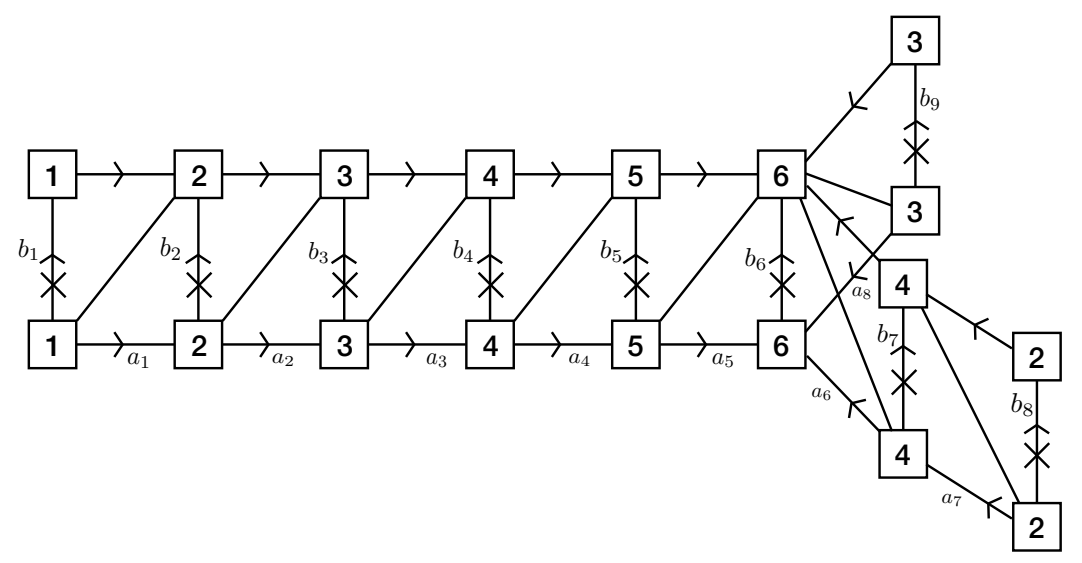

Figure 17. Basic domain wall in the affine $E_{8}$ quiver theory with $\mathcal{D}=\left\{+^{8}\right\}_{\beta}$. The integer numbers $l_{i}$ in the boxes denote the $\mathrm{SU}\left(l_{i} N\right)$ gauge symmetries at the interface.

between two $5 \mathrm{~d}$ affine $E_{8}$ quiver theories. The $5 \mathrm{~d}$ hypermultiplet fugacities $a_{i}$ are

$$
\begin{aligned}
& a_{1}=\left(\frac{\beta_{1} \gamma_{8}}{\beta_{8} \gamma_{1}}\right)^{1 / 2}, \quad a_{2}=\left(\frac{\beta_{2}^{2} \gamma_{1} \gamma_{8}}{\beta_{1} \beta_{8} \gamma_{2}^{2}}\right)^{1 / 6}, \quad a_{3}=\left(\frac{\beta_{3}^{3} \gamma_{1} \gamma_{2} \gamma_{8}}{\beta_{1} \beta_{2} \beta_{8} \gamma_{3}^{3}}\right)^{1 / 12} \\
& a_{4}=\left(\frac{\beta_{4}^{4} \gamma_{1} \gamma_{2} \gamma_{3} \gamma_{8}}{\beta_{1} \beta_{2} \beta_{3} \beta_{8} \gamma_{4}^{4}}\right)^{1 / 20}, \quad a_{5}=\left(\frac{\beta_{5}^{5} \gamma_{1} \gamma_{2} \gamma_{3} \gamma_{4} \gamma_{8}}{\beta_{1} \beta_{2} \beta_{3} \beta_{4} \beta_{8} \gamma_{5}^{5}}\right)^{1 / 30}, \quad a_{6}=\left(\frac{\beta_{1} \beta_{2} \beta_{3} \beta_{4} \beta_{5} \beta_{7}^{3} \beta_{8} \gamma_{6}^{3}}{\beta_{6}^{3} \gamma_{1} \gamma_{2} \gamma_{3} \gamma_{4} \gamma_{5} \gamma_{7}^{3} \gamma_{8}}\right)^{1 / 24} \\
& a_{7}=\left(\frac{\gamma_{7} \prod_{i \neq 7} \beta_{i}}{\beta_{7} \prod_{i \neq 7} \gamma_{i}}\right)^{1 / 8}, \quad a_{8}=\left(\frac{\gamma_{6} \gamma_{7}}{\beta_{6} \beta_{7}}\right)^{1 / 6},
\end{aligned}
$$

in the orthogonal bases where the fundamentals of $\mathrm{SO}(16) \subset E_{8}$ carry charge \pm 1 under $\mathrm{U}(1)_{\beta_{i}}$ or $\mathrm{U}(1)_{\gamma_{i}}$. By demanding the gauge-global mixed anomaly cancellation and the superpotential constraints, we fix the $\mathrm{U}(1)$ charges of the $4 \mathrm{~d}$ chiral fields as

$$
\begin{aligned}
& b_{1}=\frac{\beta_{6}}{\beta_{8}}, \quad b_{2}=\left(\frac{\beta_{6}}{\beta_{1}}\right)^{1 / 2}, \quad b_{3}=\left(\frac{\beta_{6}}{\beta_{2}}\right)^{1 / 3}, \quad b_{4}=\left(\frac{\beta_{6}}{\beta_{3}}\right)^{1 / 4}, \quad b_{5}=\left(\frac{\beta_{6}}{\beta_{4}}\right)^{1 / 5}, \\
& b_{6}=\left(\frac{\beta_{6}}{\beta_{5}}\right)^{1 / 6}, \quad b_{7}=\left(\frac{\beta_{7}}{\beta_{5}}\right)^{1 / 4}, \quad b_{8}=\left(\frac{\prod_{i \neq 5} \beta_{i}}{\beta_{5}}\right)^{1 / 4}, \quad b_{9}=\left(\frac{1}{\beta_{5} \beta_{7}}\right)^{1 / 3} \text {. }
\end{aligned}
$$

From (3.14) with $h^{\vee}=30$, one reads the flux $F=\left(\frac{1}{60}, \frac{1}{60}, \frac{1}{60}, \frac{1}{60}, \frac{7}{60},-\frac{13}{60},-\frac{1}{60}, \frac{1}{60}\right)_{\beta}$ for this basic domain wall. The other basic domain walls can be similarly constructed.

We suggest that the $5 \mathrm{~d}$ affine $E_{8}$ quiver theory with 30 copies of the basic domain wall in figure 17 realizes the $6 \mathrm{~d}\left(E_{8}, E_{8}\right)$ conformal matter theory with flux $F=\left(-\frac{1}{2}, \frac{1}{2}, \frac{3}{2}, \frac{5}{2}, \frac{7}{2},-\frac{5}{2}\right.$, $\left.-\frac{1}{2},-\frac{3}{2}\right)_{\beta}$ on a circle. The flux $F$ is the minimal flux breaking one $E_{8}$ global symmetry to $\mathrm{SU}(5) \times \mathrm{SU}(3) \times \mathrm{U}(1)$. A circle reduction of this $5 \mathrm{~d}$ domain wall configuration leaves a $4 \mathrm{~d}$ quiver gauge theory corresponding to the $6 \mathrm{~d}$ theory with flux $F$ on a torus. Indeed, the central charges of this $4 \mathrm{~d}$ theory

$$
a=\frac{50 \sqrt{3} N(9 N-2)^{3 / 2}}{\sqrt{N(6 N-1)}}, \quad c=\frac{25 N(36 N-7) \sqrt{N(27 N-6)}}{2 \sqrt{N(6 N-1)}}
$$




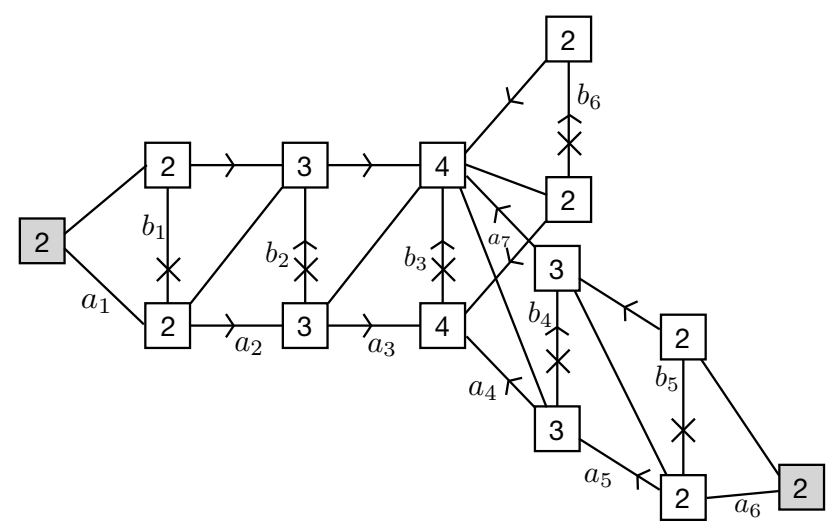

Figure 18. Basic domain walls in the minimal $E_{7}$ theory with $\mathcal{D}_{1}=\left\{+^{7}\right\}_{\beta}$.

agree with those computed by integrating the 6 d anomaly polynomial with $F$. Also, all other anomalies in this $4 \mathrm{~d}$ theory coincide with the anomalies of the $6 \mathrm{~d}$ theory with $F$ on a torus.

Now consider the domain walls in the minimal affine $E_{7}$ and $E_{8}$ quiver gauge theories. When $N=1$, the $\mathrm{U}(1)$ gauge nodes in the quiver diagram are replaced by two fundamental hypermultiplets charged under the adjacent $\mathrm{SU}(2)$ gauge nodes. Domain walls in these theories have almost the same form of those in the non-minimal cases. The differences are the boundary conditions of the $\mathrm{SU}(2)$ fundamental hypers and $4 \mathrm{~d}$ chiral multiplets coupled to these $5 \mathrm{~d}$ fundamental fields. The other parts are the same.

For $E_{7}$, the basic domain walls are defined by $\mathcal{D}=\mathcal{B}_{\mathcal{T}}$ with $\mathcal{B}=\left\{s_{1}, s_{2}, \cdots, s_{7}\right\}$. We choose two fundamentals for an $\mathrm{SU}(2)$ gauge node to have the same (or the opposite) boundary conditions for $\mathcal{T}=\beta$ (or $\mathcal{T}=\gamma$ ). As the minimal $E_{6}$ cases above, the interface connects these boundary conditions of the $\mathrm{SU}(2)$ fundamentals in two sides by using the cubic superpotentials including the $4 \mathrm{~d}$ bifundamental chirals $q_{i}$ charged under $\mathrm{SU}(2)_{i} \times$ $\mathrm{SU}(2)_{i}^{\prime}$. These also hold for the $E_{8}$ cases below with $\mathcal{B}=\left\{s_{1}, s_{2}, \cdots s_{8}\right\}$.

One example of $E_{7}$ is drawn in figure 18 with fugacities $a_{i}$ in (3.44) and

$$
\begin{array}{lll}
b_{1}=\left(\beta_{5} / \beta_{2}\right)^{1 / 2}, & b_{2}=\left(\beta_{5} / \beta_{3}\right)^{1 / 3}, & b_{3}=\left(\beta_{5} / \beta_{4}\right)^{1 / 4}, \\
b_{4}=\left(\beta_{6} / \beta_{4}\right)^{1 / 3}, & b_{5}=\left(\beta_{1} \beta_{2} \beta_{3} \beta_{5} \beta_{6} \beta_{7} / \beta_{4}\right)^{1 / 4}, & b_{6}=\left(1 / \beta_{4} \beta_{6}\right)^{1 / 2},
\end{array}
$$

which are again determined by the gauge-global anomaly cancellation and the superpotential constraints. This quiver diagram describes a basic domain wall with $\mathcal{D}_{1}=\left\{+^{7}\right\}_{\beta}$ and it has the flux $F=\frac{1}{72}(-3,3,1,15,-17,-3,-3)_{\beta}$ in the orthogonal basis of $E_{7}$.

The $E_{8}$ basic domain wall for $\mathcal{D}_{1}=\left\{+^{8}\right\}_{\beta}$ is drawn in figure 19. Here, the 5 d fugacities $a_{i}$ are written in (3.48) and $4 \mathrm{~d}$ fugacities $b_{i}$ are given by

$$
\begin{aligned}
& b_{1}=\left(\beta_{6} / \beta_{1}\right)^{1 / 2}, \quad b_{2}=\left(\beta_{6} / \beta_{2}\right)^{1 / 3}, \quad b_{3}=\left(\beta_{6} / \beta_{3}\right)^{1 / 4}, \quad b_{4}=\left(\beta_{6} / \beta_{4}\right)^{1 / 5} \\
& b_{5}=\left(\beta_{6} / \beta_{5}\right)^{1 / 6}, \quad b_{6}=\left(\beta_{7} / \beta_{5}\right)^{1 / 4}, \quad b_{7}=\left(\prod_{i \neq 5} \beta_{i} / \beta_{5}\right)^{1 / 4}, \quad b_{8}=\left(1 / \beta_{5} \beta_{7}\right)^{1 / 3} \text {. }
\end{aligned}
$$




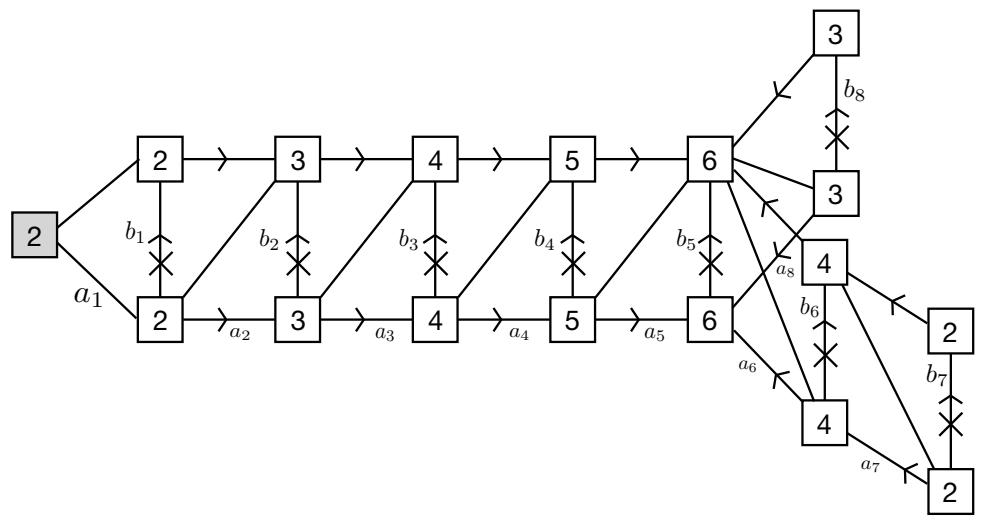

Figure 19. Basic domain walls in the minimal $E_{8}$ theory with $\mathcal{D}_{1}=\left\{+^{8}\right\}_{\beta}$.

This domain wall corresponds to the flux $F=\frac{1}{60}(2,1,1,1,7,-12,-1,-1)_{\beta}$. We will see more examples and tests for our flux domain wall conjectures by reducing them to $4 \mathrm{~d}$ in the next section.

\section{Four dimensions}

We have obtained a conjecture for the theories corresponding to compactifications of ADE conformal matter on a torus with flux for the flavor symmetry. The way we construct the models is by gluing together building blocks which formally correspond to spheres with two maximal punctures and have some flux. In this section we will subject these conjectures to various tests directly in four dimensions. There are two types of checks we can perform, we can compare anomalies and check for enhancements of symmetry. The checks of anomalies we have already discussed so here we will give examples of enhancement of symmetry as well as some dynamical interesting features such as dualities. We will also connect the results to other constructions appearing in the literature.

Note that the two punctured spheres in general have the non abelian flavor symmetry of the models in six dimensions broken down to abelian factors both due to presence of punctures and the flux. The tube theories we have defined also in general, with the exception of minimal $D$ and $E$ conformal matter, have only abelian flavor symmetries which are not associated to the punctures. However, when combining the theories to form a torus and selecting the combination of the two punctured spheres so that the flux is non generic, the symmetry can be enhanced at some loci on the conformal manifold. Such an enhancement is highly non obvious from the four dimensional perspective. One can check such enhancements of symmetry by studying different supersymmetric partition functions, and in particular the supersymmetric index [26-29]. As the models involved have many gauge group factors, the computations, though straightforward, are computationally intense. We will thus restrict in what follows to verifying the claims in some simple examples in which the computations can be performed more easily.

$\boldsymbol{S}$ gluing $\boldsymbol{\Phi}$ gluing and color for punctures. We have derived theories we naturally associate with tubes from our five dimensional discussion. It is natural from the four di- 
mensional point of view to define slightly modified tubes. Note that associated to the punctures we have a collection of chiral fields which are not charged under gauge interactions. In particular we had a bifundamental field between every adjacent factors of the flavor symmetry. It is natural to define the punctures with some of these fields removed. We have a rather arbitrary choice of whether to associate the fields with the puncture or with the gluing procedure. What we gain is that the gluing procedure will become very uniform and also connect directly to previous prescriptions in the literature. The change will be made such that the tori models will be exactly as before and the only difference is with the chiral fields associated to the punctures.

Let us start from a rather abstract discussion of the gluing procedure. Each puncture comes with a set of natural operators which are charged under the symmetry associated to it. We denote these operators by $M_{i}$. These operators generalize the moment maps of class $\mathcal{S}$, which is the $\left(A_{1}, A_{1}\right)$ conformal matter. The choice of the free chiral fields associated to the puncture affects the operators $M_{i}$. There are different types of punctures with the different choices denoted by color, sign, and orientation [7, 8]. As punctures break some of the $G \times G$ symmetry these choices specify what is exactly the preserved symmetry group and what are the anomalies associated to the puncture. Different punctures give rise to different charges under the Cartan of $G \times G$ for $M_{i}$. We glue punctures of same color and sign and opposite orientation by gauging the puncture symmetry and adding a field $\Phi_{i}$ in conjugate representations to $M_{i}$, and coupling them through a superpotential,

$$
W_{\text {gluing }}^{\Phi}=M_{i} \Phi_{i}-\widetilde{M}_{i} \Phi_{i} .
$$

Here $M_{i}$ are the operators of one of the punctures and $\widetilde{M}_{i}$ of the other. We denote this gluing as $\Phi$ gluing $[7,8]$. We will choose the bifundamental fields associated to the puncture in such a way that the fields $\Phi_{i}$ we need to add when gluing two punctures of the same type are most regular. For example, in the A case these will be fields in the fundamental representation of the ith flavor group and antifundamental of $i+1$. See figure 20. This is the choice that was made in $[7,8]$ and we will stick to it here. ${ }^{6}$ All the results in this section are reported using this choice. We also can glue punctures of same color, same orientation, and opposite sign. Punctures of opposite sign have operators $M_{i}$ and $\widetilde{M}_{i}$ in conjugate representations. Thus we turn on the superpotential,

$$
W_{\text {gluing }}^{S}=\widetilde{M}_{i} M_{i}
$$

This gluing will be denoted as $S$ gluing $[7,8,30]$.

For the theories we have defined, to follow the same pattern of gluing as above, we need to add bifundamental fields between symmetry factors on the same side of the duality wall for the tubes only for + boundary conditions and not for minus and call these positive punctures. We can also add the lines for - boundary conditions and not for + and call

\footnotetext{
${ }^{6}$ If we make the choice we have discussed in previous sections then some of the $\Phi_{i}$ fields will be not needed, some will be fundamental-anti-fundamental, and some antifundamental-fundamental. This would make the definition of fields $\Phi_{i}$ rather involved and that is the reason for the choice here.
} 

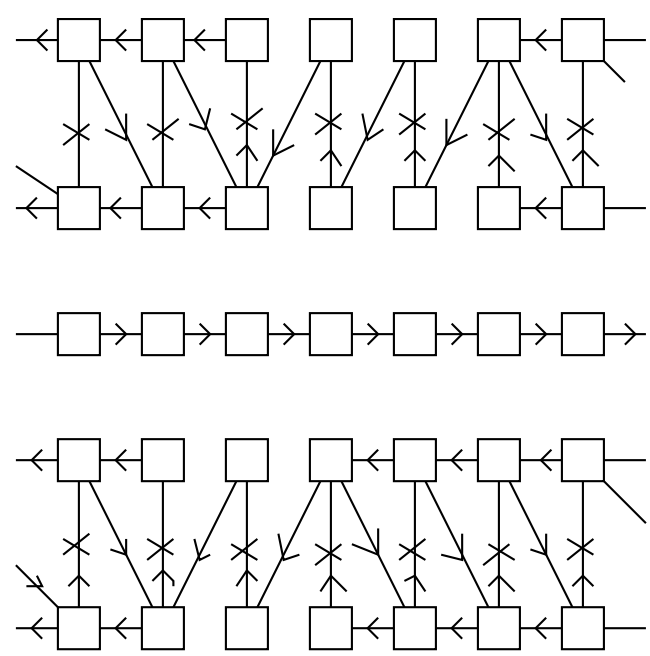

Figure 20. This is $\Phi$ gluing for $A$ theories. The two punctures are of the same sign, opposite orientation, and different colors. The puncture symmetry is $\mathrm{SU}(N)^{k}$. The operators $M_{i}$ are charged under two factors of $\mathrm{SU}(N)$ symmetry. For + boundary conditions they are bifundamental fields and for - they are bi-linear operators. For simplicity we have written arrows on some of the lines and the orientation of the rest is determined by the superpotentials we turn for each face of the quiver. In the middle we have the fields we add when gluing, $\Phi$, in the bifundamental representation of two of the $\mathrm{SU}(N)^{k}$ symmetries.
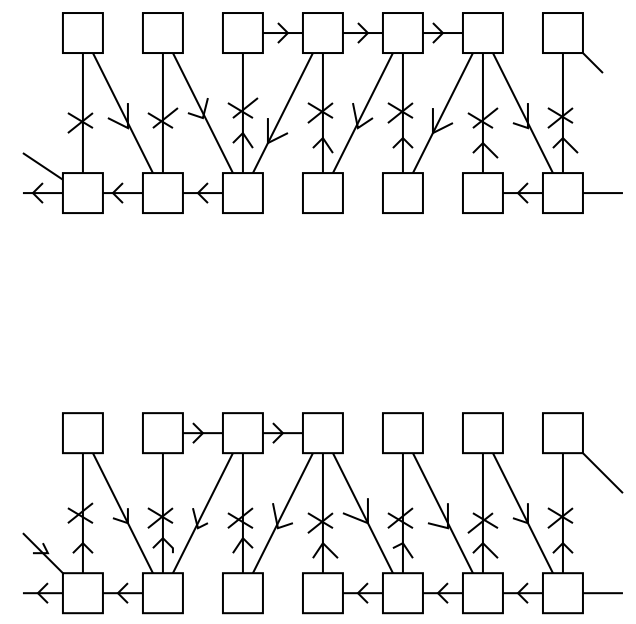

Figure 21. This is $S$ gluing for $A$ theories. The two punctures have opposite sign, same orientation, and different color. We glue the left and right punctures with $\mathrm{S}$ gluing turning on $W_{\text {gluing }}^{S}$ superpotential.

those negative punctures. With this definition of tubes we glue them with $\Phi$ and $S$ gluing. See figures 20 for $\Phi$ gluing and 21 for $S$ gluing. For simplicity we will add fields such that the left puncture is of one sign and the right of opposite sign and build surfaces using $S$ gluing. In this way some of the properties will become simpler. 


\subsection{Examples of $A$}

We start with the case of A-type conformal matter. The case of a single M5 brane, the minimal matter, leads to free models so we will not consider it. The cases of $N>1$ were discussed recently in a variety of papers $[7,8,30,31]$. The compactifications on the torus were considered in [9]. There, the torus with no punctures was constructed by first gluing together theories corresponding to spheres with two maximal and one minimal puncture. Such theories are given by the Wess-Zumino type of models. Then the minimal punctures were closed by turning on vacuum expectation values to certain operators. In particular, it was claimed that in such a manner one can produce models corresponding to flux which is multiple of a $1 / k$. Nevertheless, our discussion has something to add even for this case. The theories we get from this construction generally have flux that is a multiple of $1 / r$ for $r$ an integer obeying $0<r<k$, and thus give theories that are not accessible from the existing construction. ${ }^{7}$ The two construction overlap for theories with integer fluxes for which this provides a different systematic construction of the theories. Let us then discuss some of the general properties of the compactifications and analyze several concrete instances in detail.

The two puncture spheres. The color of a puncture is defined as follows. The symmetry group in the $A$ case is $\mathrm{SU}(k) \times \mathrm{SU}(k) \times \mathrm{U}(1)$. The puncture symmetry is $\prod_{i=1}^{k} \mathrm{SU}(N)_{i}$. We have a cyclic order of the $\mathrm{SU}(N)$ groups coming from the affine Dynkin diagram of type $A$. We have operators $M_{i}$ associated to the puncture for $i=1 \ldots k$. The operators $M_{i}$ are in the bifundamental representation of the $i$-th and $i+1$-th $\mathrm{SU}(N)$ group for positive sign punctures and in the bifundamental of the $i+1$-th and $i$-th $\mathrm{SU}(N)$ group for negative sign punctures. The color is defined by assigning charges to $M_{i}$ under the Cartan of $\mathrm{SU}(k) \times \mathrm{SU}(k)$. We parametrize the Cartan by $\mathrm{U}(1)_{\beta_{l}}$ for one $\mathrm{SU}(k)$ and $\mathrm{U}(1)_{\gamma_{i}}$ for the other. For positive punctures the $M_{i}$ are charged plus one under one of the $\mathrm{U}(1)_{\beta}$ and minus one under one of the $\mathrm{U}(1)_{\gamma}$, and each $M_{i}$ is charged under different symmetries. The choice of the $\mathrm{U}(1)_{\beta}$ and $\mathrm{U}(1)_{\gamma}$ symmetries under which each of the $M_{i}$ operators are charged constitute the color of the puncture. We thus can think of the color, as discussed in previous sections here, as defined by two permutations modulo cyclic transformations, that is the color index takes value in $\left(\sigma_{\beta}, \sigma_{\gamma}\right) \in S_{k} \times S_{k} / \mathbb{Z}_{k}$, where here $S_{k}$ is the symmetric group. For negative punctures the $\mathrm{U}(1)_{\beta}$ charges are negative and $\mathrm{U}(1)_{\gamma}$ are positive. For puncture of color $(1,1)$ the charges of $M_{i}$ are plus one under $\mathrm{U}(1)_{\beta_{i}}$ and minus one under $\mathrm{U}(1)_{\gamma_{i}}$. Punctures of opposite orientation are mirror images under the reflection of the affine Dynkin diagram of $A$. The tubes we have defined have two maximal punctures of different color. We illustrate this in figure 22 .

Because of the superpotential terms, it is clear that the only fields contributing to linear anomalies are the flip fields. Thus defining the flux as in the previous section, we have here $n_{i}=1 / 2$. The flux $Q_{i}$ under $\mathrm{U}(1)_{\beta_{i}}$ is given by the sum of charges of the flip fields under the symmetry divided by $N k$. Because flip fields are not charged under $\mathrm{U}(1)$

\footnotetext{
${ }^{7}$ To be more precise, the theories in question have fractional fluxes and require a central flux element for their consistency, see section 5 in [9] and appendix C in [10]. The theories so far constructed in the literature embed the central flux element for one $\mathrm{SU}(k)$ group in the other $\mathrm{SU}(k)$ group, while the theories considered here embed it in the unbroken part of the same $\mathrm{SU}(k)$ group.
} 


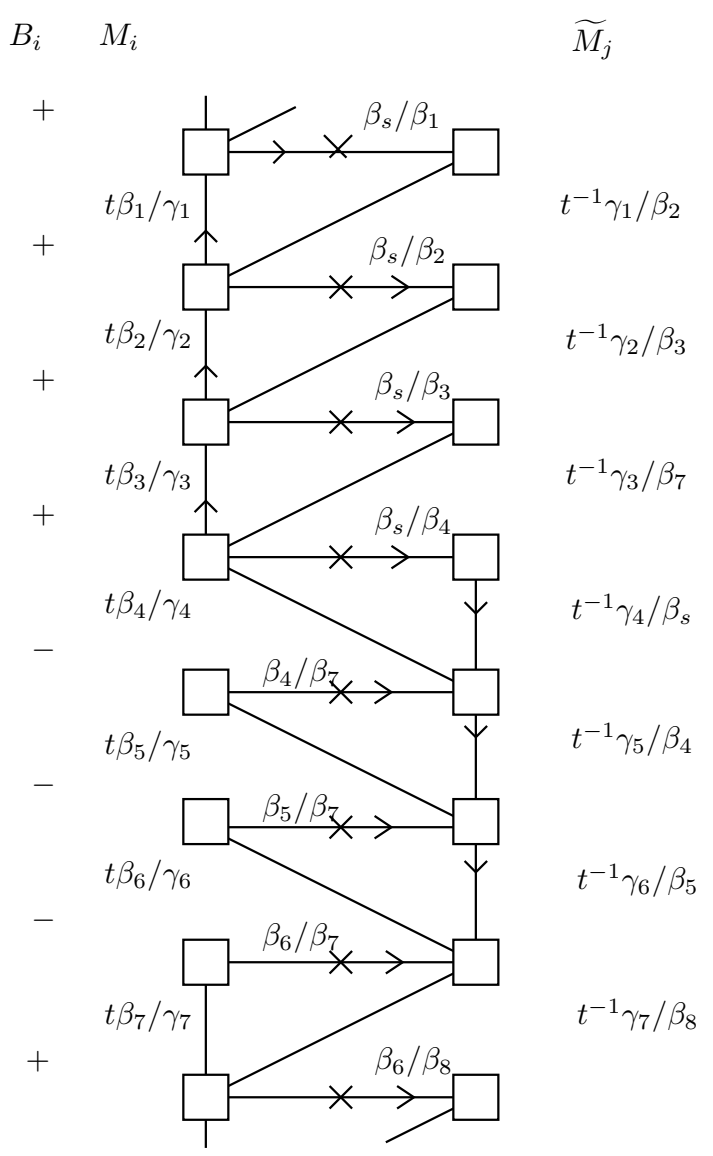

Figure 22. Example of a tube in the four dimensional language. Note this differs from what we have defined before by horizontal fields which are chiral fields associated to punctures. The signs $B_{i}$ correspond to boundary conditions. We denote the charge of operators $M_{i}$ and $\widetilde{M}_{j}$ for the two punctures as manifest in their fugacities. The charges of bifundamental fields are denoted in their fugacities. The missing fugacities can be derived by demanding superpotential terms for every face of the quiver. The $\mathrm{R}$ charges of flipped fields are zero, flip fields are two, and fields which are not flipped are one. The permutation is $(\ldots 12378 \ldots)(\ldots 654 \mathrm{~s} \ldots)$.

symmetries we deduce that the linear anomaly in it is zero. Moreover, because of the supepotentials only the flip fields contribute to $\mathrm{U}(1)_{t \beta_{j}}^{3}$ anomalies. In particular we deduce that the linear anomaly in any $\mathrm{U}(1)$ is the same as cubic up to a factor of $N^{2}$. This agrees with the six dimensional prediction. In general we state as argued in Conjectures that the anomalies agree with the six dimensional prescription if we glue together $l$ tubes if, $\prod_{i=1}^{l} \sigma^{t_{i}} \equiv \sigma=1$. That is if the two colors of maximal punctures are identical. If $\sigma \neq 1$ the anomalies agree for symmetries fixed by $\sigma$. We have verified this statement in numerous cases but did not obtain a rigorous proof.

We will discuss several examples in some detail next.

$\boldsymbol{k}=\mathbf{2}$. Let us consider the case of $k=2$. Here the only choice of $\sigma^{t}$ is the identity as 2 splitting to two non vanishing numbers is $1+1$. This case is identical to the one we obtain by the closing of minimal punctures procedure. The basic tube appears in figure 23 . 


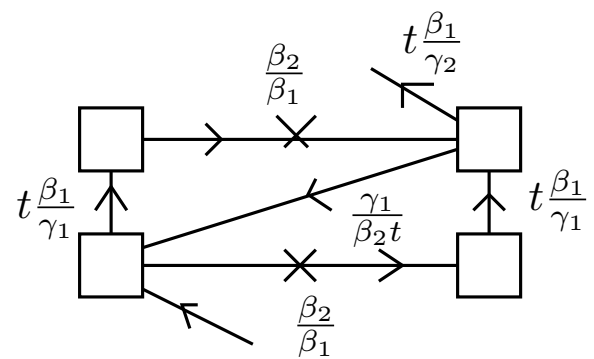

Figure 23. The tube for $k=2$ with flux $(1,-1)$ for the $\beta$ symmetry. This can be obtained through closing punctures of free trinions and through the five dimensional computation. The two punctures are of the same color and sign.

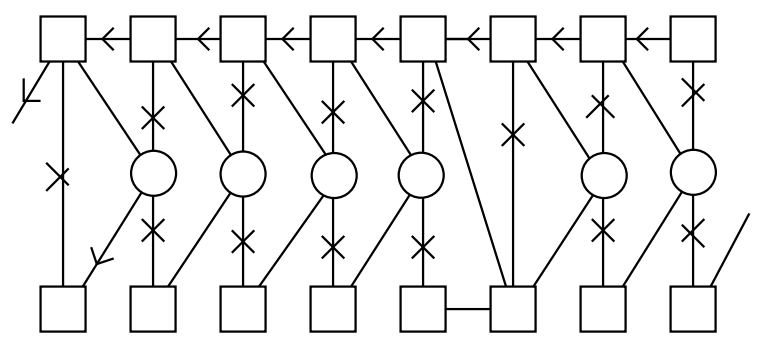

Figure 24. Tube with flux $(0,0, \ldots,-1,0, \ldots, 0,1, \ldots)$ obtained from closing minimal punctures. The two maximal punctures are of the same color and sign.

Let us compute the flux of the model. The $\beta_{1}$ charges of the flip fields are $2 N$ and those of the $\beta_{2}$ fields are $-2 N$ dividing by $k N$ we obtain that the flux is 1 in $\beta_{1}$ and -1 in $\beta_{2}$. This is exactly the flux associated to this tube in [9], and it was checked that all anomalies agree with the six dimensional prescription. Moreover, it was verified in examples that the symmetry observed in the supersymmetric partition functions agrees with the expected symmetry implied by the value of the flux.

Let us here quote a generalization of this tube following the procedure of closing punctures. The tube with two maximal punctures of the same color and same sign and with flux 1 for one of the $\beta_{i}$ and -1 for another while zero for the rest is depicted in figure 24. With this tube any integer flux model can be constructed. Our construction will go beyond this by constructing models with fractional fluxes.

The tube with $\boldsymbol{\sigma}^{t}=(\mathbf{2} 3 \ldots \boldsymbol{k})$. Let us discuss the example of tube with $\sigma^{t}=(23 \ldots k)$ for general $k$. This model fixes one of the $\beta$ symmetries and without generality we can choose it to be $\beta_{1}$. The flux of this model computed from flip fields is $\left(1,-\frac{1}{k},-\frac{1}{k}, \ldots\right.$, $\left.-\frac{1}{k},-\frac{2}{k}\right)$. As the permutation fixes $\beta_{1}$ and all of $\gamma_{i}$, the anomalies involving these symmetries, the R symmetry and the $\mathrm{U}(1)$ agree with six dimensional computation. We can glue several such tubes together to form a torus in such a way that $\beta_{1}$ is always fixed. The flux is fractional for general number of tubes, however for $l$ a multiple of $k-1$ it is a multiple of $(k-1,-1,-1, \ldots,-1)$, and in this case all the symmetries are preserved for the torus and anomalies agree with the computation in six dimensions. It is also easy to see that the quiver in this case is equivalent to a triangulation of the torus with $l$ triangles wrapping 

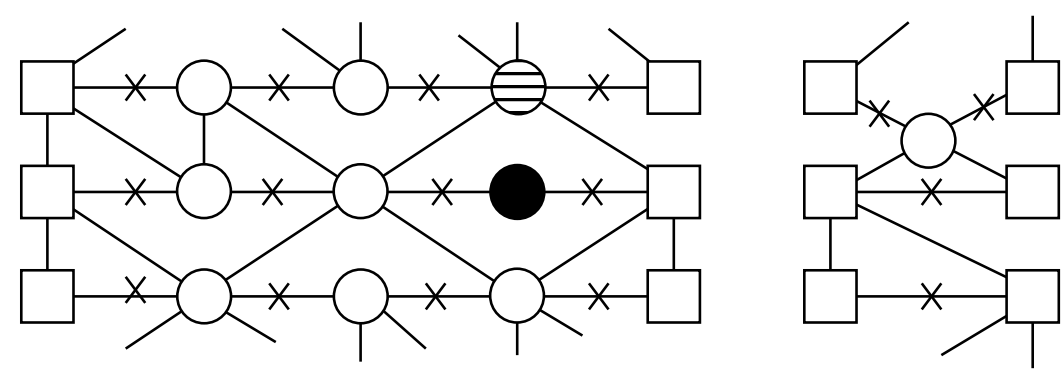

Figure 25. On the left we have a combination of four tubes as in (4.4). Note that one of the nodes, denoted in black, has $N_{f}=N$ and therefore its dynamics leads to it being Higgsed and the groups connected to it identified. Following this, the node denoted with dashed lines becomes $2 N=N_{f}$. After performing Seiberg duality on this node, as well as a sequence of similar manipulations, we obtain the quiver on the right hand side. This is the same as the one obtained by closing punctures in [9] as in figure 24, if one flips the sign of one of the punctures as our tubes have punctures of different signs.

one cycle and $k$ another, with one side of each triangle flipped. The flipped sides form $k$ lines wrapping the cycle with $l$ triangles. This is also the quiver that one would obtain if one glues together tubes that one naively associates to all same sign boundary conditions.

$\boldsymbol{k}=\mathbf{3}$ and $\boldsymbol{k}=\mathbf{4}$. Let us here also discuss the two less obvious cases in some detail. Let us first take $k=3$. We have one type of tube as we can split $3=2+1$, which is the tube discussed in the previous subsection. We can define a similar tube, but with the permutation and flux in the $\gamma$ symmetries. We then have the freedom of gluing them together in a variety of ways. For example, we can take the following tubes,

$$
\sigma^{t_{a}}=(23), \quad \sigma^{t_{b}}=(13), \quad \sigma^{t_{c}}=(23), \quad \sigma^{t_{e}}=(12) .
$$

We have that $\sigma^{t_{a}} \sigma^{t_{b}} \sigma^{t_{c}} \sigma^{t_{e}}=1$ and thus all anomalies are expected to agree with six dimensions. Moreover, the flux is,

$$
\left(1,-\frac{1}{3},-\frac{2}{3}\right)+\left(-\frac{1}{3}, 1,-\frac{2}{3}\right)+\left(1,-\frac{1}{3},-\frac{2}{3}\right)+\left(-\frac{2}{3},-\frac{1}{3}, 1\right)=(1,0,-1)
$$

This is a flux one can obtain from closing punctures as in [9], and as the two have same anomalies and expected symmetry they should be dual to each other. This should be possible to show using Seiberg duality [32], see figure 25.

For $k=4$ we already have a richer variety of constructions. In addition to the (123) tube we also have tubes associated with the (12)(34) and (13)(24) permutations. The former has flux $\left(\frac{3}{4}, \frac{1}{4},-\frac{1}{4},-\frac{3}{4}\right)$ and the latter tube flux $\left(\frac{1}{2},-\frac{1}{2}, \frac{1}{2},-\frac{1}{2}\right)$. We can read off these fluxes easily from the flip fields. If we glue the first tube to itself we obtain the flux $(1,1,-1,-1)$, while doing the same for the second gives the flux $(1,-1,1,-1)$. We thus can construct these tubes from the ones we obtained by closing punctures and verify that the two constructions agree upon making use of dualities. 


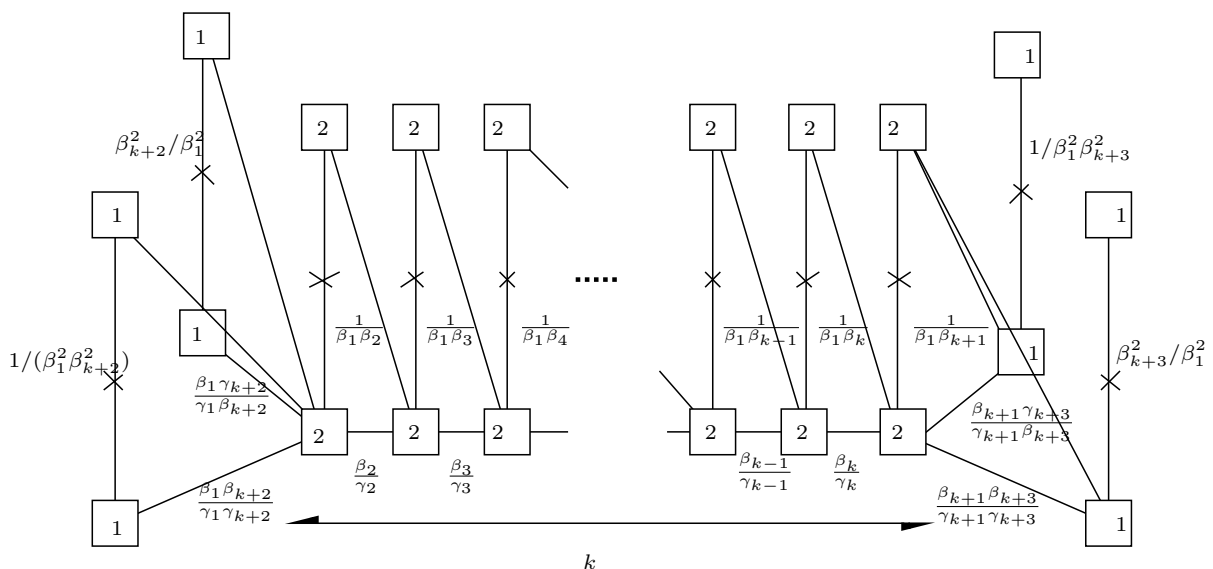

Figure 26. The tube with all plus boundary conditions. We only show the charges for horizontal lines, which are the $M_{i}$, and for the flipped fields. The other charges are determined by the triangular superpotentials. Here flavor groups denoted by 2 are $\mathrm{SU}(2 N)$ and by 1 are $\mathrm{SU}(N)$. Note that for $N=1$ the flip and flipped fields on the edges form mass terms and decouple. The two punctures are of opposite sign and we glue them with $S$ gluing. We can see that the linear anomaly of this theory comes only from flip fields.

\subsection{Examples of $D$}

The discussion here will follow the general ideas of the previous section. In particular we start by defining the color of the punctures. The punctures have $\mathrm{SU}(2 N)^{k} \times \mathrm{SU}(N)^{4}$ symmetry for $D_{k+3}$ case. We have $k+3$ operators associated to the puncture and we will denote them as $M_{1,2}^{a}, M_{1,2}^{b}$ and $M_{i}$ with $i \in\{1, \ldots, k-1\}$. The punctures have a color which is defined by a label expected to take value in $W_{D_{k+3}} \times W_{D_{k+3}}$, which is the product of the two Weyl groups of $D_{k+3}$, possibly moded by some discrete symmetry. The tubes then can be viewed as associated to an element of the Weyl group of one of the two $D_{k+3}$ groups.

We parametrize one of the $D_{k+3}$ by $\beta_{i}$ and another by $\gamma_{i}$. We choose the fugacities so that the vector representation character is,

$$
\mathbf{2 k}+\mathbf{6}_{\gamma}=\sum_{i=1}^{k+3} \gamma_{i}^{\mp 2 N}, \quad \mathbf{2 k}+\mathbf{6}_{\beta}=\sum_{j=1}^{k+3} \beta_{j}^{\mp 2 N} .
$$

The basic tube of figure 26 acts on color by permuting clockwise $\beta_{1} \ldots \beta_{k+1}$ and by taking $\beta_{k+2}$ to $1 / \beta_{k+2}$ and $\beta_{k+3}$ to $1 / \beta_{k+3}$. We can compute the charges of the flip fields to be,

$$
\beta_{1}: \quad 2 N(k+4), \quad \beta_{k+3}, \beta_{k+2}: 0, \quad \beta_{l \neq 1, k+3, k+2}: 2 N .
$$

All other symmetries have zero charge. In particular let us now glue $k+1$ such tubes together to torus. If $k$ is odd then all symmetries are preserved, and if $k$ is even $\beta_{k+3}$ and $\beta_{k+2}$ are broken because of the Weyl $\mathbb{Z}_{2}$ action of the tube. The charges then are,

$$
\beta_{l \neq k+3, k+2}: 2 N(2 k+4), \quad \beta_{k+3}, \beta_{k+2}: 0,
$$


We note that $h^{\vee}$ for $D_{k+3}$ is $2 k+4$ and thus following our usual logic we identify the flux as being proportional to $2 N$. Checking other anomalies we find that the flux associated to the torus is one in $\frac{1}{k+1} \sum_{j=1}^{k+1} \mathrm{U}(1)_{\beta_{j}}$. In particular this means that to compute the flux of a theory we compute the charge of the flip fields and divide by $2 N(2 k+4)$.

We can choose different boundary conditions for the various fields. The tube will implement then the action of various elements of the Weyl symmetry group. This will involve rotation of $\beta_{i}$ and flips. We will discuss this in detail in some cases.

Affine quiver. We can take the tube above and glue the two punctures together. The theory one obtains is the $\mathcal{N}=2 \mathrm{D}$ shaped affine quiver with the adjoint (or more correctly bifundamentals of same group) fields flipped. All $\beta$ symmetries are broken save the diagonal combination of $\beta_{i} \neq k+3, k+2$. This $\mathrm{U}(1)$ corresponds to the symmetry under which the adjoints in $\mathcal{N}=2$ are charged. All $\gamma$ symmetries survive and we expect the symmetry to enhance to $D_{k+3}$. Note that for $k=1$ and $N=2$ this is the symmetry of the Lagrangian. For other $k$ and $N$ the Lagrangian exhibits only the $\mathrm{U}(1)$ symmetries. However, because of $\mathcal{N}=2$ dualities the index will be organized in representations of $D_{k+3}$. Moreover, the models have $\mathcal{N}=1$ conformal manifold on which the symmetry can enhance to $D_{k+3}$. Note that the dimension of the conformal manifold is $2 k+6$ with all the symmetry preserved. This means that the index at order $q p$, which does not depend on any flavor, is $k+2$. If the symmetry enhances to $D_{k+3}$ that means we will have the contribution of the currents for $D_{k+3} \times \mathrm{U}(1)$. It is then conceivable that we have $k+3$ marginal operators which are singlets of the symmetry and another marginals in the adjoint of the non abelian group.

$\boldsymbol{D}_{4}$. Let us discuss the case of $D_{4}$. In figure 27 we depict three different tubes for this case. The tube on the left corresponds to flux $\left(\frac{5}{6}, \frac{1}{6}, 0,0\right)$ in $\beta$, the tube on the right to flux $\left(\frac{1}{2},-\frac{1}{2}, 0,0\right)$, and the tube on the bottom to flux $\left(\frac{2}{3},-\frac{1}{3}, 0,-\frac{1}{3}\right)$. The tubes correspond to the following Weyl symmetry,

$$
\begin{aligned}
\frac{5}{6}, \frac{1}{6}, 0,0: & \beta_{1} \leftrightarrow \beta_{2}, \quad \beta_{3} \rightarrow 1 / \beta_{3},, \beta_{4} \rightarrow 1 / \beta_{4}, \\
\frac{1}{2},-\frac{1}{2}, 0,0: & \beta_{1} \leftrightarrow 1 / \beta_{2}, \beta_{3} \rightarrow 1 / \beta_{3},, \beta_{4} \rightarrow 1 / \beta_{4}, \\
\frac{2}{3},-\frac{1}{3}, 0,-\frac{1}{3}: & \beta_{1} \leftrightarrow 1 / \beta_{4}, \beta_{3} \rightarrow 1 / \beta_{3},, \beta_{2} \rightarrow 1 / \beta_{2} .
\end{aligned}
$$

For general values of $N$ we can easily compute the indices of some models corresponding to closed surfaces. For example let us glue two copies of the same tube together. All tubes of figure 27 will give equivalent theories. We can discuss the left tube which will give flux $(1,1,0,0)$ for $\beta$ and zero for $\gamma$. For general $N$ the gauge invariant operators are the baryons, flip fields, and operators corresponding to closed loops on the quiver. The baryons have large dimensions and flip fields are free fields for general $N$. The operators of smallest charge are then built from flipped fields winding the quiver and from operators corresponding to faces. Additional operators contributing to the index are given by gaugino bilinears for each gauge group and by $\bar{\psi}_{Q} Q$ operators for each field. We have 26 of the latter operators and have ten gaugino bilinears. We also have sixteen faces. The index is 


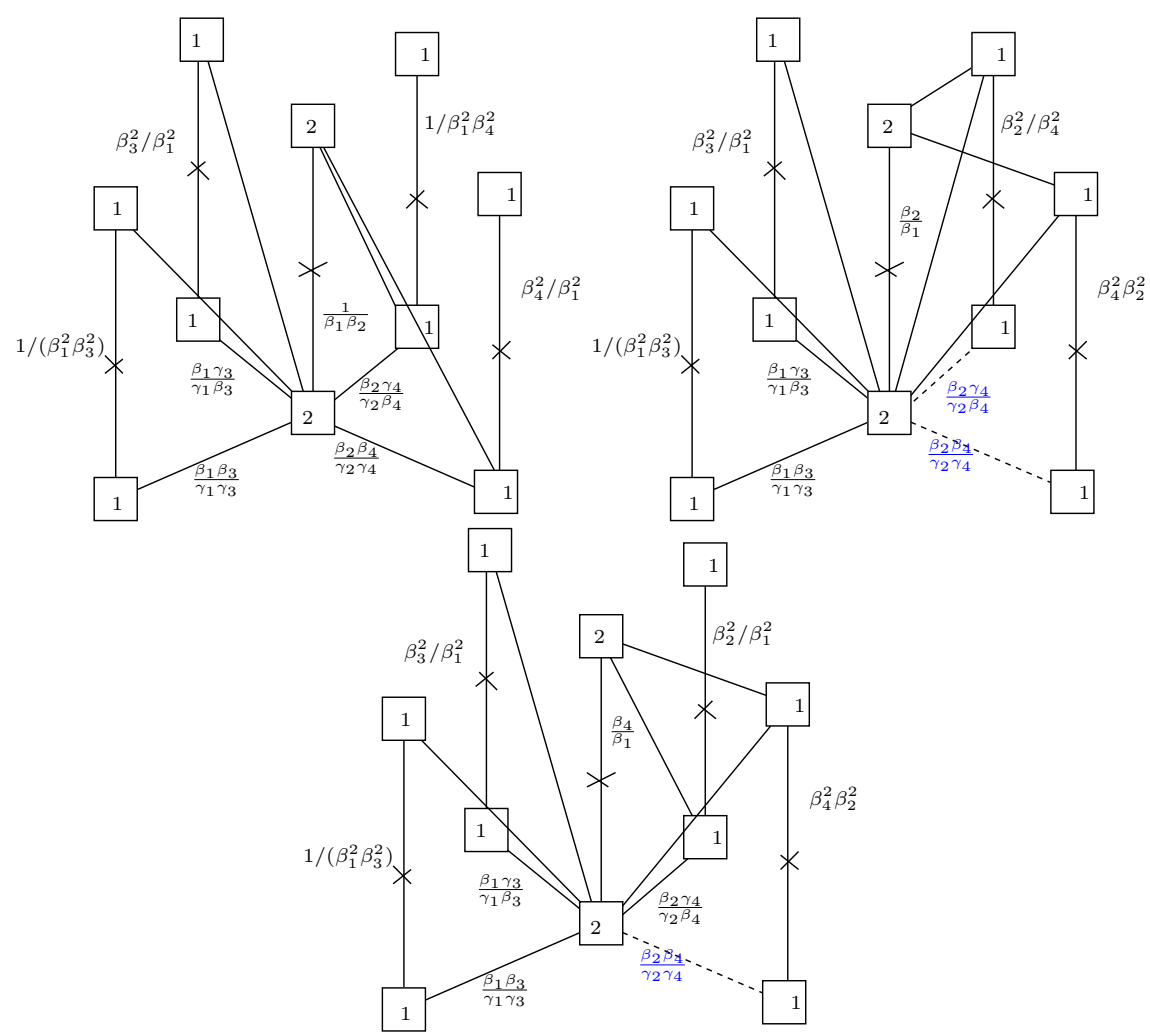

Figure 27. Three different tubes for the $D_{4}$ case. The two punctures are of opposite sign and different colors. The dotted line represents the $M_{i}$ which in the cases the line appears are composites. The groups labeled by 1 are $\mathrm{SU}(N)$ and groups labeled by 2 are $\mathrm{SU}(2 N)$.

then, ignoring flip fields which are free,

$$
1+\frac{5}{\beta_{1}^{2} \beta_{2}^{2}} q^{\frac{2}{3}} p^{\frac{2}{3}}+\cdots
$$

The superconformal $\mathrm{R}$ charge is the free one. The index at order $q p$ is vanishing. The order $q p$ in index computations using the superconformal R-symmetry counts the marginal operators minus the conserved currents for global symmetries [28]. The symmetry $\beta_{1} \beta_{2}$ is the symmetry which has the flux. The $D_{4} \times D_{4}$ symmetry is broken to $\mathrm{SU}(2)^{3} \times \mathrm{U}(1) \times \mathrm{SO}(8)$. At this order of the index we see the $\mathrm{U}(1)$ symmetry. At zero coupling we can count the dimension of the manifold of conformal couplings. The number of symmetries is 26 . The number of marginal operators is 26 . On a general point of the conformal manifold only eight symmetries are not broken. This indicates that the dimension of the conformal manifold is eight. We expect then to have marginal operators in the adjoint of $\mathrm{SO}(8)$ and $\mathrm{SU}(2)^{3} \times \mathrm{U}(1)$. These operators would give eight exactly marginal directions. Thus we conclude that it can be that on some point of the manifold the symmetry enhances.

We can also try to understand what are the states charged under the $\gamma$ symmetry. The generic states charged under these symmetries are baryonic operators built from bifundamental operators of two $\mathrm{SU}(N)$ groups which are composites of two bifundamentals 
of $\mathrm{SU}(2 N) \mathrm{SU}(N)$. The contribution to the index of these is,

$$
q^{\frac{2 N}{3}} p^{\frac{2 N}{3}} \beta_{1}^{N} \beta_{2}^{N}\left[\left(\beta_{3}^{N} \beta_{4}^{N}+\frac{1}{\beta_{3}^{N}} \frac{1}{\beta_{4}^{N}}\right) \mathbf{8}_{s}+\left(\frac{\beta_{3}^{N}}{\beta_{4}^{N}}+\frac{\beta_{4}^{N}}{\beta_{3}^{N}}\right) \mathbf{8}_{c}\right] .
$$

Here,

$$
\begin{aligned}
& \boldsymbol{8}_{s}=\left(\gamma_{1}^{N} \gamma_{3}^{N}\right)^{ \pm 1}\left(\gamma_{2}^{N} \gamma_{4}^{N}\right)^{ \pm 1}+\left(\gamma_{1}^{N} / \gamma_{3}^{N}\right)^{ \pm 1}\left(\gamma_{2}^{N} / \gamma_{4}^{N}\right)^{ \pm 1} \\
& \mathbf{8}_{c}=\left(\gamma_{1}^{N} / \gamma_{3}^{N}\right)^{ \pm 1}\left(\gamma_{2}^{N} \gamma_{4}^{N}\right)^{ \pm 1}+\left(\gamma_{1}^{N} \gamma_{3}^{N}\right)^{ \pm 1}\left(\gamma_{2}^{N} / \gamma_{4}^{N}\right)^{ \pm 1}
\end{aligned}
$$

We see that the operators form representations of $\mathrm{SO}(8) \times \mathrm{SU}(2)^{3} \times \mathrm{U}(1)$. Note that $\beta_{3}^{N} \beta_{4}^{N}+\frac{1}{\beta_{3}^{N} \beta_{4}^{N}}$ and $\beta_{3}^{N} / \beta_{4}^{N}+\beta_{4}^{N} / \beta_{3}^{N}$ are characters of the two spinor representations of $\mathrm{SU}(2) \times \mathrm{SU}(2) \sim \mathrm{SO}(4)$. Note that for $N=1$ there are additional operators at low charges and this is the special case of the E-string which is discussed in detail in [10].

We can also combine different tubes together. Note that because of the non trivial Weyl action on the color, the order of gluing tubes actually can matter. For example combining the two tubes on the left and then two tubes on the right the theory has flux $(2,0,0,0)$. However combining the left tube to the right one and then taking two copies of this gives different flux, $(0,0,0,0)$. This theory is singular. In the first case the symmetry is actually enhancing to $\mathrm{U}(1) \times \mathrm{SO}(6) \times \mathrm{SO}(8)$, which further enhances to $\mathrm{U}(1) \times \mathrm{SO}(14)$ in the case of $N=1$.

$D_{5}$ minimal. In this subsection we consider some examples for the case of minimal $D_{5}$. In figure 28 we have drawn three tubes for this case, where we concentrate only on tubes with no flux in the $\mathrm{SU}(4) \times \mathrm{SU}(4)$ groups rotating the flavors at the ends of the quiver. Using our prescription, we associate with tube I the flux $\left(\frac{1}{2}, \frac{1}{4}, \frac{1}{4}, 0\right)$, with tube II the flux $\left(\frac{1}{4},-\frac{1}{4}, \frac{1}{2}, 0\right)$ and with tube III the flux $\left(\frac{1}{4}, 0, \frac{1}{2},-\frac{1}{4}\right)$. Here the fluxes are oriented as $\left(F_{\beta_{1}}, F_{\beta_{2}}, F_{\beta_{3}}, F_{\gamma_{2}}\right)$, and for brevity we ignore the fluxes in $\beta_{4-5}, \gamma_{1}$ and $\gamma_{3-5}$ as these are zero for these tubes and for theories made of them. We note that to all of these tubes corresponds the same flux up to a Weyl transformation.

We next try to test these conjectures in various ways. As noted previously the anomalies for tubes generally do not match the $6 d$ expectations, and these tubes are no different. However, it is possible that those for closed surfaces will work. To test these we next consider various closed surfaces that can be built from these tubes.

First we note that closing the tubes on to themselves leads to the same quiver for each tube. The quiver in question is an $\mathcal{N}=2 \mathrm{SU}(2) \times \mathrm{SU}(2)$ quiver gauge theory with a bifundamental hypermultiplet and two fundamental hypermultiplet for each of the $\mathrm{SU}(2)$ gauge groups. Additionally there are chiral fields coming from the flipped bifundamental as well as the flipping fields. These theories correspond to the flux $\left(\frac{1}{3}, \frac{1}{3}, \frac{1}{3}, 0\right)$, up to a Weyl transformation. This comes about as when closing the tubes we are forced to identify the $3 \mathrm{U}(1)$ groups with the flux, which forces it to distribute evenly between them leading to this structure.

The gluing breaks part of the global symmetry leaving us with a symmetry of rank 6 . This agrees with what the $6 d$ expectation as for this value of flux to be consistent we must include center fluxes breaking the global symmetry to $\mathrm{U}(1) \times \mathrm{SO}(11)$. We can preform 

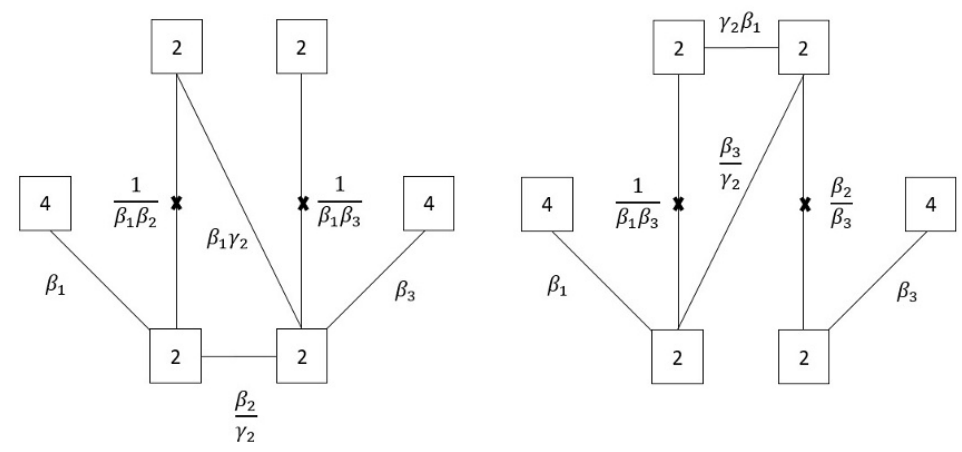

।

II

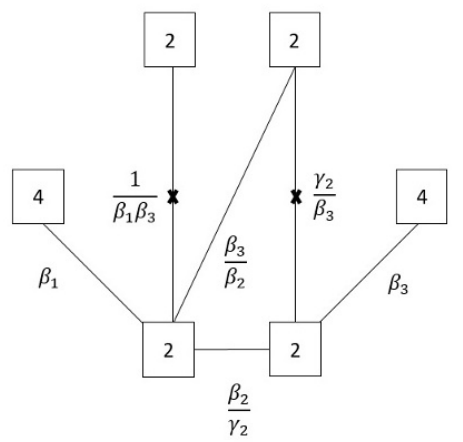

III

Figure 28. A collection of tubes in the minimal $D_{5}$ case with no flux in the $\mathrm{SU}(4) \times \mathrm{SU}(4)$ groups rotating the flavors at the ends of the quiver.

various consistency checks, particularly we can match anomalies which agree with the $6 d$ expectations. We can also argue that the index should form characters of $\mathrm{U}(1) \times \mathrm{SO}(11)$ in the same manner as for the previous affine quivers.

We can also consider connecting each tube to itself to build theories associated with larger values of fluxes. However, in order to connect the tubes we need to cycle symmetries with flux in them, meaning that the fluxes of the resulting tube are not just twice that of the individual tubes. Particularly, when connecting three tubes we get to flux $(1,1,1,0)$, and those related by Weyl transformations for the other tubes. These can be closed to a torus without breaking symmetries with flux, and we can preform similar consistency checks on these theories as well, such as matching anomalies. More intricate checks are given by connecting two different tubes, and we next consider each in turn.

First we consider gluing tubes $I$ and $I I$. Due to the cycling of the global symmetry necessary when connecting the two tubes, we need to shift the fluxes for tube $I I$. Summing the two fluxes, we associate with the resulting tube the flux $\left(\frac{1}{2}, \frac{1}{4}, \frac{1}{4}, 0\right)+\left(\frac{1}{2},-\frac{1}{4}, \frac{1}{4}, 0\right)=$ $\left(1,0, \frac{1}{2}, 0\right)$.

We can next close the tube to a torus. When doing this we need to turn off the symmetries associated with $\beta_{2}$ and $\beta_{3}$. The resulting theory is shown in figure 29 . As we 


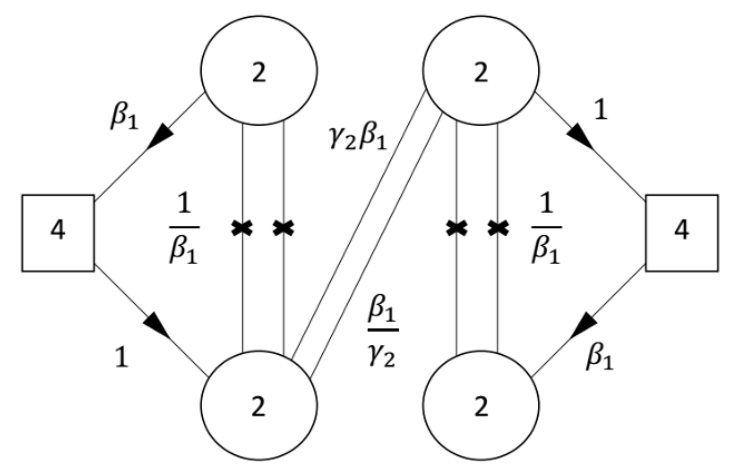

Figure 29. The theory resulting from gluing the tubes $I$ and $I I$ together.

were required to turn $\beta_{3}$ off, we associate with this theory the flux $(1,0,0,0)$. We can test this in various ways. First we can compare anomalies where we find that they indeed match.

As a more intricate test we can consider the superconformal index. The general form of the flux is expected to preserve $\mathrm{U}(1) \times \mathrm{SO}(18)$ global symmetry, yet this specific value requires, for consistency, also a central flux element that breaks some of the global symmetry leading to the breakdown of $\beta_{2}$ and $\beta_{3}$. The resulting symmetry, while dependent on the choice of central element, is known to be at most $\mathrm{U}(1) \times \mathrm{SO}(15)$. We can try to test this by evaluating the superconformal index and see if the appearing operators can be merged so as to form characters of this symmetry.

First we should consider the superconformal R-symmetry. Using a-maximazation we find it to be: $\mathrm{U}(1)_{R}^{s c}=\mathrm{U}(1)_{R}^{6 d}-\sqrt{\frac{11}{45}} \mathrm{U}(1)_{\beta_{1}}$. With this R-symmetry, we find no operators violating the unitarity bound and so no contradiction with this theory flowing to an interacting SCFT. We can then evaluate the index, where, for the purpose of the evaluation, we shall use the R-symmetry $\mathrm{U}(1)_{R}^{6 d}-\frac{1}{2} \mathrm{U}(1)_{\beta_{1}}$, which is quite close to the superconformal one, as $\frac{1}{2}-\sqrt{\frac{11}{45}} \approx 0.0056$. We find:

$$
\begin{aligned}
I= & 1+(p q)^{\frac{1}{2}}\left(\frac{2}{\beta_{1}^{2}}+\beta_{1}^{2}\left(4+\chi[\mathbf{1 5}]_{\mathrm{SO}(15)}\right)\right)+(p q)^{\frac{1}{2}}(p+q) \beta_{1}^{2}\left(3+\chi[\mathbf{1 5}]_{\mathrm{SO}(15)}\right) \\
& +p q\left(\frac{3}{\beta_{1}^{4}}+5+\chi[\mathbf{1 5}]_{\mathrm{SO}(15)}+\beta_{1}^{4}\left(\chi[\mathbf{1 1 9}]_{\mathrm{SO}(15)}+4 \chi[\mathbf{1 5}]_{\mathrm{SO}(15)}+9\right)\right)+\ldots
\end{aligned}
$$

Here we have already written the index in characters of the expected $\mathrm{U}(1) \times \mathrm{SO}(15)$ global symmetry, where: $\chi[\mathbf{1 5}]_{\mathrm{SO}(15)}=1+\gamma_{2}^{2}+\frac{1}{\gamma_{2}^{2}}+\chi[\mathbf{6}, \mathbf{1}]+\chi[\mathbf{1}, \mathbf{6}]$. This shows that the index can indeed be written in characters of $\mathrm{U}(1) \times \mathrm{SO}(15)$, at least to the evaluated order.

We next consider gluing tubes $I I$ and $I I I$. Due to the cycling of the global symmetry necessary when connecting the two tubes, we now need to shift the fluxes for tube III. Again summing the two fluxes, we associate with the resulting tube the flux $\left(\frac{1}{4},-\frac{1}{4}, \frac{1}{2}, 0\right)+$ $\left(\frac{1}{2}, 0, \frac{1}{4},-\frac{1}{4}\right)=\left(\frac{3}{4},-\frac{1}{4}, \frac{3}{4},-\frac{1}{4}\right)$.

We can next close the tube to a torus. When doing this we are forced to identify $\beta_{3}=\frac{1}{\gamma_{2}}$ and $\beta_{1}=\frac{1}{\beta_{2}}$. The resulting theory is shown in figure 30. Due to the required identification, we associate with this theory the flux $\left(\frac{1}{2},-\frac{1}{2}, \frac{1}{2},-\frac{1}{2}\right)$. We next test this in 


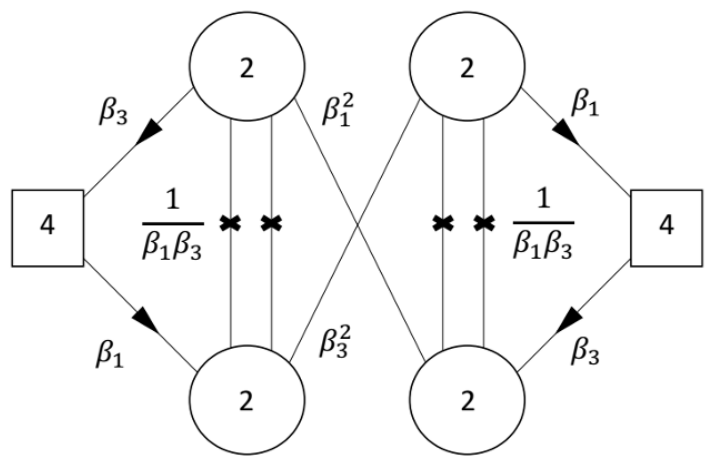

Figure 30. The theory resulting from gluing the tubes $I I$ and $I I I$ together.

various ways. The basic test is to compare anomalies against those expected from $6 d$, where we indeed find that they match.

We can again consider evaluating the superconfomal index looking for character structure of the expected global symmetry. Here the structure of the flux is that of $U(1) \times$ $\mathrm{SU}(4) \times \mathrm{SO}(12)$ preserving flux. However due to the fractional flux, part of the symmetry is broken so that at most $\mathrm{U}(1) \times \mathrm{SU}(2) \times \mathrm{SO}(12)$ can be preserved. This is manifested in the construction by the identification that we were forced to perform upon closing the tube.

We start by studying the superconformal R-symmetry using a-maximazation. We find it to be: $\mathrm{U}(1)_{R}^{s c}=\mathrm{U}(1)_{R}^{6 d}-\sqrt{\frac{11}{126}} \mathrm{U}(1)_{\beta_{1}}-\sqrt{\frac{11}{126}} \mathrm{U}(1)_{\beta_{3}}$. With this R-symmetry, we find no operators violating the unitarity bound and so no contraction with this theory flowing to an interacting SCFT. we can then evaluate the index, where, for the purpose of the evaluation, we shall use the R-symmetry $\mathrm{U}(1)_{R}^{6 d}-\frac{1}{3} \mathrm{U}(1)_{\beta_{1}}-\frac{1}{3} \mathrm{U}(1)_{\beta_{3}}$, which is quite close to the superconformal one, as $\frac{1}{3}-\sqrt{\frac{11}{126}} \approx 0.04$. We find:

$$
\begin{aligned}
I= & 1+(p q)^{\frac{1}{3}} \beta_{1}^{2} \beta_{3}^{2}\left(3+\chi[\mathbf{3}]_{\mathrm{SU}(2)}\right) \\
& +(p q)^{\frac{2}{3}}\left(\frac{2}{\beta_{1}^{2} \beta_{3}^{2}}+\beta_{1}^{4} \beta_{3}^{4}\left(\chi[\mathbf{5}]_{\mathrm{SU}(2)}+3 \chi[\mathbf{3}]_{\mathrm{SU}(2)}+7\right)+\beta_{1} \beta_{3} \chi[\mathbf{2}]_{\mathrm{SU}(2)} \chi[\mathbf{1 2}]_{\mathrm{SO}(12)}\right)+\ldots
\end{aligned}
$$

Here we have already written the index in characters of the expected $\mathrm{U}(1) \times \mathrm{SU}(2) \times$ $\mathrm{SO}(12)$ global symmetry, where: $\chi[\mathbf{2}]_{\mathrm{SU}(2)}=\frac{\beta_{1}}{\beta_{3}}+\frac{\beta_{3}}{\beta_{1}}$ and $\chi[\mathbf{1 2}]_{\mathrm{SO}(12)}=\chi[\mathbf{6}, \mathbf{1}]+\chi[\mathbf{1}, \mathbf{6}]$. This shows that the index can indeed be written in characters of $\mathrm{U}(1) \times \mathrm{SU}(2) \times \mathrm{SO}(12)$, at least to the evaluated order.

Finally we consider gluing tubes $I$ and $I I I$. Due to the cycling of the global symmetry necessary when connecting the two tubes, we again need to shift the fluxes for tube III. Summing the two fluxes, we associate with the resulting tube the flux $\left(\frac{1}{2}, \frac{1}{4}, \frac{1}{4}, 0\right)+$ $\left(\frac{1}{2}, 0, \frac{1}{4},-\frac{1}{4}\right)=\left(1, \frac{1}{4}, \frac{1}{2},-\frac{1}{4}\right)$.

We can next close the tube to a torus. When doing this we are forced to identify $\beta_{2}=\beta_{3}=\frac{1}{\gamma_{2}}$. The resulting theory is shown in figure 31 . Due to the required identification, we associate with this theory the flux $\left(1, \frac{1}{3}, \frac{1}{3}, \frac{1}{3}\right)$. We can test this by comparing anomalies where we indeed find they match the $6 d$ expectations. 


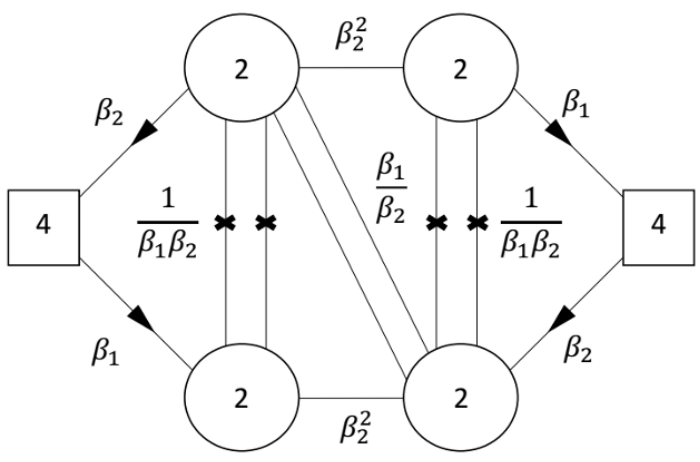

Figure 31. The theory resulting from gluing the tubes $I$ and $I I I$ together.

Duality with USp $(2 k) / \mathbf{S U}(\boldsymbol{k}+\mathbf{1})$ quivers. The construction of minimal type $D$ conformal matter can also be approached from a different perspective, and comparing the two then leads to interesting physical phenomena. Particularly, the minimal type $D$ conformal matter has, besides the $\mathrm{SU}(2)$ quiver description, two additional $5 d$ gauge theory descriptions, as a $\mathrm{USp}(2 k)$ and an $\mathrm{SU}(k+1)$ gauge theories with fundamental hypermultiplets. These can also be used to construct $4 d$ theories in a similar manner to that which is done here, but by using a $5 d$ domain wall extrapolating between the USp $(2 k)$ and the $\mathrm{SU}(k+1)$ descriptions. This construction was covered extensively in [11].

A rather interesting aspect in this comparison is that we can construct the same compactification using different tubes. This should then give two dual descriptions of the same theory, that has at its heart the duality between the different $5 d$ gauge theory descriptions of the minimal type $D$ conformal matter. The simplest case here is to use the tube with all plus boundary condition by gluing a multiple of $k+1$ of them to form a torus. When $k$ is odd then this compactification can be easily built from the tubes introduced in [11]. This leads to a duality between a plane quiver theory of $\mathrm{SU}(2)$ gauge groups and a circular quiver of alternating $\mathrm{USp}(2 k)$ and $\mathrm{SU}(k+1)$ groups. This case was discussed in appendix of [11].

From the constructions presented both here and in [11] we can build a large number of different examples as in both cases we have ample tools to engineer torus compactifications with different values of flux. For instance we considered an example for $D_{5}$ involving the two tubes called $I I$ and $I I I$. From these we can engineer a theory with flux $(1,-1,1,-1,0,0,0,0,0,0)$, similarly to how we constructed the theory in figure 30 .

We can also, using the tubes associated with the USp/SU construction, build a torus compactification with the same flux. In fact, we can construct the torus compactification associated with flux $\left(\frac{1}{2}, \frac{1}{2}, \frac{1}{2}, \frac{1}{2}, 0,0,0,0,0,0\right)$, which naively should be dual to the theory in figure 30, as the fluxes are the same up to a Weyl transformation. However, as previously stated, this flux requires also a central element in the global symmetry to be consistently quantized, and the resulting theories differ in these central elements. Particularly, for correct quantization a $\mathbb{Z}_{2}$ central flux is required. For the theory in figure 30, this central flux is embedded in the center of the SU(4) global symmetry. However, for the analogous theory in the USp/SU construction, this central flux is embedded in the center of the $\mathrm{SO}(12)$ global symmetry. 


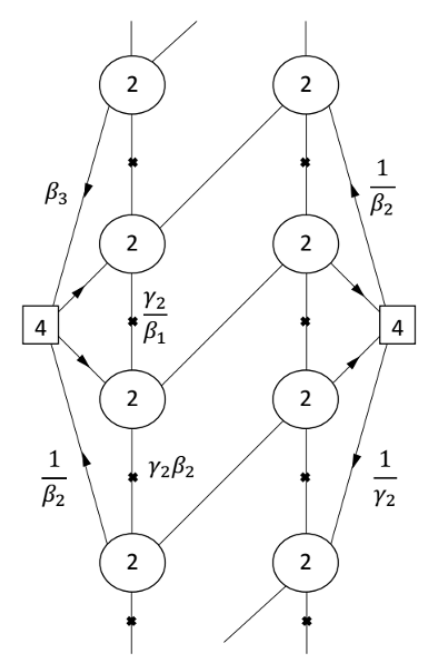

(a)

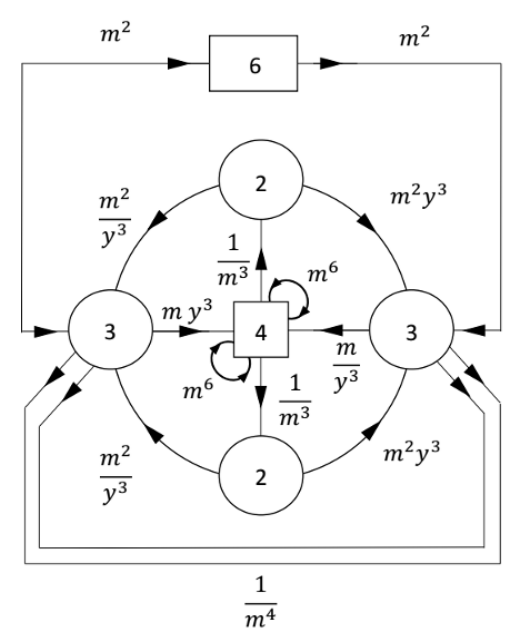

(b)

Figure 32. Two theories expected to be dual since they both describe the same compactification of the minimal $\left(D_{5}, D_{5}\right)$ conformal matter. (a) The theory constructed from the tubes $I I$ and $I I I$ that we introduced previously. We have written some of the charges of the fields via fugacities, the rest can be inferred from anomalies and the superpotentials. (b) The theory constructed from $\mathrm{USp}(2 k) / \mathrm{SU}(k+1)$ domain wall discussed in [11]. Here we used the simpler version given after Intriligator-Pouliot duality. We refer the reader to [11] for the details. The gauge groups are $\mathrm{SU}(3)$ and $\mathrm{SU}(2)$ as it is the same as $\mathrm{USp}(2)$. The circular double arrows connected to the central $\mathrm{SU}(4)$ global symmetry group stand for chiral fields in the antisymmetric representation of said $\mathrm{SU}(4)$ flavor symmetry group. These flip the gauge invariant states made from the $\mathrm{SU}(2) \times \mathrm{SU}(4)$ bifundamentals.

As a result to get a duality we need to form a torus compactification with integer flux, the simplest case being $(1,-1,1,-1,0,0,0,0,0,0)$. In figure 32 we have drawn the two dual theories associated with this flux. Both are expected to have a $\mathrm{U}(1) \times \mathrm{SU}(4) \times \mathrm{SO}(12)$ global symmetry, which is the symmetry preserved by the flux. For the theory in figure 32 (b), $\mathrm{U}(1)_{m} \times \mathrm{SU}(4)$ should map to the first part, while $\mathrm{U}(1)_{y} \times \mathrm{SU}(6)$ should enhance to $\mathrm{SO}(12)$ as $\mathbf{1 2}_{\mathrm{SO}(12)}=y^{3} \mathbf{6}_{\mathrm{SU}(6)}+\frac{1}{y^{3}} \overline{\mathbf{6}}_{\mathrm{SU}(6)}$. For the theory in figure 32 (a), the combination $\mathrm{U}(1)_{\beta_{1}}+\mathrm{U}(1)_{\beta_{3}}-\mathrm{U}(1)_{\beta_{2}}-\mathrm{U}(1)_{\gamma_{2}}$ should map to the $\mathrm{U}(1)$, the other 3 combinations should build the $\mathrm{SU}(4)$ as $\mathbf{4}_{\mathrm{SU}(4)}=\sqrt{\frac{\beta_{1} \gamma_{2}}{\beta_{2} \beta_{3}}}\left(\beta_{1} \beta_{2}+\frac{1}{\beta_{1} \beta_{2}}\right)+\sqrt{\frac{\beta_{2} \beta_{3}}{\beta_{1} \gamma_{2}}}\left(\beta_{3} \gamma_{2}+\frac{1}{\beta_{3} \gamma_{2}}\right)$, and the two $\mathrm{SU}(4)$ groups should build $\mathrm{SO}(12)$ as $\mathbf{1 2}_{\mathrm{SO}(12)}=\mathbf{6}_{\mathrm{SU}(4)_{1}}+\mathbf{6}_{\mathrm{SU}(4)_{2}}$. The exact relation between the $\mathrm{U}(1)$ groups on both sides is expected to be: $\mathrm{U}(1)_{m}=\frac{3}{2}\left(\mathrm{U}(1)_{\beta_{1}}+\mathrm{U}(1)_{\beta_{3}}-\mathrm{U}(1)_{\beta_{2}}-\mathrm{U}(1)_{\gamma_{2}}\right)$, or in fugacities: $m^{6}=\frac{\beta_{1} \beta_{3}}{\beta_{2} \gamma_{2}}$.

Note that the theory one constructs using the USp/SU domain wall has $\mathrm{SU}(3)$ nodes with ten flavors. This is IR free theory and the way to understand the model is by first not gauging the SU(3) groups but only the USp(2) groups, see [11] for a discussion in closely related case. Let us first discuss the theory with only the $\mathrm{SU}(2)$ groups gauged and without the fields charged under $\mathrm{SU}(6)$ and $\mathrm{SU}(3)$. This theory has the symmetries $\mathrm{U}(1)_{m}$ and $\mathrm{U}(1)_{y}$ with only the former mixing with the R-symmetry. The model is asymptotically free and after a maximization we obtain that the superconformal R-symmetry is $R_{0}-0.00345 q_{m}$, 
where $R_{0}$ assigns R-charge $3 / 5$ to fields charged under the $\mathrm{SU}(2)$ with the rest fixed by the superpotential, with no unitarity bound violating operators. It is then plausible that the theory flows to an interacting conformal fixed point. We now add the six fundamental fields for both SU(3) flavor groups. Then we obtain that $\operatorname{Tr}\left(\operatorname{RSU}(3)^{2}\right)=6\left(\frac{2}{3}-1\right) \frac{1}{2}+\left(-3+2 \sqrt{\frac{19}{51}}\right)$ with the second term coming from the fixed point. We note that this term +3 , which is the contribution to $\operatorname{Tr}\left(R \mathrm{SU}(3)^{2}\right)$ from the $\mathrm{SU}(3)$ gauge field, is positive meaning that the $\mathrm{SU}(3)$ group is asymptotically free at the fixed point with the addition of the six fundamental fields. We remind the reader that the beta function is proportional to $-\operatorname{Tr}\left(\mathrm{U}(1)_{R} \mathrm{SU}(N)^{2}\right)$ with $R$ being the superconformal symmetry of the fixed point. We then flow to a fixed point with all operators above the unitarity bound, and the superpotential involving fields charged under $\mathrm{SU}(6)$ and $\mathrm{SU}(3)$ is marginal. This implies that the theory makes sense as a sequence of flows starting from weakly couple UV theory.

We can test the duality in various ways. First we can compare anomalies, where we find the anomalies indeed match between the two theories, with the expected identification, and also match the $6 d$ prediction. We can also compute and compare the superconformal index. We indeed find that it matches between the two theories, at least to the order we evaluated it. We also observe that it forms characters of the expected $\mathrm{U}(1) \times \mathrm{SU}(4) \times \mathrm{SO}(12)$ global symmetry. Specifically, we find for the index:

$$
\begin{aligned}
I= & 1+2 \frac{\beta_{1} \beta_{3}}{\beta_{2} \gamma_{2}} \chi[\mathbf{6}]_{\mathrm{SU}(4)}(p q)^{\frac{1}{3}} \\
& +(p q)^{\frac{2}{3}}\left(\sqrt{\frac{\beta_{1} \beta_{3}}{\beta_{2} \gamma_{2}}} \chi[\mathbf{4}]_{\mathrm{SU}(4)} \chi[\mathbf{1 2}]_{\mathrm{SO}(12)}+\frac{\beta_{1}^{2} \beta_{3}^{2}}{\beta_{2}^{2} \gamma_{2}^{2}}\left(3 \chi\left[\mathbf{2 0}^{\prime}\right]_{\mathrm{SU}(4)}+\chi[\mathbf{1 5}]_{\mathrm{SU}(4)}+3\right)\right)+\ldots
\end{aligned}
$$

Here we have used the notation of figure 32 (a), the transformation to the notation of the other theory can be done using the relations given above. We have also used the R-symmetry $\mathrm{U}(1)_{R}^{6 d}-\frac{1}{3}\left(\mathrm{U}(1)_{\beta_{1}}+\mathrm{U}(1)_{\beta_{3}}-\mathrm{U}(1)_{\beta_{2}}-\mathrm{U}(1)_{\gamma_{2}}\right)$, which is close to the superconformal R-symmetry which is $\mathrm{U}(1)_{R}^{6 d}-\sqrt{\frac{11}{126}}\left(\mathrm{U}(1)_{\beta_{1}}+\mathrm{U}(1)_{\beta_{3}}-\mathrm{U}(1)_{\beta_{2}}-\mathrm{U}(1)_{\gamma_{2}}\right)$. There are no operators violating the unitary bound with respect to the superconformal R-symmetry.

Finally we note that the first two terms in the index are exactly as expected from the compactification of $6 d$ theories based on the reasoning of [33] (see also appendix $\mathrm{E}$ in [10]). The third term is just the self-product of the first term.

\subsection{Examples of $E$}

Let us now give some illustrative computations for the compactifications of $E$ conformal matter. As the gauge groups in the relevant quiver diagrams become of large dimensions even in the minimal case, there are very few computations one can perform explicitly. We will thus restrict to checking anomalies and verifying indices in limiting cases of the minimal conformal matter.

$\boldsymbol{E}_{6}$. A typical tube is depicted in figure 14. As discussed in previous sections, the flux associated to the tubes is such that gluing the tube to itself six times one obtains integer flux preserving the full symmetry of the theory unbroken by the flux, which in this case is 

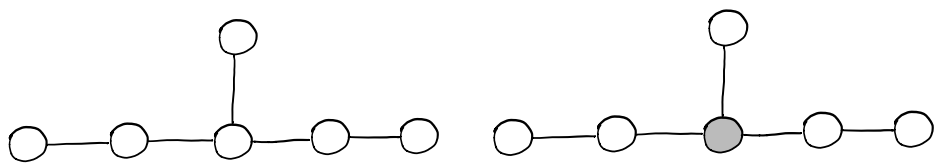

Figure 33. The Dynkin diagram of $E_{6} \times E_{6}$ with the shaded node corresponding to the node with the flux.

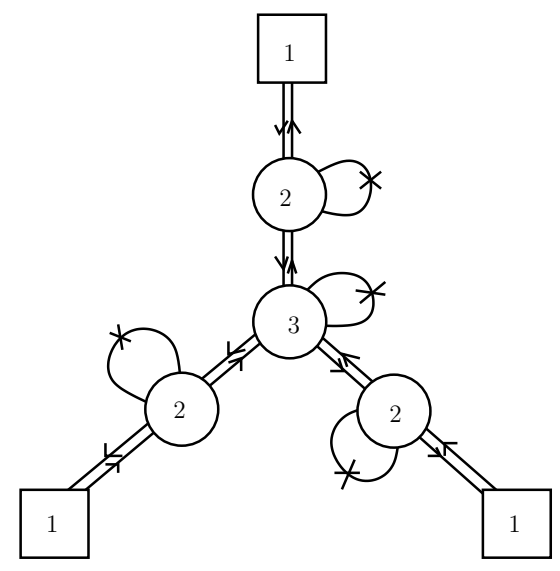

Figure 34. Torus with $1 / 6$ units of flux in the $U(1)$ corresponding to the central node. The lines from vertex to itself are adjoint plus a singlet.

$E_{6} \times \mathrm{SU}(3) \times \mathrm{SU}(3) \times \mathrm{SU}(2) \times \mathrm{U}(1)$. That is the flux of the combined model is in the $\mathrm{U}(1)$ corresponding to the central node of one of the $E_{6}$ groups. See figure 33 .

Gluing the tube to itself we obtain the affine quiver of figure 34. The figure is for the minimal case. For non minimal the groups become $\mathrm{SU}(l N)$ with the $l$ label appearing in the figure and all are gauge nodes. We will discuss only the minimal case in what follows.

Note that the flipping of the baryonic operator is irrelevant for the SU(3) gauge group. We can perform naive a maximization ignoring this and find that this agrees with the six dimensional computation. Computing the index we can see that protected states organize in $\mathrm{U}(1) \times E_{6}$ representations. The rest of the symmetry is broken by the fractional flux, where out of one of the two $E_{6}$ symmetries only the $\mathrm{U}(1)$ with the flux is not broken. Parametrizing the $\mathrm{U}(1)$ in the end of the legs of the quiver as $b_{i}$ and the $\mathrm{U}(1)$ under which the bifundamentals in the middle are charged by $a_{i}$, we also have an additional U(1) we denote by $t$ under which the bifundamental fields are charged half and the adjoint fields charged one. The simplest operators charged under $a$ and $b$ symmetries appear at $(q p)^{\frac{4}{3}}$ in the index computation and are in the following representations,

$$
(\mathbf{3}, \overline{\mathbf{3}}, \mathbf{1})+(\mathbf{3}, \mathbf{1}, \overline{\mathbf{3}})+(\overline{\mathbf{3}}, \mathbf{3}, \mathbf{1})+(\overline{\mathbf{3}}, \mathbf{1}, \mathbf{3})+(\mathbf{1}, \mathbf{3}, \overline{\mathbf{3}})+(\mathbf{1}, \overline{\mathbf{3}}, \mathbf{3}),
$$

where $\mathbf{3}_{l}=a_{l}^{2}+\frac{1}{a_{l}}\left(b_{l}^{-1}+b_{l}\right)$. The above representations naturally form $\mathbf{2 7} \oplus \overline{\mathbf{2 7}}$ of $E_{6}$, they have $\mathrm{U}(1)_{t}$ charge two. We can compute the index in a limit. Note that without the singlet fields this is an $\mathcal{N}=2$ model and it has an [34] HL limit. In terms of six dimensional $\mathrm{R}$ charge this corresponds to keeping $q p t$ fixed while sending $q, p, 1 / t$ to zero. Keeping the 


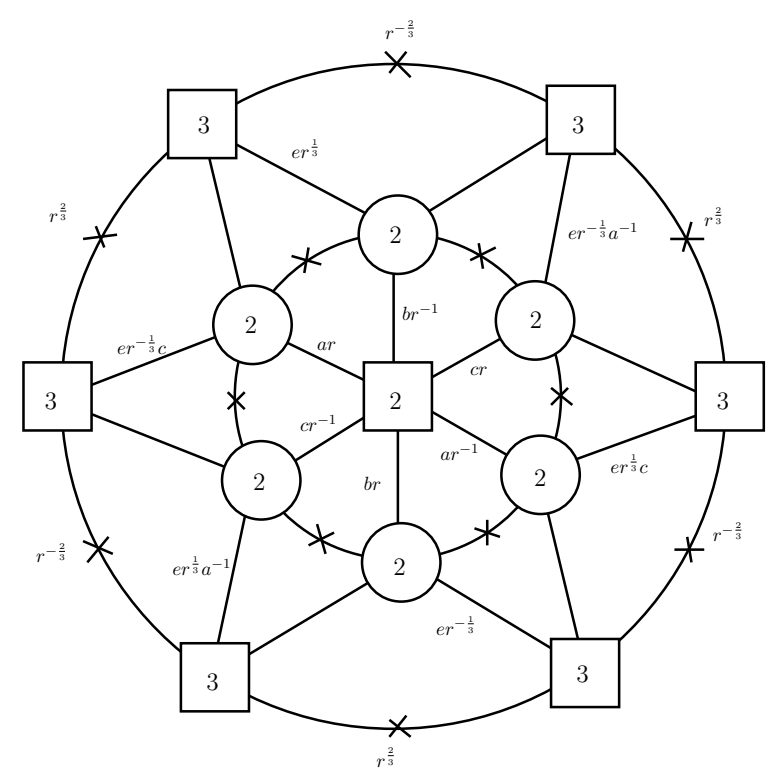

Figure 35. The leg of torus with one unit of flux. The links of the quiver without the flip fields are charges with charge half under $\mathrm{U}(1)_{t}$, the fields with the flip are charged minus one. We denoted the other charges on the quiver with the charges of links with no labels derivable from superpotentials. The $\mathrm{SU}(2)$ explicitly visible in the quiver is $\mathrm{SU}(2)_{f}$.

singlet fields in the bifundamentals does not spoil the limit but the flip fields give singular contributions. As the flip fields are free we can compute the index without these. We obtain,

$$
\begin{aligned}
1+2 t & +(3+\overline{\mathbf{2 7}}+\mathbf{2 7}) t^{2}+(-1+2 \mathbf{2 7}+2 \overline{\mathbf{2 7}}+\mathbf{7 8}) t^{3}+ \\
(-7 & \left.+\left(\operatorname{Sym}^{2}(3+\overline{\mathbf{2 7}}+\mathbf{2 7})\right)-3 \mathbf{7 8}-4 \overline{\mathbf{2 7}}-4 \mathbf{2 7}\right) t^{4}+\ldots
\end{aligned}
$$

Note that all the operators form $E_{6} \times \mathrm{U}(1)$ representations. We also mention that this model does not actually possess an $E_{6}$ symmetric point on its conformal manifold. This can be shown as the theory is conformal and all the exactly marginal deformations are $\mathcal{N}=2$. This is analogous to a similar statement in [10]. We do not have any contradictions for theories with higher amounts of flux having this symmetry.

We can combine the tubes to form integer value of flux. Gluing six two punctured spheres we obtain torus with flux one. We expect the full symmetry to be visible there. The theory can be composed of three copies of the one in figure 35 by gluing them along the perimeter. The anomaly conditions will identify

$$
\prod_{i=1}^{3} a_{i}=\prod_{j=1}^{3} c_{j}=\prod_{l=1}^{3} b_{l}=1
$$

with $\left(a_{n}, b_{n}, c_{n}\right)$ being the symmetries of the three copies, and $a_{n} c_{n} b_{n}=1$. The symmetry is then given by the three $\mathrm{SU}(2) \mathrm{s}$ of the three copies, three $\mathrm{U}(1)_{e_{n}}$, one $\mathrm{U}(1)_{r}$, one $\mathrm{U}(1)_{t}$, three copies of $\mathrm{U}(1)_{a} \mathrm{U}(1)_{b} \mathrm{U}(1)_{c}$ subject to five constraints. The rank of the symmetry is 
twelve as expected. The index in the limit above is given as follows,

$$
\begin{aligned}
1+ & (\mathbf{2}, \mathbf{3}, \mathbf{3} ; \mathbf{1}) t+(-(\mathbf{1}+\mathbf{3}, \overline{\mathbf{3}}, \overline{\mathbf{3}} ; \mathbf{1})-12+(\mathbf{1}+\mathbf{3}, \mathbf{6}, \overline{\mathbf{3}} ; \mathbf{1})+(\mathbf{1}+\mathbf{3}, \overline{\mathbf{3}}, \mathbf{6} ; \mathbf{1})) t^{2}+ \\
& +2(\mathbf{1}, \mathbf{3}, \mathbf{1} ; \mathbf{2 7}) t^{2}+2(\mathbf{1}, \mathbf{1}, \mathbf{3} ; \overline{\mathbf{2 7}}) t^{2}+\ldots
\end{aligned}
$$

Here we have the representations of $\left(\mathrm{SU}(2)_{r}, \mathrm{SU}(3)_{\alpha}, \mathrm{SU}(3)_{\gamma} ; E_{6}\right)$. The characters are,

$$
\begin{aligned}
& \mathbf{2}_{\mathrm{SU}(2)_{r}}=\frac{1}{r^{2}}+r^{2}, \quad \mathbf{2 7}_{E_{6}}=\left(\overline{\mathbf{3}}_{1}, \mathbf{3}_{2}, \mathbf{1}_{3}\right)+\left(\mathbf{1}_{1}, \overline{\mathbf{3}}_{2}, \mathbf{3}_{3}\right)+\left(\mathbf{3}_{1}, \mathbf{1}_{2}, \overline{\mathbf{3}}_{3}\right), \\
& \mathbf{3}_{i}=e_{i}^{\prime-2}+e_{i}^{\prime}\left(\frac{1}{f_{i}}+f_{i}\right), \quad e_{i}=e_{i}^{\prime}\left(a_{i}^{2} b_{i}\right)^{\frac{1}{3}}, \quad \mathbf{3}_{\alpha}=\alpha_{1}+\alpha_{2}+\frac{1}{\alpha_{2} \alpha_{1}}, \quad \mathbf{3}_{\gamma}=\gamma_{2}+\gamma_{1}+\frac{1}{\gamma_{2} \gamma_{1}}, \\
& a_{1}=\left(\gamma_{2} \gamma_{1} \alpha_{1} \alpha_{2}\right)^{-\frac{1}{2}}, \quad b_{1}=\left(\alpha_{2} \gamma_{2}\right)^{\frac{1}{2}}, \quad b_{2}=\left(\gamma_{1} / \alpha_{1} \alpha_{2}\right)^{\frac{1}{2}}, \quad a_{2}=\left(\alpha_{1} \gamma_{2}\right)^{\frac{1}{2}} \text {. }
\end{aligned}
$$

One can actually understand some of the terms in the index from six dimensions. Note that under $\mathrm{U}(1)_{t}$ which has the flux we have,

$$
\mathbf{7 8}=\mathbf{3}_{\alpha} \mathbf{3}_{\gamma}\left(t^{-2}+t \mathbf{2}_{r}\right)+\overline{\mathbf{3}}_{\alpha} \overline{\mathbf{3}}_{\gamma}\left(t^{2}+t^{-1} \mathbf{2}_{r}\right)+\mathbf{2}_{r}\left(t^{-3}+t^{3}\right)+\mathbf{3}_{r}+\mathbf{8}_{\gamma}+\mathbf{8}_{\alpha}+1 .
$$

We expect this term to contribute at order $q p$ with the six dimensional R-symmetry with the multiplicities determined by flux and charges under $t$ (see [10] appendix E for this statement which summarizes the results of [33]). In our limit we see that the term $\mathbf{3}_{\alpha} \mathbf{3}_{\gamma} \mathbf{2}_{r}$ should survive and contribute to the index at order $t$, which we observe. The states with $\overline{\mathbf{3}}_{\gamma} \overline{\mathbf{3}}_{\alpha} t^{2}$ should contribute with multiplicity 2 . Note that these are divergent in the limit we take and they are captured exactly by the flip fields in the smaller circles of the three legs of the quiver. The states with $t^{3} \mathbf{2}_{r}$ contribute with multiplicity three and also are divergent and they are captured by flip fields coming from the large circle in the center of the quiver. The remaining states in the adjoint of $E_{6}$ vanish in the limit. The states we see in representations of the $E_{6}$ which is invariant under the flux is also easy to understand. The six dimensional theory has operators in $\mathbf{2} \mathbf{7}_{a} \otimes \overline{\mathbf{2}} \mathbf{7}_{b} \oplus \overline{\mathbf{2}} \mathbf{7}_{a} \otimes \mathbf{2} \mathbf{7}_{b}$ at R-charge four. The operators which survive the limit have charge two under $\mathrm{U}(1)_{t}$ and thus appear with factor of two precisely as the representations appearing in the index.

Let us compute for completeness the flux of a tube following from our assignment of symmetries. Note that we have two factors of $E_{6}$ which we will denote as $\beta$ and $\gamma$. The flux is only in one of them. Reading off the charges of the flip fields appearing in the quiver of figure 35 we obtain that the only non vanishing charge is in $t$ and is equal to 144 . We need to add also the contribution of the flip fields flipping the bifundamenta baryons between pairs of SU(1) groups. Although in this case the baryons are just the fields and the flipping removes them, the prescription of counting the charges is to take all the flip fields. We also note that the symmetries satisfy (4.18). This theory is built from six tubes so we have to divide by six to obtain flux of a single tube and then further divide by 24 which sets the coefficient $n_{t}=\frac{3}{2}$ in (3.11). To obtain the flux for a single tube we need to read off the charges of a wedge in the figure.

One can consider other choices of the flux. For example taking the flux to be such that one of the $E_{6}$ factors is broken to $\mathrm{U}(1) \times \mathrm{SU}(6)$ we claim the torus with unit flux is in figure 36 . The node of the Dynkin diagram with the flux is depicted in figure 37 . 


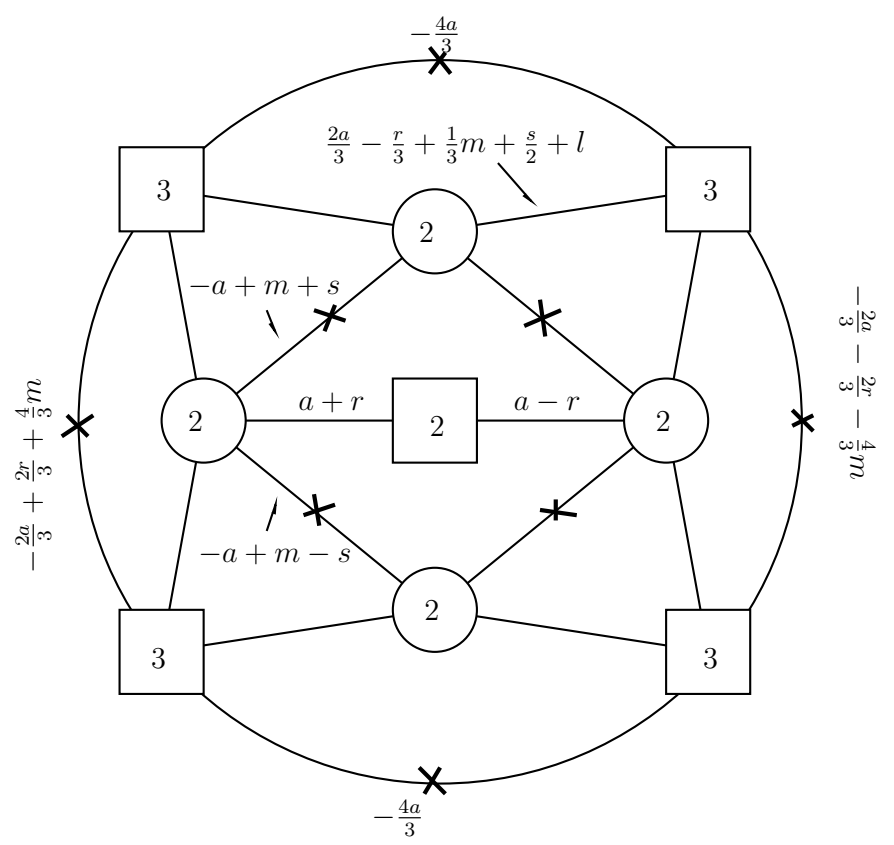

Figure 36. Leg of torus theory with one unit of flux breaking symmetry to $\mathrm{SU}(6) \mathrm{U}(1) E_{6}$. Three copies of this model are glued to by gauging the diagonal SU(3) symmetries. The charges under different symmetries are deduced from the superpotentials associated to the faces. The flux is for the $\mathrm{U}(1)_{a}$ symmetry. The $\mathrm{SU}(2)$ flavor symmetry with $\mathrm{U}(1)_{l}$ symmetry enhances to $\mathrm{SU}(3)$ for each leg and the three $\mathrm{SU}(3) \mathrm{s}$ from the three legs enhance to $E_{6}$.
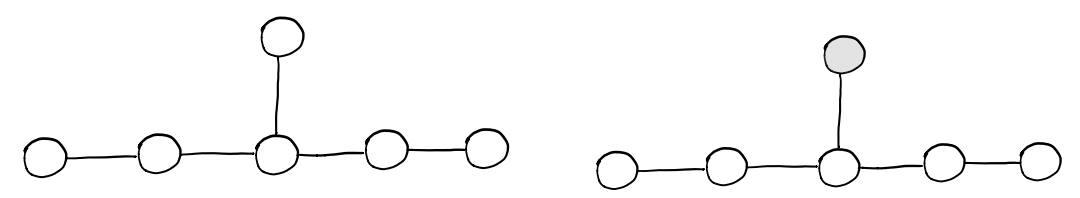

Figure 37. The Dynkin diagram of $E_{6} \times E_{6}$ with the shaded node corresponding to node with flux.

We can decompose the torus to two equal tubes such that each has fractional flux. We can also further decompose the tubes to two different ones having different flux. One can check that the anomalies of the model agree with six dimensions and that the index forms the representations of the symmetry, at least in similar limits as the one we discussed here.

$\boldsymbol{E}_{\mathbf{7}}$ and $\boldsymbol{E}_{\mathbf{8}}$. The basic tube here has the form of the affine Dynkin diagram of the $E_{7}$ and $E_{8}$ group. The gauge structure here is more involved than in other cases so explicit checks of the claims are harder to perform. Thus in this section we will restrict to discussing the affine quivers which are obtained by combining a single tube to form a torus. The flux in both cases is to the $\mathrm{U}(1)$ corresponding to the central node of one of the groups. The value of the flux is $\frac{1}{12}$ for the $E_{7}$ case and $\frac{1}{30}$ for the $E_{8}$ case. See figures 38 and 39 .

A theory, preserving all the symmetry, can be constructed when we combine multiples of the basic theory to get non fractional flux. The symmetry in the $E_{7}$ case is $\mathrm{SU}(4) \times$ $\mathrm{SU}(3) \times \mathrm{SU}(2) \times \mathrm{U}(1) \times E_{7}$ and is $\mathrm{SU}(5) \times \mathrm{SU}(2) \times \mathrm{SU}(3) \times E_{8} \times \mathrm{U}(1)$ for the case of $E_{8}$ (minimal) conformal matter. 


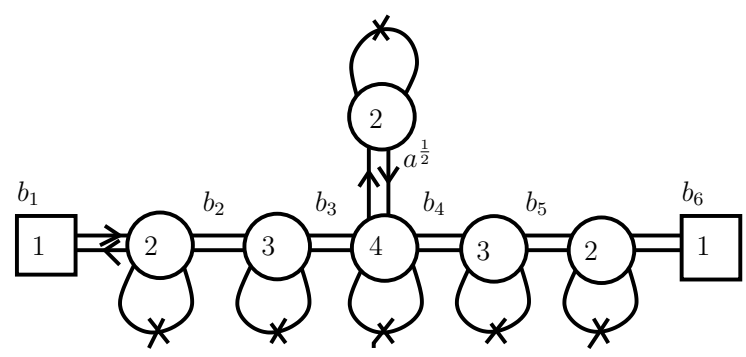

Figure 38. The quiver for the compactification of minimal $E_{7}$ conformal matter on torus with flux $1 / 12$ to the $\mathrm{U}(1)$ corresponding to the central node of one of the two $E_{7}$ symmetry factors. The fugacities denote the $\mathrm{U}(1)$ symmetries under which the bifundamental fields are charged. As usual all adjoints are charged under an additional $\mathrm{U}(1)$ with charge minus one and the bifundamentals are charged $1 / 2$.

We can compute the index in the limit we have studied. Let us quote the result for $E_{7}$. Without the flip fields, but with the additional singlets with same charges as the adjoints, this is given by,

$$
1+t+t^{2}+(\mathbf{5 6}+1) t^{3}+(\mathbf{1 3 3}+\mathbf{5 6}-5) t^{4}+\ldots
$$

We see that the index forms representations of $E_{7}$. We also see that the first $E_{7}$ representation is the $\mathbf{5 6}$ which enters at order $t^{3}$. This is in accordance with our discussion in section 2, where we noted that these classes of theories have operators in the bifundamental representation, which for the case of $E_{7}$ means one in the $(\mathbf{5 6}, \mathbf{5 6})$. Furthermore, in the minimal case considered here it is expected to contribute with R-charge 6 under the U(1) R-symmetry inherited from $6 d$. When converted to the index limit used here, this indeed gives an operator contributing at order $t^{3}$.

The way the $E_{7}$ representations arise is as follows. We decompose $E_{7}$ to $\mathrm{SO}(12) \times$ $\mathrm{SU}(2)$. The $\mathrm{SU}(2)$ Cartan is the $\mathrm{U}(1)_{a}$ symmerty appearing in figure 38 . The other $\mathrm{U}(1)$ symmetries map to the Cartan of $\mathrm{SO}(12)$. We denote $v_{i}$ to be Cartan of $\mathrm{SO}(12)$ so that the vector is

$$
\sum_{c=1}^{6} v_{c}^{ \pm 1} .
$$

Then the map of charges is,

$$
\begin{array}{rlrl}
b_{2} & =\left(v_{4} v_{1}^{2} v_{6}\right)^{\frac{1}{6}}, & b_{5}=\left(\frac{v_{2}}{v_{5} v_{3}^{2}}\right)^{\frac{1}{6}}, & b_{4}=\left(\frac{v_{2} v_{3}^{2}}{v_{5}}\right)^{\frac{1}{6}}, \\
b_{3}=\left(\frac{v_{4} v_{6}}{v_{1}^{2}}\right)^{\frac{1}{6}}, & b_{6}=\sqrt{v_{2} v_{5}}, & b_{1}=\sqrt{\frac{v_{4}}{v_{6}}}
\end{array}
$$

In general we would then make this assignment to the $\gamma$ copy of $E_{7}$ to the $M_{i}$ operators of the tube and then derive the charges with respect to $\beta$ copies according to the boundary conditions. 


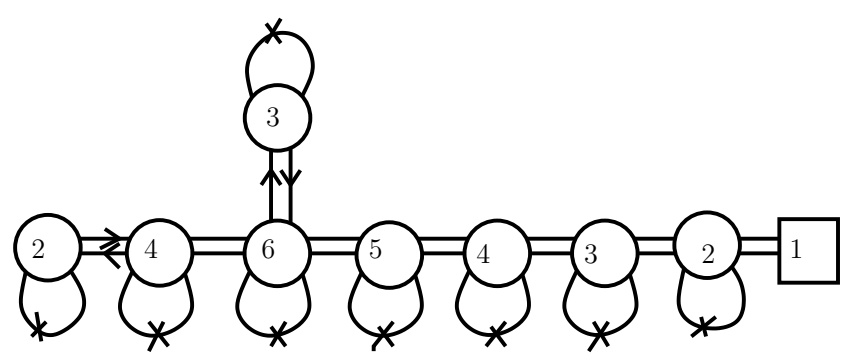

Figure 39. The affine quiver diagram corresponding to compactification on torus of $E_{8}$ minimal conformal matter with flux $1 / 30$ for the $\mathrm{U}(1)$ corresponding to central node of one of the two $E_{8}$ factors.

\section{Acknowledgments}

We like to thank SCGP summer workshop 2017 for hospitality during part of this work. The research of HK and CV is supported in part by NSF grant PHY-1067976. HK is supported in part by the National Research Foundation of Korea (NRF) Grant 2018R1D1A1B07042934. GZ is supported in part by World Premier International Research Center Initiative (WPI), MEXT, Japan. The research of SSR was supported by Israel Science Foundation under grant no. 1696/15 and by I-CORE Program of the Planning and Budgeting Committee.

Open Access. This article is distributed under the terms of the Creative Commons Attribution License (CC-BY 4.0), which permits any use, distribution and reproduction in any medium, provided the original author(s) and source are credited.

\section{References}

[1] J.J. Heckman, D.R. Morrison, T. Rudelius and C. Vafa, Atomic Classification of $6 D$ SCFTs, Fortsch. Phys. 63 (2015) 468 [arXiv:1502.05405] [INSPIRE].

[2] M. Del Zotto, J.J. Heckman, A. Tomasiello and C. Vafa, 6d Conformal Matter, JHEP 02 (2015) 054 [arXiv: 1407.6359] [inSPIRE].

[3] L. Bhardwaj, Classification of $6 d \mathcal{N}=(1,0)$ gauge theories, JHEP 11 (2015) 002 [arXiv: 1502.06594] [INSPIRE].

[4] D. Gaiotto, $\mathcal{N}=2$ dualities, JHEP 08 (2012) 034 [arXiv:0904.2715] [INSPIRE].

[5] F. Benini, Y. Tachikawa and B. Wecht, Sicilian gauge theories and $N=1$ dualities, JHEP 01 (2010) 088 [arXiv: 0909.1327] [inSPIRE].

[6] I. Bah, C. Beem, N. Bobev and B. Wecht, Four-Dimensional SCFTs from M5-Branes, JHEP 06 (2012) 005 [arXiv: 1203.0303] [INSPIRE].

[7] D. Gaiotto and S.S. Razamat, $\mathcal{N}=1$ theories of class $\mathcal{S}_{k}$, JHEP 07 (2015) 073 [arXiv: 1503.05159] [INSPIRE].

[8] S.S. Razamat, C. Vafa and G. Zafrir, $4 d \mathcal{N}=1$ from 6d (1,0), JHEP 04 (2017) 064 [arXiv: 1610.09178] [INSPIRE]. 
[9] I. Bah, A. Hanany, K. Maruyoshi, S.S. Razamat, Y. Tachikawa and G. Zafrir, $4 d \mathcal{N}=1$ from $6 d \mathcal{N}=(1,0)$ on a torus with fluxes, JHEP 06 (2017) 022 [arXiv: 1702.04740] [INSPIRE].

[10] H.-C. Kim, S.S. Razamat, C. Vafa and G. Zafrir, E-String Theory on Riemann Surfaces, Fortsch. Phys. 66 (2018) 1700074 [arXiv:1709.02496] [InSPIRE].

[11] H.-C. Kim, S.S. Razamat, C. Vafa and G. Zafrir, D-type Conformal Matter and SU/USp Quivers, JHEP 06 (2018) 058 [arXiv:1802.00620] [INSPIRE].

[12] K. Ohmori, H. Shimizu, Y. Tachikawa and K. Yonekura, $6 d \mathcal{N}=(1,0)$ theories on $T^{2}$ and class S theories: Part I, JHEP 07 (2015) 014 [arXiv: 1503.06217] [INSPIRE].

[13] K. Ohmori, H. Shimizu, Y. Tachikawa and K. Yonekura, $6 d \mathcal{N}=(1,0)$ theories on $S^{1} / T^{2}$ and class $S$ theories: part II, JHEP 12 (2015) 131 [arXiv:1508.00915] [INSPIRE].

[14] M. Del Zotto, C. Vafa and D. Xie, Geometric engineering, mirror symmetry and $6 \mathrm{~d}_{(1,0)} \rightarrow 4 \mathrm{~d}_{(\mathcal{N}=2)}$, JHEP 11 (2015) 123 [arXiv: 1504.08348] [INSPIRE].

[15] E. Nardoni, 4d SCFTs from negative-degree line bundles, JHEP 08 (2018) 199 [arXiv: 1611.01229] [INSPIRE].

[16] M. Fazzi and S. Giacomelli, $\mathcal{N}=1$ superconformal theories with $D_{N}$ blocks, Phys. Rev. D 95 (2017) 085010 [arXiv:1609.08156] [INSPIRE].

[17] A. Hanany and G. Zafrir, Discrete Gauging in Six Dimensions, JHEP 07 (2018) 168 [arXiv: 1804.08857] [INSPIRE].

[18] A. Hanany and N. Mekareeya, The small $E_{8}$ instanton and the Kraft Procesi transition, JHEP 07 (2018) 098 [arXiv: 1801.01129] [INSPIRE].

[19] K. Ohmori, H. Shimizu, Y. Tachikawa and K. Yonekura, Anomaly polynomial of general $6 d$ SCFTs, PTEP 2014 (2014) 103B07 [arXiv: 1408.5572] [INSPIRE].

[20] C.S. Chan, O.J. Ganor and M. Krogh, Chiral compactifications of 6-D conformal theories, Nucl. Phys. B 597 (2001) 228 [hep-th/0002097] [INSPIRE].

[21] D. Gaiotto and H.-C. Kim, Duality walls and defects in $5 d \mathcal{N}=1$ theories, JHEP $01(2017)$ 019 [arXiv: 1506.03871] [INSPIRE].

[22] P. Hořava and E. Witten, Eleven-dimensional supergravity on a manifold with boundary, Nucl. Phys. B 475 (1996) 94 [hep-th/9603142] [INSPIRE].

[23] P. Hořava and E. Witten, Heterotic and type-I string dynamics from eleven-dimensions, Nucl. Phys. B 460 (1996) 506 [hep-th/9510209] [INSPIRE].

[24] Y. Tachikawa, Instanton operators and symmetry enhancement in 5d supersymmetric gauge theories, PTEP 2015 (2015) 043B06 [arXiv:1501.01031] [INSPIRE].

[25] K. Yonekura, Instanton operators and symmetry enhancement in 5d supersymmetric quiver gauge theories, JHEP 07 (2015) 167 [arXiv: 1505.04743] [INSPIRE].

[26] C. Romelsberger, Counting chiral primaries in $N=1, d=4$ superconformal field theories, Nucl. Phys. B 747 (2006) 329 [hep-th/0510060] [INSPIRE].

[27] J. Kinney, J.M. Maldacena, S. Minwalla and S. Raju, An Index for 4 dimensional super conformal theories, Commun. Math. Phys. 275 (2007) 209 [hep-th/0510251] [INSPIRE].

[28] C. Beem and A. Gadde, The $\mathcal{N}=1$ superconformal index for class $S$ fixed points, JHEP 04 (2014) 036 [arXiv:1212.1467] [INSPIRE]. 
[29] F.A. Dolan and H. Osborn, Applications of the Superconformal Index for Protected Operators and q-Hypergeometric Identities to $N=1$ Dual Theories, Nucl. Phys. B 818 (2009) 137 [arXiv: 0801.4947] [INSPIRE].

[30] A. Hanany and K. Maruyoshi, Chiral theories of class $\mathcal{S}$, JHEP 12 (2015) 080 [arXiv: 1505. 05053] [INSPIRE].

[31] S. Franco, H. Hayashi and A. Uranga, Charting Class $\mathcal{S}_{k}$ Territory, Phys. Rev. D 92 (2015) 045004 [arXiv: 1504.05988] [INSPIRE].

[32] N. Seiberg, Electric-magnetic duality in supersymmetric nonAbelian gauge theories, Nucl. Phys. B 435 (1995) 129 [hep-th/9411149] [INSPIRE].

[33] C. Beem, S.S. Razamat and G. Zafrir, to appear.

[34] A. Gadde, L. Rastelli, S.S. Razamat and W. Yan, Gauge Theories and Macdonald Polynomials, Commun. Math. Phys. 319 (2013) 147 [arXiv:1110.3740] [InSPIRE]. 